\section{Pacific Northwest}

National Laboratory

Operated by Battelle for the

U.S. Department of Energy

\title{
Selective Media for Actinide Collection and Pre-Concentration: Results of FY 2006 Studies
}

\author{
G. J. Lumetta \\ T. G. Levitskaia \\ R. S. Addleman \\ S. I. Sinkov \\ B. P. Hay \\ L. A. Snow \\ T. L. Hubler \\ M. G. Warner \\ Pacific Northwest National Laboratory \\ and \\ S. L. Latesky \\ The University of the Virgin Islands
}

November 2006

Prepared for the U.S. Department of Energy under Contract DE-AC05-76RL01830 


\title{
DISCLAIMER
}

This report was prepared as an account of work sponsored by an agency of the United States Government. Neither the United States Government nor any agency thereof, nor Battelle Memorial Institute, nor any of their employees, makes any warranty, express or implied, or assumes any legal liability or responsibility for the accuracy, completeness, or usefulness of any information, apparatus, product, or process disclosed, or represents that its use would not infringe privately owned rights. Reference herein to any specific commercial product, process, or service by trade name, trademark, manufacturer, or otherwise does not necessarily constitute or imply its endorsement, recommendation, or favoring by the United States Government or any agency thereof, or Battelle Memorial Institute. The views and opinions of authors expressed herein do not necessarily state or reflect those of the United States Government or any agency thereof.

\author{
PACIFIC NORTHWEST NATIONAL LABORATORY \\ operated by \\ BATTELLE \\ for the \\ UNITED STATES DEPARTMENT OF ENERGY \\ under Contract DE-ACO5-76RL01830
}

Printed in the United States of America
Available to DOE and DOE contractors from the
Office of Scientific and Technical Information,
P.O. Box 62, Oak Ridge, TN 37831-0062;
ph: (865) 576-8401
fax: (865) 5765728
email: reports@adonis.osti.gov

\footnotetext{
Available to the public from the National Technical Information Service, U.S. Department of Commerce, 5285 Port Royal Rd., Springfield, VA 22161 ph: (800) 553-6847 fax: (703) 605-6900

email: orders@nits.fedworld.gov online ordering: http://www.ntis.gov/ordering.htm
} 


\title{
Selective Media for Actinide Collection and Pre- Concentration: Results of FY 2006 Studies
}

\author{
G. J. Lumetta \\ T. G. Levitskaia \\ R. S. Addleman \\ S. I. Sinkov \\ B. P. Hay \\ L. A. Snow \\ T. L. Hubler \\ M. G. Warner \\ Pacific Northwest National Laboratory \\ and \\ S. L. Latesky \\ The University of the Virgin Islands
}

November 2006

Prepared for the U.S. Department of Energy

under Contract DE-AC05-76RL01830

Pacific Northwest National Laboratory

Richland, Washington 99352 


\section{Summary}

In this work, we have investigated new materials for potential use in automated radiochemical separations. The work can be divided into three primary tasks: 1) synthesis of new ligands with high affinity for actinide ions, 2) evaluation of new materials for actinide ion affinity, and 3) computational design of advanced ligand architectures for highly selective binding of actinide ions.

\section{Ligand Synthesis}

Work was conducted on synthesizing Kläui ligand derivatives containing functionalized pendant groups on the cyclopentadienyl ring. The functionalized pendent groups would allow these ligands to be attached to organic and inorganic solid supports. This work focused on synthesizing the compound $\mathrm{Na}\left[\mathrm{Cp}^{\prime} \mathrm{Co}\left\{\mathrm{PO}\left(\mathrm{OC}_{2} \mathrm{H}_{5}\right)_{2}\right\}_{3}\right]$, where $\mathrm{Cp}^{\prime}=\mathrm{C}_{5} \mathrm{H}_{4} \mathrm{C}(\mathrm{O}) \mathrm{OCH}_{3}$. Synthesizing this compound is feasible, but the method used in FY 2006 produced an impure material. A modified synthetic scheme has been developed and will be pursued in FY 2007.

Work was also initiated on synthesizing bicyclic diamides functionalized for binding to polymeric resins or other surfaces. Researchers at the University of Oregon are collaborators in this work. To date, this effort has focused on synthesizing and characterizing a symmetrically substituted bicyclic diamide ligand with the $-\mathrm{COOH}$ functionality. Again, this synthetic effort will continue into FY 2007.

\section{Separations Material Evaluation}

Work was conducted in FY 2006 to provide a more extensive set of data on the selectivity and affinity of extraction chromatography resins prepared by sorption of Kläui ligand onto an inert macroreticular polymeric support. Consistent with previous observations, it was found that these materials strongly bind tetravalent actinides. These materials also adsorb trivalent actinides at low nitric acid concentrations, but the affinity for the trivalent actinides decreases with increasing nitric acid concentration. These materials have relatively low affinity for U(VI), but they do sorb U(VI) to a greater extent than $\mathrm{Am}(\mathrm{III})$ at $\left[\mathrm{HNO}_{3}\right]>0.3 \mathrm{M}$. Preliminary results suggest that the Kläui resins can separate $\mathrm{Pu}(\mathrm{IV})$ from sample solutions containing high concentrations of competing ions. Conceptual protocols for recovery of the $\mathrm{Pu}$ from the resin for subsequent analysis have been proposed, but further work is needed to perfect these techniques. Work on this subject will be continued in FY 2007. Automated laboratory equipment (in conjunction with Task 3 of the NA-22 Automation Project) will be used in FY 2007 to improve the efficiency of these experiments.

The sorption of actinide ions on self-assembled monolayer on mesoporous supports materials containing diphosphonate groups was also investigated. These materials also showed a very high affinity for tetravalent actinides, and they also sorbed U(VI) fairly strongly.

\section{Computational Ligand Design}

An extended MM3 molecular mechanics model was developed for calculating the structures of Kläui ligand complexes. This laid the groundwork necessary to perform the computer-aided design of bis-Kläui architectures tailored for $\mathrm{Pu}(\mathrm{IV})$ complexation. Calculated structures of the Kläui ligand complexes 
$\left[\mathrm{Pu}(\mathrm{Kläui})_{2}\left(\mathrm{OH}_{2}\right)_{2}\right]^{2+}$ and $\left[\mathrm{Fe}(\mathrm{Kläui})_{2}\right]^{+}$indicate a "bent" sandwich arrangement of the Kläui ligands in the $\mathrm{Pu}$ (IV) complex, whereas the Fe(III) complex prefers a "linear" octahedral arrangement of the two Kläui ligands. This offers the possibility that two Kläui ligands can be tethered together to form a material with very high binding affinity for $\mathrm{Pu}(\mathrm{IV})$ over Fe(III). The next step in the design process is to use de novo molecule building software (HostDesigner) to identify potential candidate architectures. 


\section{Acronyms}

$\begin{array}{ll}\text { BMA } & \text { bicyclic malonamide } \\ \text { CSD } & \text { Cambridge Structure Database } \\ \text { EN } & \text { electronegativity } \\ \text { FTIR } & \text { Fourier transform infrared } \\ \text { IC } & \text { ionic character } \\ \text { IR } & \text { ionic radius } \\ \text { LSC } & \text { liquid scintillation counting } \\ \text { MM3 } & \text { molecular mechanics model } \\ \text { NIST } & \text { National Institute of Standards and Technology } \\ \text { PNNL } & \text { Pacific Northwest National Laboratory } \\ \text { SAMMS } & \text { self-assembled monolayer on mesoporous supports } \\ \text { SNM } & \text { special nuclear material } \\ \text { SRM } & \text { standard reference material }\end{array}$





\section{Acknowledgments}

This work was funded by the National Nuclear Security Agency (NA-22). Pacific Northwest National Laboratory is operated by Battelle for the U.S. Department of Energy under Contract DE-AC05-76RL01830. The authors thank Michelle Malone and Surria James for their technical assistance in the Kläui ligand synthesis and Brendan Twamley of the University of Idaho for performing the X-ray crystal structure determination. The authors also thank Wayne Cosby and Brian Rapko for their review of this manuscript. 



\section{Contents}

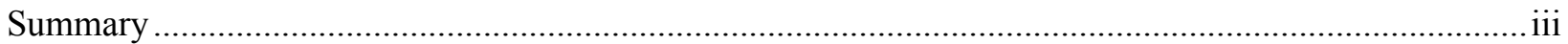

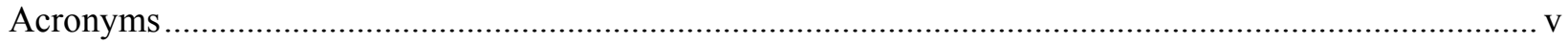

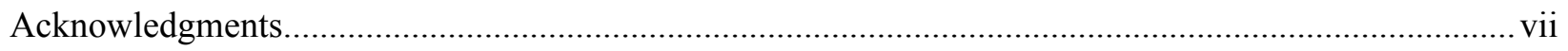

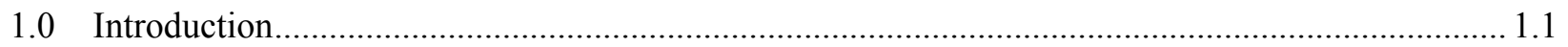

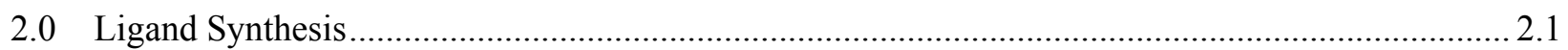

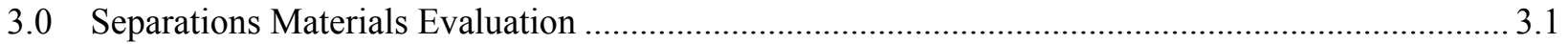

3.1 Batch Equilibrium Distribution Experiments with the nPr-Kläui Resin ............................... 3.1

3.2 Acid Dependence of Actinide(III, IV, V, and VI) and Eu(III) Sorption by the nPr-Kläui

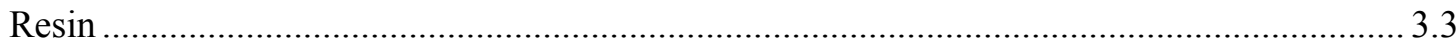

3.2.1 Effect of Initial Aqueous Pu(IV) Concentration on Pu(IV) Sorption by nPr-Kläui

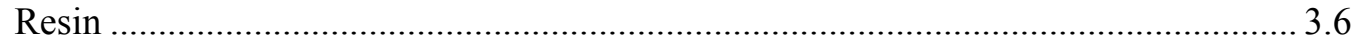

3.2.2 Selectivity and Effect of nPr-Kläui Resin Loading on Actinide (III, IV, V, and

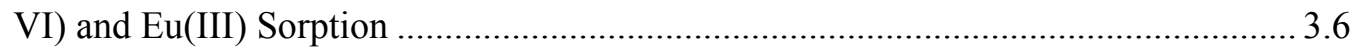

3.3 Attempted Elution of $\mathrm{Pu}(\mathrm{IV})$ from Kläui Resins by Reducing to $\mathrm{Pu}(\mathrm{III})$............................. 3.9

3.4 Elution of $\mathrm{Pu}(\mathrm{IV})$ from Kläui Resins by Removing the Ligand from the Resin Support...... 3.10

3.5 Batch Equilibrium Distribution Experiments with the SAMMS Materials .......................... 3.14

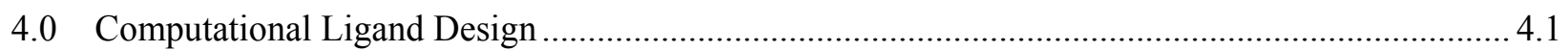

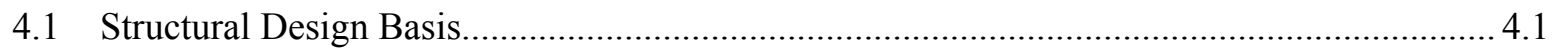

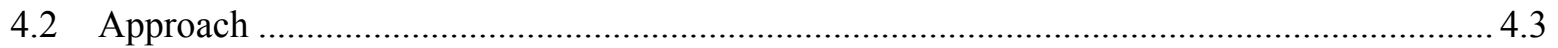

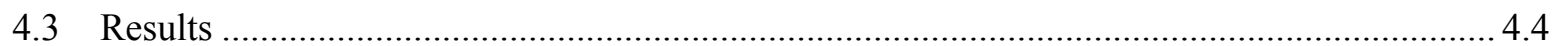

4.3.1 Analysis of Crystal Structure Data for Kläui Metal Complexes................................. 4.4

4.3.2 Extension of MM3 to Treat Kläui Ligands and their Metal Complexes....................... 4.8

4.3.3 Predicted Geometry for a $\left[\mathrm{Pu}(\mathrm{Kläui})_{2}\left(\mathrm{OH}_{2}\right)\right]^{2+}$ Complex ....................................... 4.21

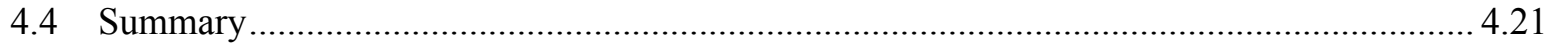

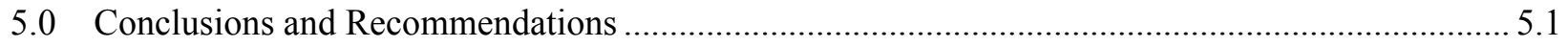

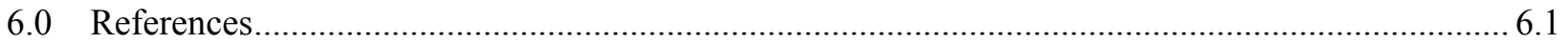




\section{Figures}

1.1. Bicyclic Diamide Ligand with High Affinity for Trivalent Lanthanide and Actinide Ions.

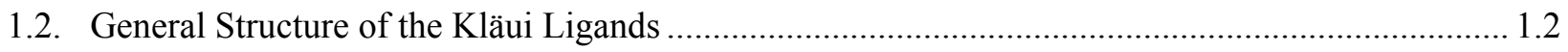

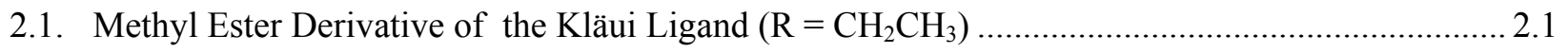

2.2. Synthetic Scheme for Functionalizing Kläui Ligands .......................................................... 2.2

2.3. ORTEP Plot of the Crystals Obtained from Crystallization of Product Containing Compound 2; Note the Reduction of the Ester Groups to Methyl Groups on the Cp Ring.................................... 2.2

2.4. Schematic Depiction of a Functionalized Kläui Ligand Covalently Bound to an Organic Resin .... 2.4

2.5. Structure of BMA Ligands Showing Symmetric and Asymmetric Substitution of the $-\mathrm{COOH}$

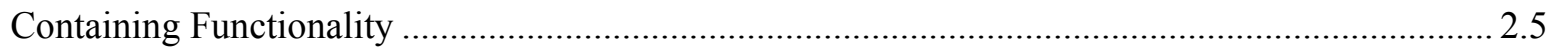

2.6. Illustration of the Functionality Present on the Surface of the PL-Oxime Resin.......................... 2.5

3.1. Acid Dependence of Actinide (III, IV, V, and VI) and Eu(III) Sorption by the ${ }^{\mathrm{n}} \mathrm{Pr}-\mathrm{Kläui}$ Resin

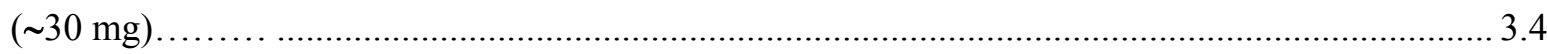

3.2. UV-vis Spectra of the ${ }^{n} \operatorname{Pr}-K l a ̈ u i$ Resin Equilibrated with $\mathrm{Np}(\mathrm{IV})$ (A) and $\mathrm{Np}(\mathrm{V})$ (B) Solutions

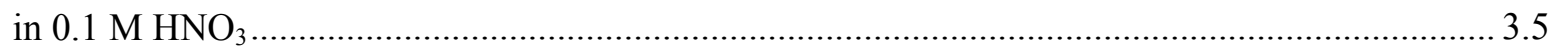

3.3. Acid Dependence of $\mathrm{Pu}(\mathrm{IV})$ Sorption at Variable ${ }^{\mathrm{n}} \mathrm{Pr}-\mathrm{Kläui} \mathrm{Resin} \mathrm{Loading} \mathrm{...................................} 3.7$

3.4. Effect of Initial Metal Ion Concentration (A) and ${ }^{\mathrm{n}}$ Pr-Kläui Resin Loading (B) on Actinide

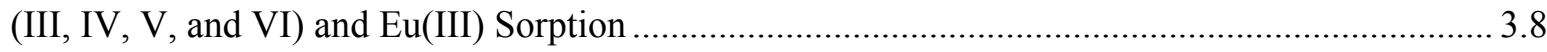

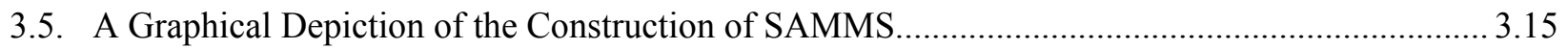

3.6. Batch Distribution Coefficients for Actinide Ions on Three Different SAMMS Materials............ 3.18

4.1. Illustration Showing How the Kläui Ligand Interacts with a Metal Ion....................................... 4.1

4.2. Crystal Structures of Six-Coordinate $\mathrm{Mn}^{3+}$ (left) and Eight-Coordinate $\mathrm{Eu}^{3+}$ (right) Complexes Containing Two Kläui Ligands................................................................................................ 4.2

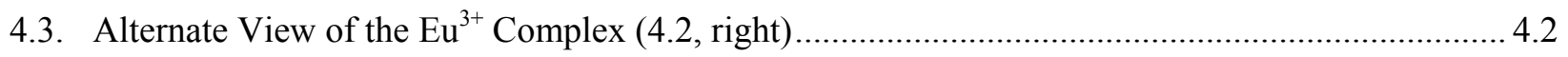

4.4. A Conceptual Hexadentate Ligand Formed by Linking Two Kläui Ligands to a Scaffold via Phosphonate Ester Bonds.

4.5. Examples of the Types of Kläui-Metal Complex Structures Available in the CSD ........................ 4.4

4.6. Histograms of Bond Distances Observed in Kläui Ligand Crystal Structures .............................. 4.6

4.7. Histograms of Angles Observed in Kläui Ligand Crystal Structures ........................................... 4.7 
4.8. Histograms of Dihedral Angles Observed in Kläui Ligand Crystal Structures

4.9. The Cyclopentadienyl Anion Ligand and its Complex with Co(III) Can Be Modeled with PCModel After Adding the Above Parameters to the Default MM3 Set

4.10. An Alkylphosphonate Molecule Can Be Modeled with PCModel After Adding the Above Parameters to the Default MM3 Set.

4.11. Phosphonate Co(III) Complexes Can Be Modeled with the PCModel After Adding the Above Parameters Once the Default Set Has Been Extended as Shown in 4.10

4.12. Views of the MM3 Optimized Kläui Ligand (left) and a Comparison of Calculated Versus Observed Structural Features (right).

4.13. Metal-Dependent Parameters for Modeling Kläui-Metal Complexes

4.14. Superpositions of X-ray Crystal Structure Geometries (colored) and Calculated Geometries (black) of Kläui-Metal Complexes 4.17

4.15. MM3 Optimized Geometries for $\left[\mathrm{Pu}(\mathrm{Kläui})_{2}\left(\mathrm{OH}_{2}\right)_{2}\right]^{2+}$ (left) and $\left[\mathrm{Fe}(\mathrm{Kläui})_{2}\right]^{+}$(right). 


\section{Tables}

3.1. Result of Ascorbic Acid and Hydroxylamine Nitrate Elution Experiments ................................ 3.10

3.2. Results of Experiment to Recover Pu from Et-Kläui Resin with Methanol ................................ 3.12

3.3. Results of Pu Ion Exchange Separation from Et-Kläui Residue ................................................ 3.13

3.4. Results of Experiment to Recover Pu from Dissolved Columbia River Sediment........................ 3.16

4.1. Mean Bond Distances $(\AA)$ and Angles $\left({ }^{\circ}\right)$ for the Kläui Ligand................................................. 4.5

4.2. Possible Interaction Types, Parameters Required to Describe them, and the Units Used in the

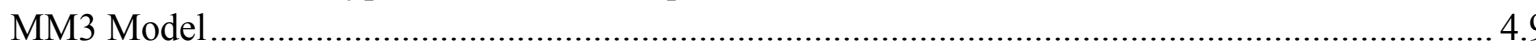

4.3. Physical Properties and Metal-Dependent Parameters for Selected Metal Ions .......................... 4.16 


\subsection{Introduction}

The work described in this report represents a subset of a larger effort to automate isotopic and radiochemical analyses. Two related but distinct applications exist:

- ultratrace analysis of actinide isotopes in environmental and other types of samples

- radiochemical analysis of radioactive samples for isotopic and elemental assay.

Chemical separation and analyte pre-concentration are integral to both of these applications. Current methods for separating and concentrating analytes for detection often involve complex wet chemical methods. The complexity of these separation schemes leads to relatively long sample preparation time, and automating such methods can be intricate. Sample preparation time and ease of automation can be greatly improved by using separation media that are highly specific for the analyte of interest.

Over the past decade, researchers at Pacific Northwest National Laboratory (PNNL) have developed a ligand design methodology using computational methods to guide experimental work in separations chemistry (Hay et al. 2004). The deliberate design of metal ion receptors requires the capability to distinguish hosts that efficiently complement the guest. Accurate electronic-structure calculations can be coupled with experimental data to provide fundamental knowledge about host structure and the nature of ion-binding site interactions. With a molecular-level understanding of how host architecture influences ion-binding affinity, it is possible to identify structures that will provide dramatic enhancements in ion binding affinity before they are prepared and tested. The power of this approach was demonstrated at PNNL recently after a deliberately designed bicyclic diamide structure (Figure 1.1) was found to extract $\mathrm{Eu}(\mathrm{III}) \sim 16$ million times more strongly than conventional malonamide structures (Lumetta et al. 2002, 2003).

Another class of actinide-selective ligands recently investigated at PNNL is the tripodal "Kläui ligands," which have the general chemical structure $\mathrm{Cp} * \mathrm{Co}\left[\mathrm{P}(\mathrm{O})(\mathrm{OR})_{2}\right]_{3}{ }^{-}\left[\mathrm{Cp}^{*}=\eta^{5}-\mathrm{C}_{5}\left(\mathrm{CH}_{3}\right)_{5}, \mathrm{R}=\mathrm{CH}_{3}\right.$, $\mathrm{C}_{2} \mathrm{H}_{5}$, etc.] (see Figure 1.2). We have observed high affinity of these ligands for the $\mathrm{Pu}(\mathrm{IV})$ ion. This presents an opportunity to apply these ligands to collecting and preconcentrating $\mathrm{Pu}(\mathrm{IV})$. In a step towards practical application of the Kläui ligands, we prepared a prototypical resin containing the Kläui ligand where $\mathrm{R}=\mathrm{C}_{2} \mathrm{H}_{5}$ (1b in Figure 1.2.). This resin contains $0.75 \mathrm{wt} \% 1 \mathrm{~b}$ sorbed onto macroreticular Amberlite ${ }^{\circledR}$ XAD-7 beads. Preliminary column experiments have indicated that this resin has remarkably high affinity for $\mathrm{Pu}(\mathrm{IV})$, even in the presence of large amounts of completing ions (e.g., $\mathrm{Al}^{3+}, \mathrm{Fe}^{3+}, \mathrm{Ca}^{2+}$, and $\mathrm{Li}^{+}$) (Lumetta et al. 2004). The $\mathrm{Pu}(\mathrm{IV})$ affinity is maintained over a large range of acid concentrations (e.g., $\mathrm{HCl}$ concentrations up to $9 \mathrm{M}$ ). Indeed, the resin binds $\mathrm{Pu}(\mathrm{IV})$ so strongly that it is difficult to elute from the resin, which is an issue discussed in this report. Similar behavior has been observed for an analogous resin containing $1 \mathrm{wt} \%$ ligand $1 \mathrm{c}$ on XAD-7.

Based on these observations, we believe that engineering the bicyclic diamide and the Kläui ligands into various separations media will allow for rapid collection and pre-concentration of $\mathrm{Pu}$ and other actinides for trace and ultra-trace detection. A number of engineered platforms will be considered, including attachment to organic resin beads and silica surfaces. Both of these options would allow for rapid and efficient concentrations of small amounts of analyte from a sample, which in turn will lower detection thresholds and decrease sample preparation time. 


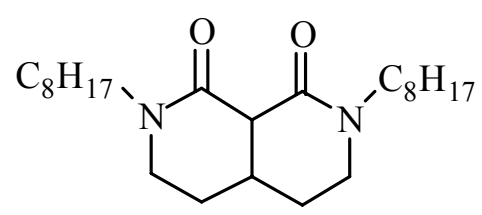

Figure 1.1. Bicyclic Diamide Ligand with High Affinity for Trivalent Lanthanide and Actinide Ions

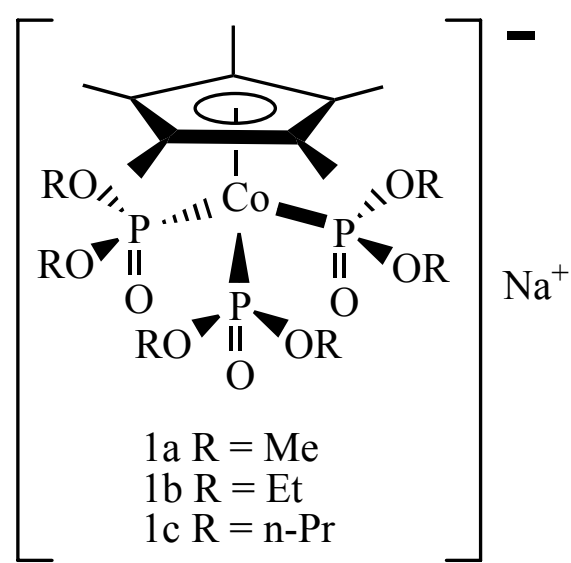

Figure 1.2. General Structure of the Kläui Ligands

This report summarizes work performed in FY 2006 on developing advanced separations media that can be used in automated radiochemical separations. The work has focused on three primary tasks:

1) synthesis of Kläui ligands and bicyclic diamide ligands that have pendant functional groups that allow chemical attachment to solid supports, 2) evaluation of separations media recently prepared at PNNL with potential application in automated actinide separations, and 3) computational design of advanced ligand architectures. The results of each of these tasks are discussed in the following individual sections. 


\subsection{Ligand Synthesis}

Small-scale chromatographic column separations are much more amenable to automation compared to other means of radiochemical separations, such as precipitation or solvent extraction. Incorporating bicyclic diamides or Kläui ligands into solid supports suitable for chromatographic applications would offer the potential to apply these ligands to radiochemical separations in automated systems. Towards this end, we undertook an effort to synthesize bicyclic diamides or Kläui ligands that contain functionalized pendent arms that can be covalently bound to organic or inorganic solid supports. Most of the synthetic effort in FY 2006 focused on preparing a Kläui ligand with a functionalized pendent group attached to the cyclopentadienyl $(\mathrm{Cp})$ ring of the ligand. Work was also initiated on preparing functionalized bicyclic diamide ligands, in collaboration with Prof. Jim Hutchison at the University of Oregon.

Attachment of a carbonyl functionality on the $\mathrm{Cp}$ ring of the Kläui ligand would provide a number of synthetic pathways to attach the ligand to various supports. For this reason, we set out to synthesize the ester derivative of the Kläui ligand (Figure 2.1). The synthetic approach to preparing this compound is summarized in Figure 2.2.

The functionalized cobaltocene (compound 1) was synthesized using a previously reported procedure (Hart et al. 1980). The material was isolated as a dark red solid after reaction of the functionalized $\mathrm{NaCp}^{\prime}\left(\mathrm{Cp}^{\prime}=\mathrm{C}_{5} \mathrm{H}_{4} \mathrm{CO}_{2} \mathrm{CH}_{3}^{-}\right)$ with anhydrous $\mathrm{CoBr}_{2}$ in tetrahydrofuran. The crude solid was triturated with pentane to obtain the solid product. The Fourier transform infrared (FTIR) spectrum of 1 indicated the presence of a strong $v(\mathrm{C}=\mathrm{O})$ band at $1696 \mathrm{~cm}^{-1}$.

Compound 2 was prepared using a modification of a previously reported procedure (Kläui et al. 1977). Refluxing compound 1 in neat diethylphosphite for $24 \mathrm{~h}$ caused a change

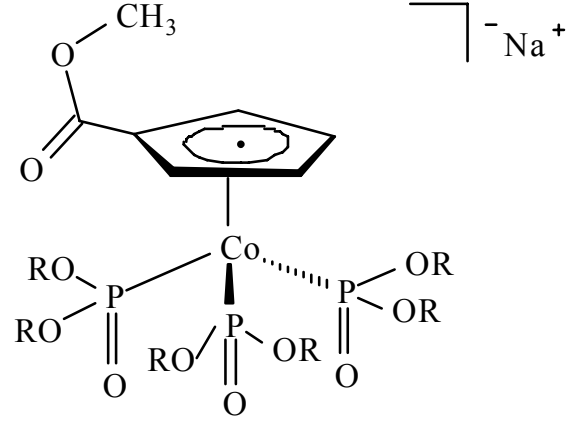

Figure 2.1. Methyl Ester Derivative of the Kläui Ligand $\left(\mathrm{R}=\mathrm{CH}_{2} \mathrm{CH}_{3}\right)$ in coloration of the starting metallocene solution from red to a dark yellow-brown. Removing excess diethylphosphite in vacuo resulted in a dark yellow-brown oil, which was difficult to purify further. The FTIR spectrum of this crude product showed a strong $v(\mathrm{C}=\mathrm{O})$ band at $1707 \mathrm{~cm}^{-1}$ corresponding to an ester carbonyl functionality. The expected molar mass of 2 is $1245.6 \mathrm{amu}$. The electrospray-ionization mass spectrum of the crude product showed an $\mathrm{m} / \mathrm{z}$ ratio of 1242.63-1246.60 amu, which is near the expected value of the molecular mass of 2.

Crystals were grown from the crude product containing 2 by slow diffusion of pentane into a concentrated ether solution. Large translucent crystals slowly formed over a period of approximately a week and were used for single-crystal x-ray diffraction study; the structure of the compound that crystallized out of solution is shown in Figure 2.3. Somewhat surprisingly, the structure revealed that the ester group had been reduced to a methyl group on the $\mathrm{Cp}$ ring, apparently as a result of reduction of the ester by the diethylphosphite. Alternatively, it is possible that the compound is not thermally stable and decarbonylated at the relatively high temperatures of refluxing diethylphosphite. The FTIR spectrum of the isolated crystals lacked the $v(\mathrm{C}=\mathrm{O})$ band for the ester group that was observed in the crude product of 2. 


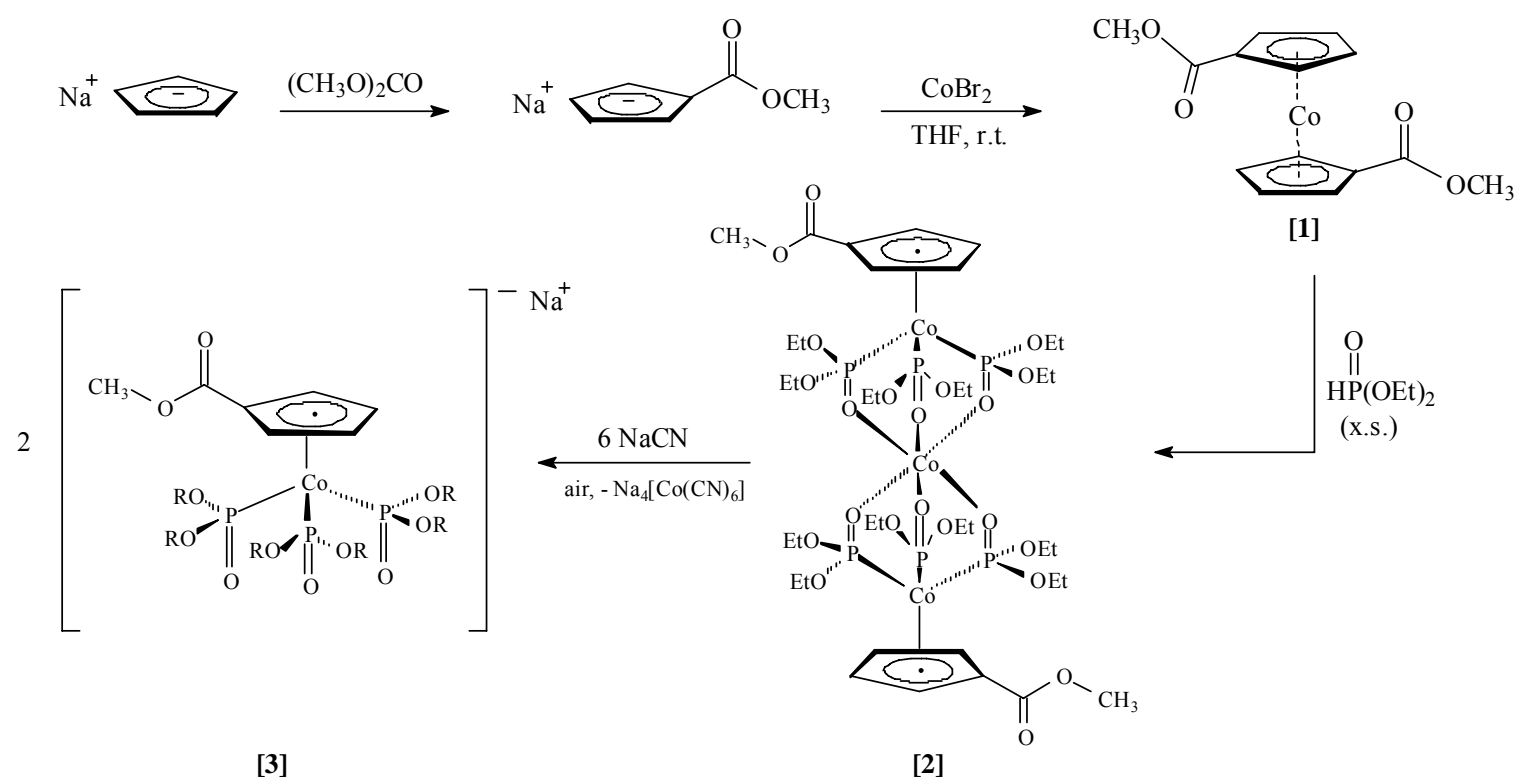

Figure 2.2. Synthetic Scheme for Functionalizing Kläui Ligands

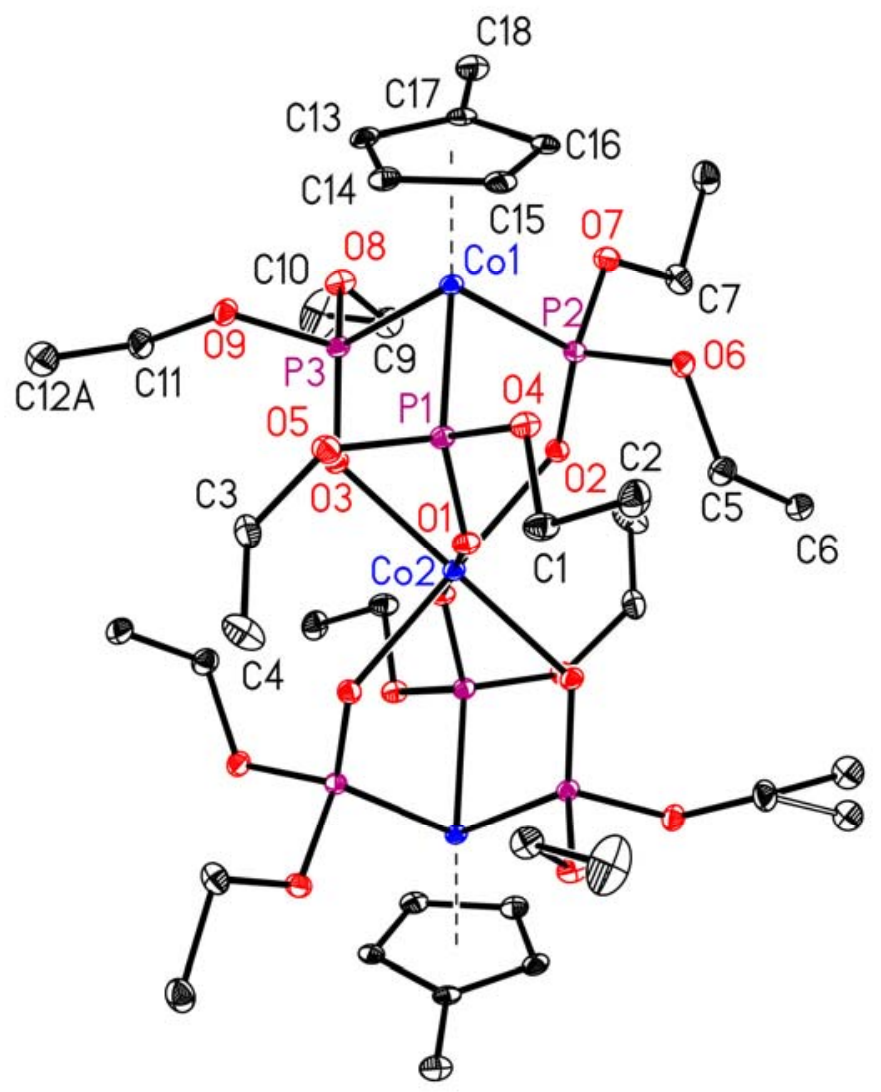

Figure 2.3. ORTEP Plot of the Crystals Obtained from Crystallization of Product Containing Compound 2; Note the Reduction of the Ester Groups to Methyl Groups on the Cp Ring 
Compound 3 was prepared using a procedure first reported by Román and co-workers. (Román et al. 1986). A solution of the crude product containing 2 (and probably the methyl Cp derivative as well) was mixed with $\mathrm{NaCN}$ in methanol and stirred in air for 2 hours until the product was soluble in methanol. The original orange solution turned yellow over the course of the reaction. Evaporation of the methanol afforded a dark yellow oil. Attempts to crystallize the product from this reaction failed. The FTIR spectrum of the oily product displayed a strong $v(\mathrm{C}=\mathrm{O})$ band at $1700 \mathrm{~cm}^{-1}$, which is supportive of the presence of the expected ester functionality on the Kläui ligand (sodium salt); however, it is likely that this product is impure and contains the corresponding methyl derivative as well.

In summary, the synthetic work conducted in FY 2006 demonstrated the feasibility of preparing Kläui ligands derivatized at the $\mathrm{Cp}$ ring. The reduction of the carbonyl functional groups by the diethylphosphite reagent complicates purification of the desired products. To overcome the consequences of this side reaction, an alternative approach should be taken to synthesize the polymers functionalized with Kläui ligands. Such an approach is outlined in Figure 2.4. To prepare the intermediate Kläui complex with three cobalt atoms, it would be easier to prepare and purify 1,1'-dimethylcobaltocene. The methyl groups can then be oxidized to carboxylic acid groups using a mild oxidation procedure and subsequently converted to the respective acyl chloride compound using oxalyl chloride. Reaction with an amine functionalized polymer would tether the Kläui ligands to the polymer. A final oxidative cyanidation step would complete the synthesis (two Kläui ligands may be attached to the polymer for every triple-decker compound reacted with the polymer). This approach will be pursued in FY 2007.

The bicyclic malonamide (BMA) ligands were synthesized for this project according to procedures developed at the University of Oregon. As with the Kläui ligands, incorporating a terminal functional group such as a carboxylic acid $(\mathrm{COOH})$ or thiol $(\mathrm{SH})$ is necessary for these materials to be directly coupled to solid supports. Once these groups are present, the ligands can be immobilized onto a compatible solid support such as polymer resins or mesoporous silica using well-established coupling protocols. To date, the majority of our efforts have been placed on synthesizing and characterizing the carboxylate-containing BMA with symmetric substitution (Figure 2.5). The symmetrically substituted materials are the easier synthetic targets so were synthesized first for use in this project. Work has been initiated on synthesizing the asymmetric materials (Figure 2.5), and this work will continue in FY07.

It is worth mentioning that the BMA ligands typically possess an ester protecting group at the carboxylate moiety that needs to either be cleaved before immobilization onto the solid support, or an ester-reactive functionality (e.g., oxime) must be incorporated into the support before coupling. For coupling the symmetrically substituted BMAs during this project, we have chosen a PL-Oxime resin from PolymerLabs (Figure 2.6) that is reactive toward carboxylic acids as well as ethyl, t-butyl, and methyl esters. These resins will allow us to produce loading densities comparable to those typically achieved on commercially available ion exchange resins. One advantage of using a resin that is reactive toward esters is that it eliminates the need to deprotect the molecule before immobilization. Starting in FY07, we will develop the chemistry necessary to couple the symmetrically substituted materials to the oxime resins. In addition, work will be continued to develop the synthesis of the asymmetrically substituted BMAs. These materials have the advantage that there is only one functional group available for attachment to a surface, which should lead to a situation in which the BMA ligands are bound to the resin in an orientation favorable for further binding to the radionuclide target. In addition, the asymmetrically substituted material will not have the same propensity to crosslink the individual resin beads leading to larger aggregate materials. This point also makes the asymmetric materials ideal for coupling to alternative supports such as mesoporous silica and magnetic nanoparticles. 

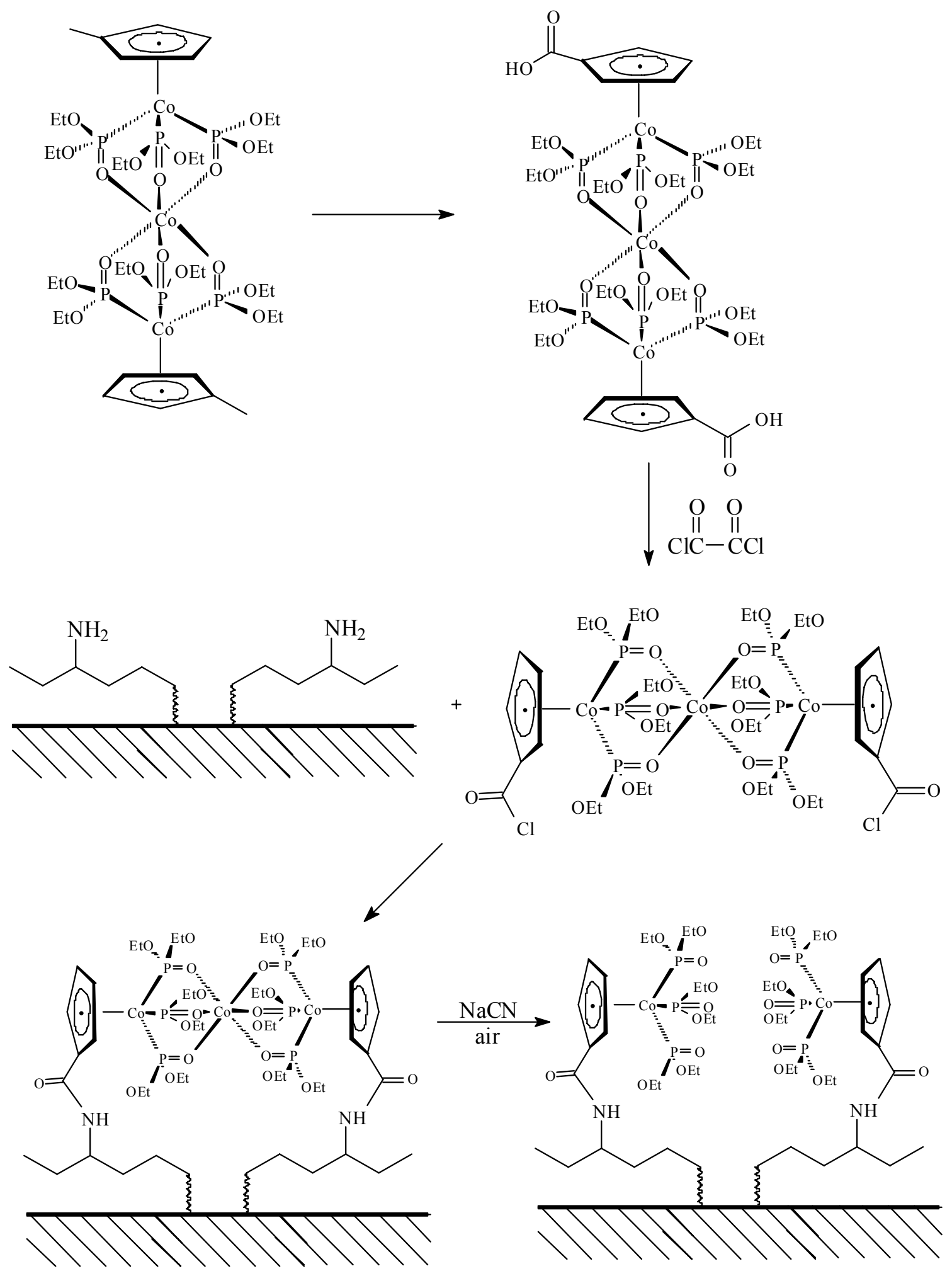

Figure 2.4. Schematic Depiction of a Functionalized Kläui Ligand Covalently Bound to an Organic Resin 


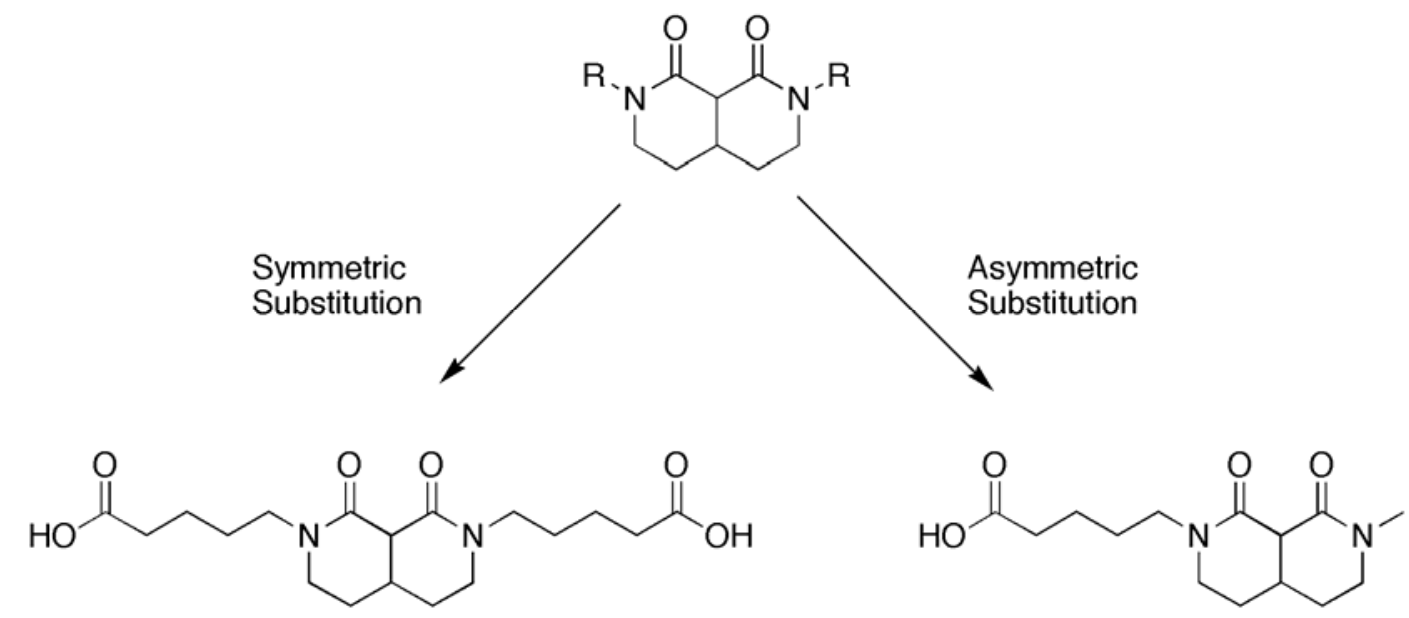

Figure 2.5. Structure of BMA Ligands Showing Symmetric and Asymmetric Substitution of the $-\mathrm{COOH}$ Containing Functionality

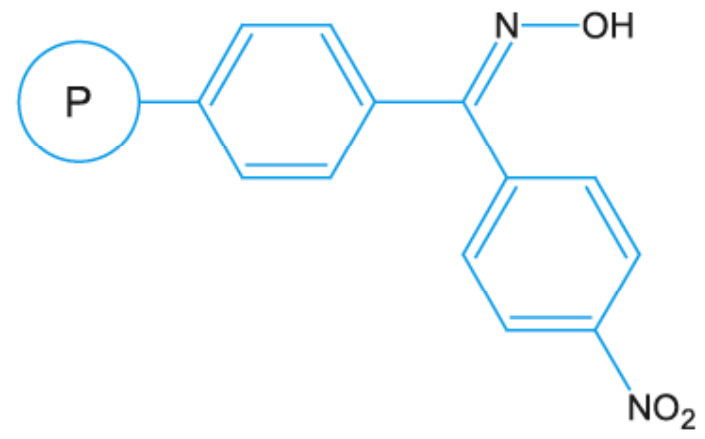

Figure 2.6. Illustration of the Functionality Present on the Surface of the PL-Oxime Resin 


\subsection{Separations Materials Evaluation}

While the synthetic efforts described in Section 2.0 were in progress, a number of new separations media were already available to evaluate potential application in automated actinide separations. These included two extraction chromatography resins containing Kläui ligands $1 \mathrm{~b}$ and 1c (Figure 1.2) and selfassembled monolayer on mesoporous supports (SAMMS) materials containing diphosphonate groups. The Kläui ligand resins contained either $0.75 \mathrm{wt} \% 1 \mathrm{~b}$ or $1.0 \mathrm{wt} \% 1 \mathrm{c}$ sorbed onto Amberlite ${ }^{\circledR}$ XAD-7HP (Lumetta et al. 2004). In this discussion, these are referred to as the "Et-Kläui" resin or the "Pr-Kläui" resin, respectively. Batch equilibrium distribution measurements for various actinide ions were made for the ${ }^{n} \mathrm{Pr}-\mathrm{Kläui}$ resin and the SAMMS materials. In addition, experiments were performed to investigate methods to recover Pu from the Kläui resins. The following subsections describe the results of these investigations.

\subsection{Batch Equilibrium Distribution Experiments with the ${ }^{\mathrm{n}}$ Pr-Kläui Resin}

Four major factors largely determine actinide sorption efficiency by the ${ }^{\mathrm{n}} \operatorname{Pr}-\mathrm{Kläui}$ resin. These are 1) the coordination mechanism, 2) the complex stoichiometry, 3) aqueous acid concentration, and 4) competing ions present in the aqueous phase. Each of these factors is discussed individually below.

Coordination mechanism. In an alkyl Kläui ligand, three phosphate atoms (with oxygen electron donors) arranged in a tripod-like configuration are available for metal ion coordination (Figure 1.2). The efficiency and mechanism of this coordination depend upon electronic and spatial complementarity between the binding moiety of the ligand and the metal ion, which in turn depends on the oxidation state of the metal ion as well as its aqueous speciation. Molecular modeling calculations have suggested that tri- and tetravalent actinides, which are characterized by high positive charge density and mobile inner coordination sphere environment, should strongly coordinate to all three phosphate oxygens of the Kläui ligand (Lumetta et al. 2006). On the other hand, the pentavalent and hexavalent actinides in the form of the linear trans-dioxo ions like $\mathrm{UO}_{2}{ }^{2+}$ prefer the pentagonal or hexagonal planar coordination environment, which is not complementary with the tripodal Kläui ligand. Accordingly, the efficiency of the actinide sorption by the ${ }^{n} \mathrm{Pr}-\mathrm{Kläui}$ resin is expected to be different for actinides in different oxidation states.

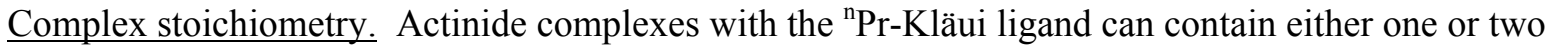
${ }^{n} \operatorname{Pr}-\mathrm{Kläui}$ ligands per actinide center. The stoichiometry of the complex is largely determined by the ligand loading (i.e., the amount of metal ion loaded onto the resin compared with the number of ligands available for binding). At low ligand loading, both 1:1 and 1:2 stoichiometries are feasible, while at high ligand loading, the lack of available ligand would be expected to promote the formation of 1:1 complexes.

Aqueous acid concentration. The ${ }^{\mathrm{n}} \mathrm{Pr}-\mathrm{Kläui}$ ligand possesses a negative charge of unity and is deposited on the resin as the sodium salt. Upon contact with an acidic solution, it can be converted into the protonated form according to Equation 3.1.

$$
\mathrm{H}^{+}{ }_{\text {aq }}+(\text { Pr-Kläui }) \mathrm{Na}_{\text {resin }} \Leftrightarrow \mathrm{Na}_{\text {aq }}^{+}+\left(\text {Pr-Kläui) } \mathrm{H}_{\text {resin }}\right.
$$

This also implies that when a metal ion is coordinated by the phosphate functional group of the ligand, the simultaneous process of proton exchange by the metal ion takes place. Thus, an increase of aqueous $\mathrm{HNO}_{3}$ concentration makes ion exchange process more difficult. The coordination process, as 
described by Equations 3.2 and 3.3, also involves counteranions, e.g., nitrate, to maintain electroneutrality of the multivalent actinides; and the increase of aqueous $\mathrm{HNO}_{3}$ concentration brings about change of aqueous speciation of the metal ion; combining these processes is expected to affect sorption efficiency in a non-linear manner.

$$
\begin{gathered}
\mathrm{M}^{\mathrm{n}+}{ }_{\text {aq }}+\mathrm{n}\left(\mathrm{NO}_{3}{ }^{-}\right)_{\mathrm{aq}}+(\operatorname{Pr}-\mathrm{Kläui}) \mathrm{H}_{\mathrm{resin}} \Leftrightarrow \mathrm{H}^{+}{ }_{\mathrm{aq}}+\left(\mathrm{M}^{\mathrm{n}+}\right)(\mathrm{Pr}-\mathrm{Kläui})\left(\mathrm{NO}_{3}{ }^{-}\right)_{(\mathrm{n}-1) \mathrm{resin}} \\
\mathrm{M}^{\mathrm{n}+}{ }_{\mathrm{aq}}+\mathrm{n}\left(\mathrm{NO}_{3}{ }^{-}\right)_{\mathrm{aq}}+2(\mathrm{Pr}-\mathrm{Kläui}) \mathrm{H}_{\mathrm{resin}} \Leftrightarrow 2 \mathrm{H}_{\mathrm{aq}}^{+}+\left(\mathrm{M}^{\mathrm{n}+}\right)(\mathrm{Pr}-\mathrm{Kläui})_{2}\left(\mathrm{NO}_{3}{ }^{-}\right)_{(\mathrm{n}-2) \text { resin }}
\end{gathered}
$$

Composition of the aqueous phase. Composition of the aqueous phase affects actinide sorption via the presence of competing ions capable of coordinating to a Kläui ligand or affecting the aqueous actinide speciation. Thus, understanding of metal sorption behavior pattern exhibited by the ${ }^{\mathrm{n}} \mathrm{Pr}-\mathrm{Kläui}$ resin requires a multi-parametric study, which was beyond the scope of this investigation. But future experiments should be performed to assess the influence of competing ions on the sorption of the actinide ions on the Kläui resins.

A number of batch equilibrium distribution experiments were performed using the ${ }^{n} \mathrm{Pr}-\mathrm{Kläui}$ resin. These included experiments to investigate 1) the acid dependence of sorption, 2) the effect of initial $\mathrm{Pu}$ concentration, and 3) the effect of ligand loading. Batch distribution coefficients were determined via equilibration of a weighed amount ( 0.03 to $0.05 \mathrm{~g}$ ) of the ${ }^{\mathrm{n}} \mathrm{Pr}-\mathrm{Kläui}$ resin with a measured volume (2 to $3 \mathrm{~mL}$ ) of the $\mathrm{HNO}_{3}$ solution containing a known concentration of the metal ion. Measurements were performed in duplicate for each experimental condition. Equilibration was done using 2-dram (7 mL) glass scintillation vials shaken in an oscillating mode for 17 to $20 \mathrm{~h}$. Preliminary kinetics measurements indicated this length of time was sufficient to achieve equilibrium. The temperature was monitored and demonstrated to be $22 \pm 1^{\circ} \mathrm{C}$. After equilibration, solution aliquots were withdrawn for analyses.

To determine the metal ion distribution ratio $\left(K_{\mathrm{d}}\right)$, the activity of actinide was measured by an alphaor a gamma-counting technique. Typically, alpha activity was determined by liquid scintillation counting (LSC) using $5 \mathrm{~mL}$ of Ultima Gold XR liquid scintillation cocktail (Packard BioScience, Meriden, CN). The relative alpha activity of the samples was determined by LSC using a Packard Tri-Carb 2260XL. The activity of ${ }^{155} \mathrm{Eu}$ was measured by gamma-counting using a 3-inch $\mathrm{NaI}(\mathrm{Tl})$ crystal through-hole type detector (Packard Cobra Quantum Model 5003). Gamma and LSC counting results were corrected for background, determined by counting non-radioactive controls under identical conditions. $K_{\mathrm{d}}$ values $(\mathrm{mL} / \mathrm{g})$ were calculated using Equation 3.4,

$$
K_{\mathrm{d}}=\frac{A_{0}-A_{\mathrm{f}}}{A_{\mathrm{f}}} \times \frac{V}{W}
$$

where $A_{0}=$ the initial activity of the test solution in counts per minute (cpm)

$A_{\mathrm{f}}=$ final activity of the test solution in cpm

$V=$ test solution volume in $\mathrm{mL}$

$W=$ mass of the dry sorbent.

Two kinds of batch contact experiments were performed. To determine the dependence of metal ion sorption on the acid concentration, experiments entailed variation of the nitric acid concentration in the 0.1 to $8 \mathrm{M}$ range at a fixed initial metal ion concentration in the aqueous solution. In the experiments that 
were performed to evaluate the effect of Kläui ligand loading on the sorption, the nitric acid concentration was fixed at $0.3 \mathrm{M}$, and the metal ion concentration systematically varied. For $\mathrm{Pu}(\mathrm{IV})$, this experiment was performed at $1 \mathrm{M} \mathrm{HNO}_{3}$ as well. The Kläui ligand loading was calculated using Equation 3.5.

$$
\text { Ligand Loading }=\frac{\text { moles }(\text { metal sorbed })}{\text { moles }(\text { ligand total })}
$$

\subsection{Acid Dependence of Actinide(III, IV, V, and VI) and Eu(III) Sorption by the ${ }^{\mathrm{n}}$ Pr-Kläui Resin}

Figure 3.1 illustrates the effect of the $\mathrm{HNO}_{3}$ concentration on actinide (III, IV, V, and VI) and $\mathrm{Eu}(\mathrm{III})$ sorption on the ${ }^{\mathrm{n}} \mathrm{Pr}-\mathrm{Kläui}$ resin. Control experiments were performed for each metal ion under identical conditions using Amberlite XAD-7HP sorbent containing no ligand. Negligible sorption of the investigated metal ions on the blank resin confirmed that in the 0.1 to $10 \mathrm{M} \mathrm{HNO}_{3}$ concentration range, metal ion sorption is due to metal complexation by the ${ }^{\mathrm{n}} \mathrm{Pr}-\mathrm{Kläui}$ ligand. It was found that sorption efficiency and its dependence on the aqueous $\mathrm{HNO}_{3}$ concentration was highly dependent upon the oxidation state of the actinide ion.

The ${ }^{\mathrm{n}} \mathrm{Pr}-\mathrm{Kläui}$ resin exhibited the strongest affinity for the tetravalent actinides. It was found that at $1 \mathrm{M} \mathrm{HNO}_{3}$, the sorption of $\mathrm{Pu}(\mathrm{IV})$ on the ${ }^{\mathrm{n}} \mathrm{Pr}-\mathrm{Kläui}$ resin was about 50 times higher than that for U(VI). The separation factor for $\mathrm{Pu}(\mathrm{IV})$ over $\mathrm{Am}$ (III) under these conditions was 23000 . Exceptionally high affinity was observed for $\mathrm{Np}(\mathrm{IV})$. It is worth noting that in this experiment, the initial concentration of $\mathrm{Np}$ (IV) $\left(7.8 \times 10^{-5} \mathrm{M}\right)$ was greater than that of $\mathrm{Pu}(\mathrm{IV})\left(2.1 \times 10^{-5} \mathrm{M}\right)$, resulting in 5 times greater loading of the ${ }^{\mathrm{n}} \mathrm{Pr}-\mathrm{Kläui}$ ligand with $\mathrm{Np}(\mathrm{IV})$, which was about $55 \%$ throughout the entire $\mathrm{HNO}_{3}$ concentration range. Based on the trend for decreasing $K_{\mathrm{d}}$ with increasing loading (vide infra), it can be expected that under identical loading conditions, the differences between $\mathrm{Np}$ (IV) and $\mathrm{Pu}(\mathrm{IV}$ ) sorption would be even greater. The initial nearly linear decrease in $K_{\mathrm{d}}$ values for $\mathrm{Pu}(\mathrm{IV})$ and $\mathrm{Np}(\mathrm{IV})$ with increasing $\left[\mathrm{HNO}_{3}\right]$ could be explained by competitive protonation of the ligand as well as the corresponding change of the aqueous speciation of $\mathrm{M}(\mathrm{IV})$. When $\mathrm{HNO}_{3}$ concentration increases from 0.2 to $1 \mathrm{M}$, equilibrium reactions 3.6 and 3.7 must be considered. At low acidity, reaction 3.6 dominates. Hydrolysis of M(IV) occurs as well,

$$
\begin{gathered}
\mathrm{Pu}^{4+}+\mathrm{NO}_{3}{ }^{-} \Leftrightarrow \mathrm{Pu}\left(\mathrm{NO}_{3}\right)^{3+} \\
\mathrm{Pu}\left(\mathrm{NO}_{3}\right)^{3+}+\mathrm{NO}_{3}{ }^{-} \Leftrightarrow \mathrm{Pu}\left(\mathrm{NO}_{3}\right)_{2}{ }^{2+}
\end{gathered}
$$

but its effect on sorption is not understood sufficiently. When the $\mathrm{HNO}_{3}$ concentration increases from 0.2 to $1 \mathrm{M}$, the relative content of species $\mathrm{Pu}\left(\mathrm{NO}_{3}\right)^{3+}$ and $\mathrm{Pu}\left(\mathrm{NO}_{3}\right)_{2}{ }^{2+}$ changes, and species $\mathrm{Pu}\left(\mathrm{NO}_{3}\right)_{2}{ }^{2+}$ becomes dominant at $1 \mathrm{M} \mathrm{HNO}_{3}$. This aqueous speciation change is reflected by a decline in the sorption of $\mathrm{Pu}(\mathrm{IV})$. The sorption plateau in the 1 to $5 \mathrm{M} \mathrm{HNO}_{3}$ concentration range corresponds to the dominant $\mathrm{Pu}\left(\mathrm{NO}_{3}\right)_{2}{ }^{2+}$ species. At higher $\mathrm{HNO}_{3}$ concentrations, tetra- and hexa-nitrato complex species of general formula $\left[\mathrm{M}\left(\mathrm{NO}_{3}\right)_{6}\right]^{2-}$ are preferentially formed according to Equations 3.8 and 3.9.

$$
\mathrm{Pu}\left(\mathrm{NO}_{3}\right)_{2}{ }^{2+}+2 \mathrm{NO}_{3}^{-} \Leftrightarrow \mathrm{Pu}\left(\mathrm{NO}_{3}\right)_{4}
$$




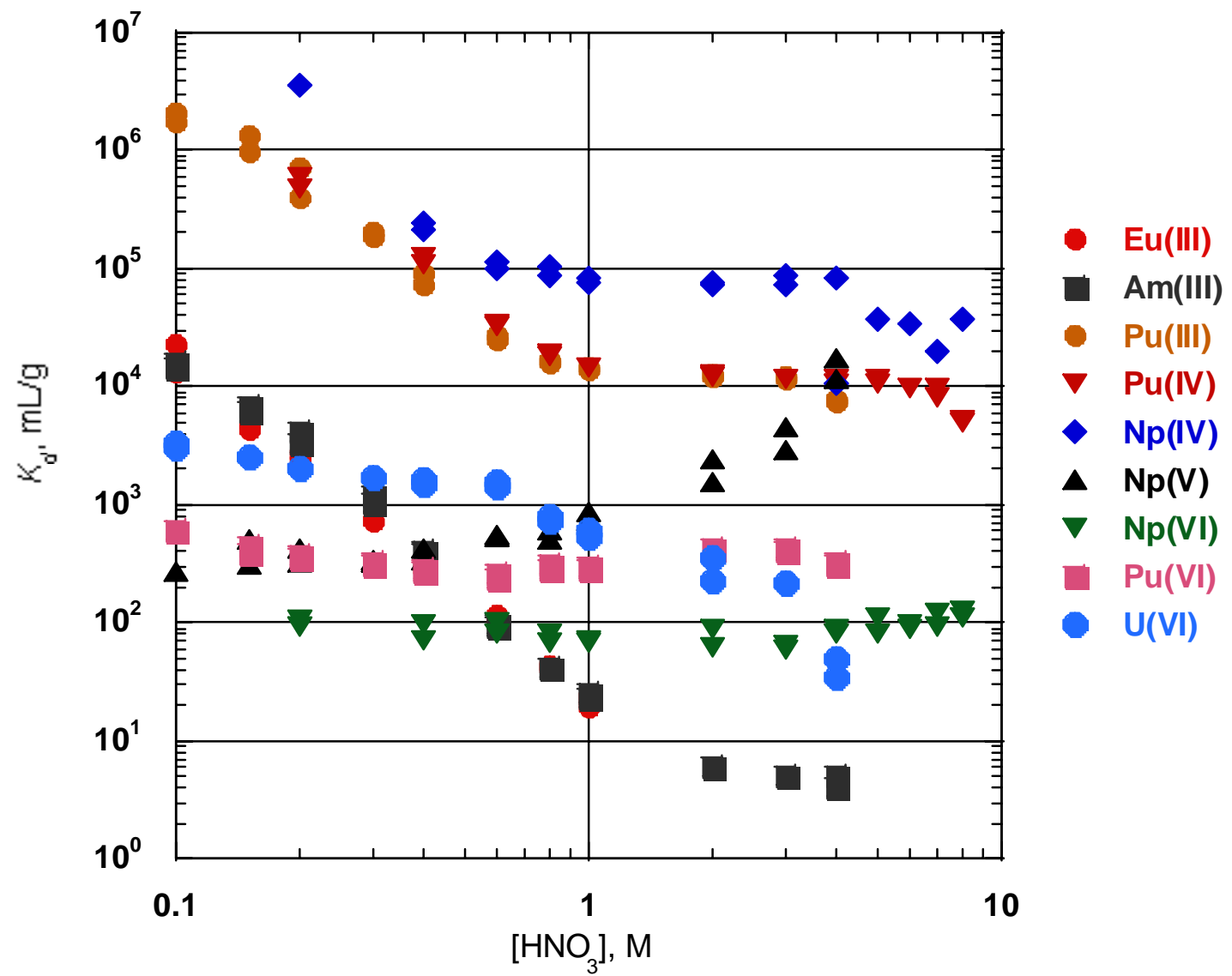

Figure 3.1. Acid Dependence of Actinide (III, IV, V, and VI) and Eu(III) Sorption by the ${ }^{\mathrm{n}} \mathrm{Pr}-\mathrm{Kläui}$ Resin ( $30 \mathrm{mg}) . K_{\mathrm{d}}$ values were measured using aqueous solutions of fixed metal ion concentration as a function of $\mathrm{HNO}_{3}$ concentration. Initial metal ion concentration was $2.0 \times 10^{-6} \mathrm{M}$ for $\mathrm{Eu}(\mathrm{III}), 1.8 \times 10^{-6} \mathrm{M}$ for $\mathrm{Am}(\mathrm{III}), 1.6 \times 10^{-5} \mathrm{M}$ for $\mathrm{Pu}(\mathrm{III}), 2.1 \times 10^{-5} \mathrm{M}$ for $\mathrm{Pu}(\mathrm{IV}), 7.8 \times 10^{-5} \mathrm{M}$ for $\mathrm{Np}(\mathrm{IV}), 2.8 \times 10^{-5} \mathrm{M}$ for $\mathrm{Np}(\mathrm{V}), 4.4 \times 10^{-5} \mathrm{M}$ for $\mathrm{Np}(\mathrm{VI}), 2.0 \times 10^{-5} \mathrm{M}$ for $\mathrm{Pu}(\mathrm{VI})$, and $1.34 \times 10^{-5} \mathrm{M}$ for $\mathrm{U}(\mathrm{VI})$. Maximum loading was $0.9 \%$ for $\mathrm{Eu}(\mathrm{III}), 0.9 \%$ for $\mathrm{Am}(\mathrm{III}), 7.8 \%$ for $\mathrm{Pu}(\mathrm{III}), 10 \%$ for $\mathrm{Pu}(\mathrm{IV}), 55 \%$ for $\mathrm{Np}(\mathrm{IV}), 12.8 \%$ for $\mathrm{Np}(\mathrm{V}), 18 \%$ for $\mathrm{Np}(\mathrm{VI}), 8 \%$ for $\mathrm{Pu}(\mathrm{VI})$, and $6 \%$ for $\mathrm{U}(\mathrm{VI})$.

$$
\mathrm{Pu}\left(\mathrm{NO}_{3}\right)_{4}+2 \mathrm{NO}_{3}^{-} \Leftrightarrow \mathrm{Pu}\left(\mathrm{NO}_{3}\right)_{6}^{2-}
$$

The yield of the $\mathrm{Pu}\left(\mathrm{NO}_{3}\right)_{6}{ }^{2-}$ species depends upon the nitric acid concentration in the solution and increases from 5 to $90 \%$ in the 5 to $10 \mathrm{M} \mathrm{HNO}_{3}$ concentration range (Ryan 1960). Hexanitrato complex species are negatively charged and, thus, are not expected to interact directly with the Kläui ligand, lowering effective $\mathrm{M}(\mathrm{IV})$ sorption at $\mathrm{HNO}_{3}$ concentrations greater than $5 \mathrm{M}$. The comparable shape of the $\mathrm{Np}(\mathrm{IV})$ and $\mathrm{Pu}(\mathrm{IV})$ sorption isotherms suggests similar interaction mechanisms for these metal ions with the ${ }^{\mathrm{n}} \mathrm{Pr}-\mathrm{Kläui}$ ligand.

Americium(III) and Eu(III) exhibited nearly identical sorption, indicating the same mechanism of their interaction with ${ }^{\mathrm{n}} \mathrm{Pr}-\mathrm{Kläui}$ ligand and similar binding affinity. Strong sorption of Am(III) and Eu(III) 
to the ${ }^{\mathrm{n}} \mathrm{Pr}-\mathrm{Kläui}$ resin was observed at low nitric acid concentrations. Their sorption linearly decreased with increasing $\mathrm{HNO}_{3}$ concentration and became negligible at $2 \mathrm{M} \mathrm{HNO}_{3}$. This can be explained by the unfavorable $\mathrm{M}(\mathrm{III})$ competition with the protonation reaction (Equation 3.1). On the other hand, $\mathrm{Pu}(\mathrm{III}$ ) exhibited drastically different sorption behavior. The sorption of $\mathrm{Pu}(\mathrm{III})$ was very similar to that of $\mathrm{Pu}(\mathrm{IV})$. This observation and UV-vis measurements of Pu-loaded ${ }^{\mathrm{n}} \mathrm{Pr}-\mathrm{Kläui}$ resin suggest that $\mathrm{Pu}(\mathrm{III})$ sorption is accompanied by the redox reaction $\mathrm{Pu}(\mathrm{III}) \rightarrow \mathrm{Pu}(\mathrm{IV})$, so that $\mathrm{Pu}(\mathrm{IV})$ is deposited on the resin. Presumably, the much stronger preference of the ${ }^{n} \mathrm{Pr}-\mathrm{Kläui}$ ligand for the tetravalent actinides lowers the energy barrier of this redox process and thermodynamically drives $\mathrm{Pu}(\mathrm{IV})$ sorption. Similar behavior was observed for $\mathrm{Np}(\mathrm{V})$, which was reduced to $\mathrm{Np}(\mathrm{IV})$ upon coordination to the Pr-Kläui ligand. The UV-vis spectrum of the $\mathrm{Np}(\mathrm{V})$-loaded ${ }^{\mathrm{n}} \mathrm{Pr}-\mathrm{Kläui}$ resin contained no spectral features characteristic of $\mathrm{Np}(\mathrm{V})$, but rather was very similar to the spectrum of the $\mathrm{Np}$ (IV)-loaded ${ }^{\mathrm{n}} \mathrm{Pr}$-Kläui resin (Figure 3.2). The increase of the $K_{d}$ values for $\mathrm{Np}(\mathrm{V})$ with acid concentration (Figure 3.1) was attributed to this $\mathrm{Np}(\mathrm{V}) \rightarrow \mathrm{Np}(\mathrm{IV}$ ) redox process, which was promoted at elevated $\mathrm{HNO}_{3}$ concentrations. At low $\mathrm{HNO}_{3}$ concentrations, the sorption of $\mathrm{Np}(\mathrm{V})$ was about 1.5 orders-of-magnitude lower. Further investigation is needed to elucidate the mechanism of the redox processes involving actinides in the presence of the Kläui ligands.
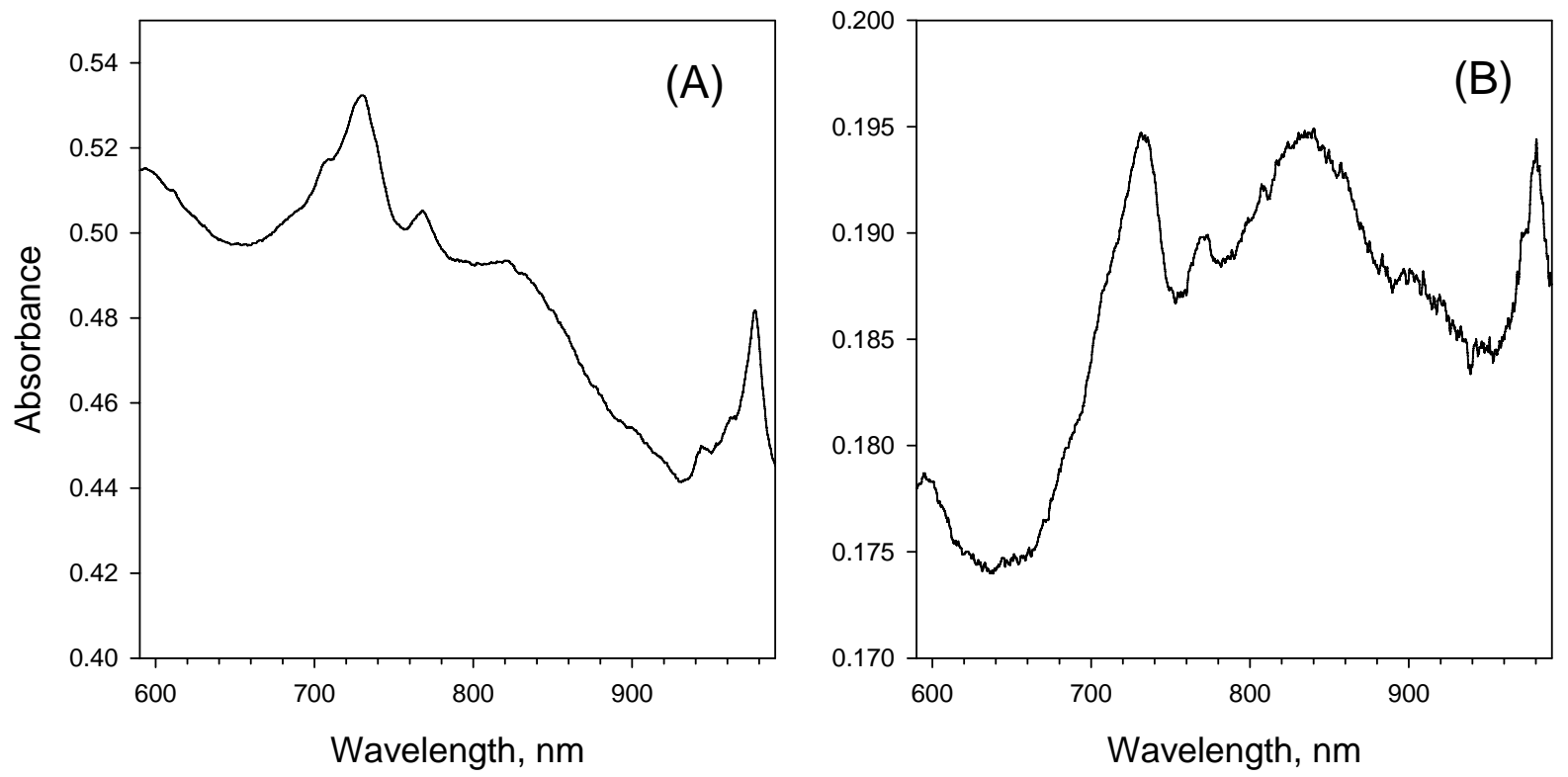

Figure 3.2. UV-vis Spectra of the ${ }^{\mathrm{n}} \mathrm{Pr}-\mathrm{Kläui}$ Resin Equilibrated with $\mathrm{Np}(\mathrm{IV})(\mathrm{A})$ and $\mathrm{Np}(\mathrm{V})(\mathrm{B})$ Solutions in $0.1 \mathrm{M} \mathrm{HNO}_{3}$

Neptunium(VI), Pu(VI), and U(VI) exhibited relatively low sorption in the entire nitric acid concentration range (Figure 3.1). This low sorption of the hexavalent actinides can be explained by the geometrical mismatch of the tripodal Kläui ligand and the equatorial pentagonal or hexagonal planar coordination environment preferred by the linear trans-dioxo ions like $\mathrm{UO}_{2}{ }^{2+}$. Uranium(VI) sorption gradually declined as $\mathrm{HNO}_{3}$ concentration increased, which is likely because of competitive protonation of the ligand, but it is surprising that the retention of $\mathrm{U}(\mathrm{VI})$ on the resin becomes higher than that for $\mathrm{Am}(\mathrm{III})$ and $\mathrm{Eu}(\mathrm{III})$ at high $\left[\mathrm{HNO}_{3}\right]$ despite the assumed geometrical mismatch between the $\mathrm{UO}_{2}{ }^{2+}$ ion and the tripodal Kläui ligand. The sorption of $\mathrm{Np}(\mathrm{VI})$ and $\mathrm{Pu}(\mathrm{VI})$ was observed to increase slightly with the acid concentration. Speculatively, it can be explained by the reduction of these actinides to tetravalent 
$\mathrm{Np}(\mathrm{IV})$ and $\mathrm{Pu}(\mathrm{IV})$, respectively, which have the greatest affinity to the Kläui ligand. Because of the larger redox barrier of the stable uranyl ion, the same redox process did not occur for U(VI).

\subsubsection{Effect of Initial Aqueous Pu(IV) Concentration on Pu(IV) Sorption by ${ }^{\mathrm{n}} \mathrm{Pr}-\mathrm{Kläui}$ Resin}

The nitric acid dependence of the $\mathrm{Pu}(\mathrm{IV})$ sorption was studied at three different initial concentrations of $\mathrm{Pu}\left(\mathrm{IV}\right.$ ) (Figure 3.3). The initial $\mathrm{Pu}(\mathrm{IV})$ concentrations were $2.13 \times 10^{-5}, 8.50 \times 10^{-5}$, and $1.11 \times 10^{-4} \mathrm{M}$, which corresponded to $10,2.6$, and 1.3 times molar excess of Kläui ligand over $\mathrm{Pu}(\mathrm{IV})$ in the batch contact systems, respectively. The resultant ligand loading increased from 10 to about $70 \%$ as ligand excess in the system decreased. Increasing ligand loading dramatically affected $\mathrm{Pu}(\mathrm{IV})$ sorption at low $\mathrm{HNO}_{3}$ concentration, so that $K_{\mathrm{d}}$ values dropped three orders-of-magnitude at $0.2 \mathrm{M} \mathrm{HNO}_{3}$ in going from the low- to high-loading condition. The shapes of the sorption isotherms using $2.13 \times 10^{-5}$ and $8.50 \times 10^{-5}$ $\mathrm{M} \mathrm{Pu}(\mathrm{IV})$ solutions were similar, suggesting that the corresponding increase of ligand loading from 10 to $38 \%$ did not change the sorption mechanism. It was also accompanied by moderate sorption reduction. As it is seen in Figure 3.3, $K_{\mathrm{d}}$ values decrease in the nitric acid concentration range of 0.2 to $1 \mathrm{M}$, presumably because of the increasingly unfavorable competition with $\mathrm{H}^{+}$; then leveling off at the 1 to $5 \mathrm{M}$ $\mathrm{HNO}_{3}$; and finally dropping again at an $\mathrm{HNO}_{3}$ concentration greater than $5 \mathrm{M}$ because of the formation of hexanitrato $\mathrm{Pu}(\mathrm{IV})$ complex species (Equations 3.8 and 3.9). On the other hand, the shape of the sorption isotherm corresponding to the $1.11 \times 10^{-4} \mathrm{M} \mathrm{Pu}(\mathrm{IV})$ solution is very different. The $K_{\mathrm{d}}$ values increase about one order of magnitude as the $\mathrm{HNO}_{3}$ concentration increases and converge with $K_{\mathrm{d}}$ values obtained using $2.13 \times 10^{-5}$ and $8.50 \times 10^{-5} \mathrm{M} \mathrm{Pu}(\mathrm{IV})$ solutions at $5 \mathrm{M} \mathrm{HNO}_{3}$. The mechanistic explanation for this behavior is not currently understood.

\subsubsection{Selectivity and Effect of ${ }^{\text {n}}$ Pr-Kläui Resin Loading on Actinide (III, IV, V, and VI) and Eu(III) Sorption}

The selectivity of $\mathrm{Pu}(\mathrm{IV})$ sorption by the ${ }^{\mathrm{n}} \mathrm{Pr}-\mathrm{Kläui}$ resin is determined primarily by the aqueous concentration of the $\mathrm{HNO}_{3}$ and the ligand loading. To eliminate the effect of varying $\mathrm{HNO}_{3}$, the $K_{\mathrm{d}}$ values for various actinides and $\mathrm{Eu}(\mathrm{III})$ were determined as a function of initial metal ion concentration at a constant $\mathrm{HNO}_{3}$ concentration of $0.3 \mathrm{M}$. The resulting $K_{\mathrm{d}}$ values were plotted against the initial metal ion concentration and resulting ligand loading (Figure 3.4). The data generated in this way present a true indication of the selectivity of the ${ }^{\mathrm{n}} \mathrm{Pr}-\mathrm{Kläui}$ ligand for the actinide ions and $\mathrm{Eu}(\mathrm{III})$ in $0.3 \mathrm{M} \mathrm{HNO}_{3}$. The results of these measurements confirmed the previously observed high affinity of the Kläui ligands for tetravalent actinide ions. At a ligand loading of 7\%, the separation factors for $\mathrm{Pu}(\mathrm{IV})$ over $\mathrm{Eu}(\mathrm{III})$, $\mathrm{Np}(\mathrm{V}), \mathrm{Pu}(\mathrm{VI})$, and $\mathrm{U}(\mathrm{VI})$ were found to be $750,800,1000$, and 270 , respectively.

The effect of ligand loading was the most pronounced for $\mathrm{Pu}(\mathrm{IV})$; the corresponding $K_{\mathrm{d}}$ values decreased 1.5 orders-of-magnitude when ligand loading increased from 5 to $50 \%$. The further increase of ligand loading up to $65 \%$ caused an additional decrease of the $K_{\mathrm{d}}$ values for $\mathrm{Pu}(\mathrm{IV})$ by two orders-ofmagnitude. The least pronounced effect of ligand loading was observed for $\mathrm{Eu}(\mathrm{III})$; the corresponding $K_{\mathrm{d}}$ values decreased only 1 order of magnitude when ligand loading increased from 2 to $47 \%$. Based on the similar behavior of Am(III) and Eu(III) seen in Figure 3.1, it can be expected that the loading effects for Am(III) would be similar to that for $\mathrm{Eu}(\mathrm{III})$. 


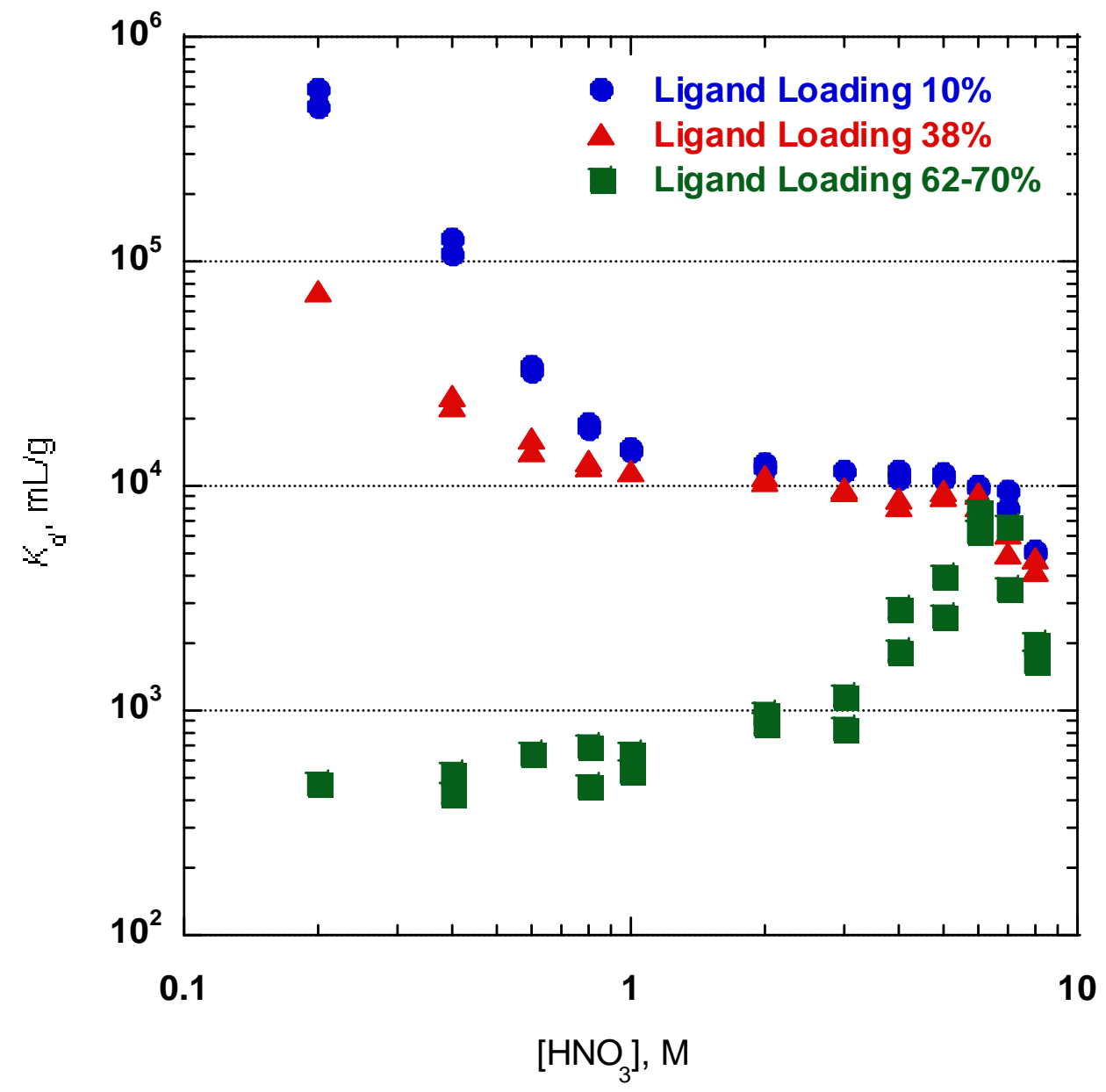

Figure 3.3. Acid Dependence of $\mathrm{Pu}(\mathrm{IV})$ Sorption at Variable ${ }^{\mathrm{n}} \mathrm{Pr}-\mathrm{Kläui}$ Resin Loading. $K_{\mathrm{d}}$ values were measured using $\mathrm{Pu}(\mathrm{IV})$ solutions of $2.13 \times 10^{-5} \mathrm{M}$ (blue circles), $8.50 \times 10^{-5} \mathrm{M}$ (red triangles), and $1.11 \times 10^{-4} \mathrm{M}$ (green squares) initial concentration as a function of $\mathrm{HNO}_{3}$ concentration. The resin weight was $\sim 30 \mathrm{mg}$ in each sample.

For $\mathrm{Pu}(\mathrm{IV})$, the loading experiment was additionally performed at $1 \mathrm{M} \mathrm{HNO}_{3}$. The sorption behavior of $\mathrm{Pu}(\mathrm{IV})$ at 0.3 and $1 \mathrm{M} \mathrm{HNO}_{3}$ was found to be drastically different (Figure 3.4), which confirmed our previous conclusion that the $\mathrm{Pu}(\mathrm{IV})$ sorption mechanism changed as the aqueous $\mathrm{HNO}_{3}$ concentration increased. Interestingly, no dependence of the corresponding $K_{\mathrm{d}}$ values on the ligand loading was observed until loading reached $50 \%$. 
- Eu(III), 0.3 M Nitric Acid

- $\mathrm{Pu}(\mathrm{IV}), 0.3 \mathrm{M}$ Nitric Acid

- Pu(IV), 1 M Nitric Acid

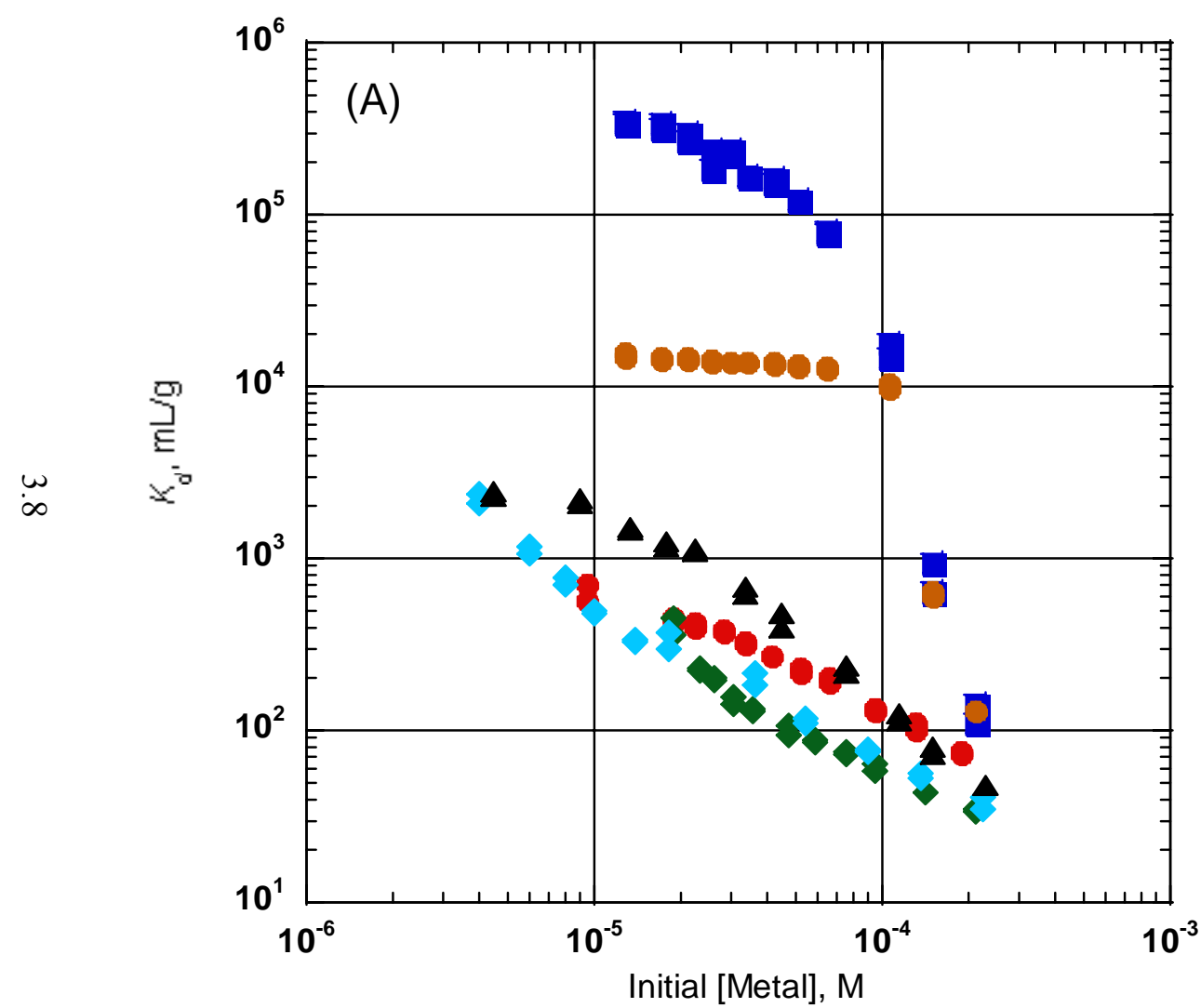

- $\mathrm{Np}(\mathrm{V}), 0.3 \mathrm{M}$ Nitric Acid

- Pu(VI), 0.3 M Nitric Acid

$\Delta \quad \mathrm{U}(\mathrm{VI}), 0.3 \mathrm{M}$ Nitric Acid

Figure 3.4. Effect of Initial Metal Ion Concentration (A) and ${ }^{\mathrm{n}} \mathrm{Pr}-\mathrm{Kläui}$ Resin Loading (B) on Actinide (III, IV, V, and VI) and Eu(III) Sorption.
$K_{\mathrm{d}}$ values were measured using aqueous solutions of variable metal ion concentration in $0.3 \mathrm{M} \mathrm{HNO}_{3}$. Sorbent weight was $\sim 30 \mathrm{mg}$ in

Figure 3.4. Effect of Initial Metal Ion Concentration (A) and ${ }^{\mathrm{n}} \mathrm{Pr}-\mathrm{Kläui}$ Resin Loading (B) on Actinide (III, IV, V, and VI) and Eu(III) Sorption.
$K_{\mathrm{d}}$ values were measured using aqueous solutions of variable metal ion concentration in $0.3 \mathrm{M} \mathrm{HNO}_{3}$. Sorbent weight was $\sim 30 \mathrm{mg}$ in each sample.

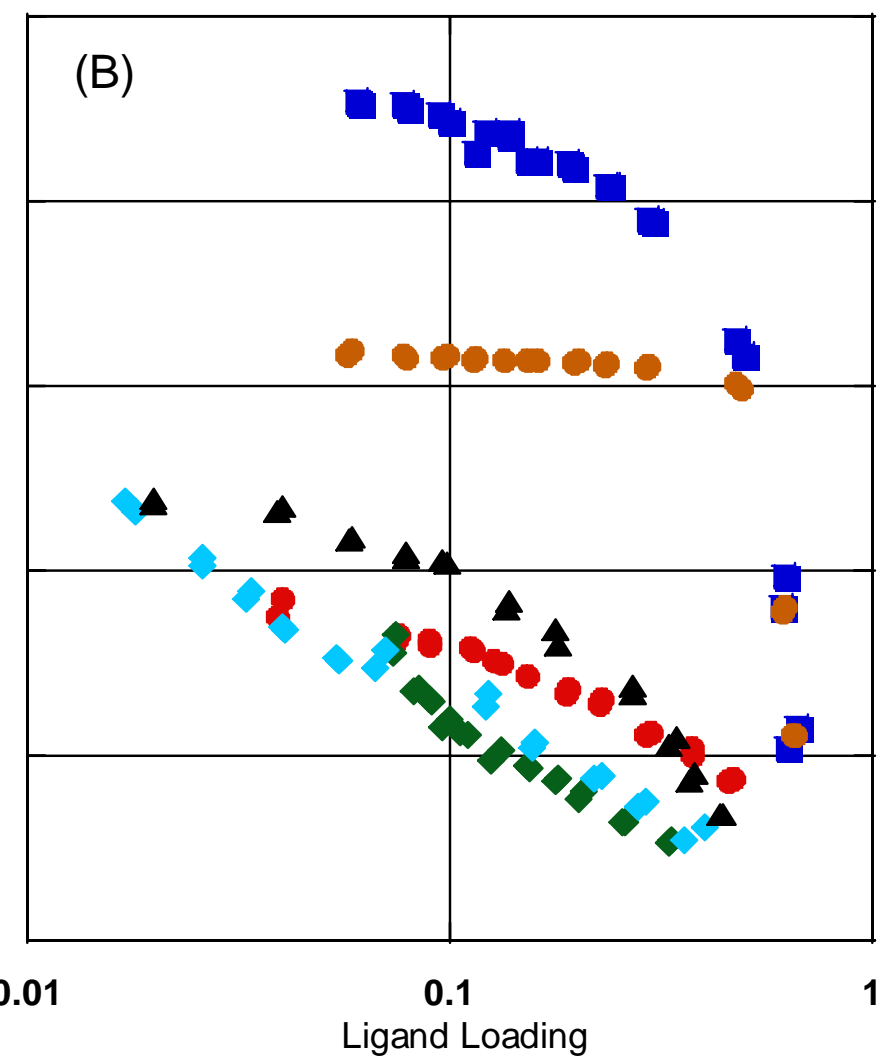




\subsection{Attempted Elution of Pu(IV) from Kläui Resins by Reducing to Pu(III)}

One of the challenges associated with applying the Kläui resins in automated radiochemical separations is the capability to elute the loaded $\mathrm{Pu}$ from the column for subsequent detection and quantification (Lumetta et al. 2006). Based on the batch $\mathrm{K}_{\mathrm{d}}$ values for Am(III) and Eu(III) (see Figure 3.1), it is conceivable that the $\mathrm{Pu}$ can be eluted by reducing the $\mathrm{Pu}(\mathrm{IV})$ to $\mathrm{Pu}(\mathrm{III})$ and eluting with $1 \mathrm{M}$ or higher $\mathrm{HNO}_{3}$ or $\mathrm{HCl}$. A previous attempt to reductively elute Pu from the Et-Kläui resin with iodide was unsuccessful. In this work, we attempted eluting Pu from the Kläui resins with ascorbic acid, hydroxylamine nitrate, and semicarbazide.

The elution of $\mathrm{Pu}$ with ascorbic acid was investigated with both the Et-Kläui resin and the ${ }^{\mathrm{n}} \mathrm{Pr}-\mathrm{Kläui}$ resin. A solution of $\mathrm{Pu}(\mathrm{IV})$ spiked into $4 \mathrm{M} \mathrm{HNO}_{3}(0.5 \mathrm{~mL})$ was passed through a column containing $\sim 0.2 \mathrm{~g}$ of either the Et-Kläui or the ${ }^{\mathrm{n}} \mathrm{Pr}-\mathrm{Kläui}$ resin. The column was washed with $4 \mathrm{M} \mathrm{HNO}_{3}$ followed by $0.1 \mathrm{M} \mathrm{HNO}_{3}$. The column was then eluted with $0.1 \mathrm{M}$ ascorbic acid in $1.5 \mathrm{M}$ formic acid and washed with water. All wash and eluant solutions were collected and weighed. The volume of each solution was determined from the mass and the density of the solution; the volumes collected are given in Table 3.1. The alpha activity in each solution was determined by LSC using Ultima-Gold XR as the scintillation cocktail.

Table 3.1 presents the results of this experiment. With the Et-Kläui resin, about 3\% of the Pu was not retained on the column and was thus found in the initial $4 \mathrm{M} \mathrm{HNO}_{3}$ washing solution. A slightly higher fraction $(\sim 10 \%)$ of the Pu was not retained by the ${ }^{\mathrm{n}} \mathrm{Pr}-\mathrm{Kläui}$ resin column. The amount of Pu eluted from either column with $0.1 \mathrm{M}$ ascorbic acid in $1.5 \mathrm{M}$ formic acid was insignificant. Subsequently, the columns were eluted with a solution consisting of $0.1 \mathrm{M}$ ascorbic acid/0.1 $\mathrm{M} \mathrm{H}_{2} \mathrm{SO}_{4} / 4 \mathrm{M} \mathrm{HNO}_{3}$. Again, very little $\mathrm{Pu}$ was removed from the column, although there was a slight elevation in the alpha activity of the eluant. The ${ }^{\mathrm{n}} \mathrm{Pr}-\mathrm{Kläui}$ resin column was subjected to two subsequent elution conditions. First, the elution with $0.1 \mathrm{M}$ ascorbic acid/ $0.1 \mathrm{M} \mathrm{H}_{2} \mathrm{SO}_{4} / 4 \mathrm{M} \mathrm{HNO}_{3}$ was repeated with the flow through the column stopped so that the eluant solution was in contact with the resin for $\sim 4 \mathrm{~h}$. Again, there was a slight rise in the alpha activity of the solution, but for the most part, the Pu remained on the column. Finally, the column was eluted with $0.1 \mathrm{M}$ hydroxylamine nitrate. No Pu was recovered from the column under these conditions.

In a separate experiment, $\mathrm{Pu}(\mathrm{IV})$ was loaded onto a 0.9 -g Et-Kläui resin from $0.5 \mathrm{~mL}$ of Pu-spiked $3 \mathrm{M} \mathrm{HNO}_{3}$. The column was washed with five $0.5-\mathrm{mL}$ portions of $4.8 \mathrm{M} \mathrm{HNO}_{3}$, with these portions being collected together in a single fraction along with the feed solution. The column was then eluted with $5 \mathrm{~mL} 0.01 \mathrm{M}$ semicarbazide hydrochloride followed by $2.5 \mathrm{~mL}$ of $0.1 \mathrm{M}$ semicarbizide in $0.05 \mathrm{M}$ $\mathrm{HNO}_{3}$. The column was washed with $3 \mathrm{~mL} 3 \mathrm{M} \mathrm{HNO}_{3}$ and then with $1 \mathrm{~mL}$ of water. The semicarbazide eluants and subsequent $\mathrm{HNO}_{3}$ and water washes were collected as a single fraction. The Pu behavior was determined by analyzing the original spiked solution and the fractions collected by LSC. Only $0.01 \%$ of the $\mathrm{Pu}$ was found in the semicarbazide eluant.

It can be concluded from the experiments described in the preceding paragraphs that elution of $\mathrm{Pu}(\mathrm{IV})$ from the Kläui resins by reduction to $\mathrm{Pu}(\mathrm{III})$ is difficult to achieve. It is likely that the high stability of the $\mathrm{Pu}(\mathrm{IV})-\mathrm{Kläui}$ complexes kinetically hinders the reduction of the $\mathrm{Pu}$. We shifted our efforts for recovery of $\mathrm{Pu}$ from the Kläui resin columns to elution of the ligand itself (along with the Pu complex) from the resin. This method is discussed in the following subsection. 
Table 3.1. Result of Ascorbic Acid and Hydroxylamine Nitrate Elution Experiments

\begin{tabular}{|c|c|c|c|c|c|c|}
\hline \multicolumn{7}{|c|}{ Et-Kläui Resin } \\
\hline Solution & Description & $\begin{array}{l}\text { Total Vol, } \\
\text { mL }\end{array}$ & $\begin{array}{l}\text { Alpha } \\
\text { cpm/mL }\end{array}$ & $\begin{array}{l}\text { Total Alpha, } \\
\text { cpm }\end{array}$ & $\begin{array}{l}\text { Pu Recovered, } \\
\%\end{array}$ & $\begin{array}{l}\text { Cumulative } \\
\text { Recovered, \% }\end{array}$ \\
\hline Feed & & 0.5 & 6556160 & 3278080 & & \\
\hline Et-Eluant-1 & $4 \mathrm{M} \mathrm{HNO}_{3}$ Wash & 4.66 & 18004 & 83899 & $2.6 \%$ & $2.6 \%$ \\
\hline Et-Eluant-2 & $\begin{array}{l}0.1 \mathrm{M} \text { ascorbic acid } / 1.5 \\
\mathrm{M} \text { formic acid }\end{array}$ & 4.43 & 13 & 57.59 & $0.0 \%$ & $2.6 \%$ \\
\hline Et-Eluant-3 & Water Wash & 4.47 & 4 & 17.88 & $0.0 \%$ & $2.6 \%$ \\
\hline Et-Eluant-4 & $\begin{array}{l}0.1 \mathrm{M} \text { ascorbic acid/0.1 } \\
\mathrm{M} \mathrm{H}_{2} \mathrm{SO}_{4} / 4 \mathrm{M} \mathrm{HNO}_{3} \\
\text { (includes } \sim 5 \mathrm{~mL} \\
\text { subsequent water wash) }\end{array}$ & 9.78 & 283 & 2768 & $0.1 \%$ & $2.7 \%$ \\
\hline \multirow{2}{*}{\multicolumn{7}{|c|}{${ }^{n}$ Pr-Kläui Resin }} \\
\hline & & & & & & \\
\hline Solution & Description & $\begin{array}{l}\text { Total Vol, } \\
\mathrm{mL}\end{array}$ & $\begin{array}{l}\text { Alpha } \\
\text { cpm/mL }\end{array}$ & $\begin{array}{l}\text { Total Alpha, } \\
\text { cpm }\end{array}$ & $\begin{array}{l}\text { Pu Recovered, } \\
\%\end{array}$ & $\begin{array}{l}\text { Cumulative } \\
\text { Recovered, \% }\end{array}$ \\
\hline Feed & & 0.5 & 6916040 & 3458020 & & \\
\hline Pr-Eluant-1 & $4 \mathrm{M} \mathrm{HNO}_{3}$ Wash & 5.75 & 61550 & 353913 & $10.2 \%$ & $10.2 \%$ \\
\hline Pr-Eluant-2 & $\begin{array}{l}0.1 \mathrm{M} \text { ascorbic acid } / 1.5 \\
\mathrm{M} \text { formic acid }\end{array}$ & 5.59 & 430 & 2404 & $0.1 \%$ & $10.3 \%$ \\
\hline Pr-Eluant-3 & Water Wash & 6.86 & 34 & 233 & $0.0 \%$ & $10.3 \%$ \\
\hline Pr-Eluant-4 & $\begin{array}{l}0.1 \mathrm{M} \text { ascorbic acid/0.1 } \\
\mathrm{M} \mathrm{H}_{2} \mathrm{SO}_{4} / 4 \mathrm{M} \mathrm{HNO}_{3} \\
\text { (includes } \sim 5 \mathrm{~mL} \\
\text { subsequent water wash) }\end{array}$ & 10.65 & 476 & 5069 & $0.1 \%$ & $10.4 \%$ \\
\hline Pr-Eluant-5 & $\begin{array}{l}0.1 \mathrm{M} \text { ascorbic acid/0.1 } \\
\mathrm{M} \mathrm{H}_{2} \mathrm{SO}_{4} / 4 \mathrm{M} \mathrm{HNO}_{3} \\
\text { with 4-h soak time } \\
\text { (includes } \sim 3 \mathrm{~mL} \\
\text { subsequent water wash) }\end{array}$ & 10.01 & 627 & 6276 & $0.2 \%$ & $10.6 \%$ \\
\hline
\end{tabular}

\subsection{Elution of Pu(IV) from Kläui Resins by Removing the Ligand from the Resin Support}

We have previously shown that Pu can be recovered from the Et-Kläui resin by dissolving the EtKläui ligand in an organic solvent and thereby washing it from the XAD-7 macroreticular support (Lumetta et al. 2004). When this is done, the Pu(IV)- Kläui complex is also washed from the XAD-7. However for subsequent processing and analysis of the $\mathrm{Pu}$ in the sample, the Kläui ligand must be destroyed. Two approaches to ligand destruction were investigated. The first was thermal destruction by heating to $400^{\circ} \mathrm{C}$. Although the ligand can be destroyed in this manner, subsequent dissolution of the 
residue and recovery of the $\mathrm{Pu}$ proved problematic, so thermal destruction was not further investigated. A more promising approach is to destroy the Kläui ligand by digestion in a mixture of three parts concentrated $\mathrm{HNO}_{3}$ and one part concentrated $\mathrm{HCl}$ ("reverse" aqua regia).

To investigate the recovery of Pu by chemical destruction of the Kläui ligand, a mock sample solution was prepared that was designed to mimic the solution generated by a lithium borate fusion of Rocky Flats soil (National Institute of Standards and Technology [NIST] standard reference material [SRM] 4353). This solution consisted of $0.071 \mathrm{M} \mathrm{Al}\left(\mathrm{NO}_{3}\right)_{3}, 0.016 \mathrm{M} \mathrm{Fe}\left(\mathrm{NO}_{3}\right)_{3}, 0.002 \mathrm{M} \mathrm{Ca}\left(\mathrm{NO}_{3}\right)_{2}, 0.007 \mathrm{M} \mathrm{NaNO}_{3}$, $0.93 \mathrm{M} \mathrm{LiNO}_{3}$, and $1 \mathrm{M} \mathrm{HNO}_{3}$. In this experiment, $2.01 \mathrm{~mL}$ of this solution was spiked with ${ }^{239} \mathrm{Pu}$. After removing a $10-\mu \mathrm{L}$ aliquot for $\mathrm{LSC}$, the spiked solution was divided into two $1-\mathrm{mL}$ portions (Feed \#1 and Feed \#2).

Feed \#1 was processed through an Et-Kläui resin column (0.303 $\mathrm{g}$ resin) in the following manner. The column was first pretreated with $0.5 \mathrm{~mL} 2.5 \mathrm{M} \mathrm{HNO}_{3}$. Feed \#1 was then loaded onto the column, and the column was washed with $3.0 \mathrm{~mL} 2.5 \mathrm{M} \mathrm{HNO}_{3}$ followed by $2.0 \mathrm{~mL}$ of water. All of these solutions were collected as a single fraction (1A). The ligand was washed from the column with $5.0 \mathrm{~mL}$ of $\mathrm{MeOH}$ with the resulting solution being collected as a single fraction (1B).

Feed \#2 was treated with $20 \mu \mathrm{L}$ of $6.12 \mathrm{M} \mathrm{NaNO}_{2}$ and allowed to stand under ambient conditions overnight. After this time, another $20 \mu \mathrm{L}$ of $6.12 \mathrm{M} \mathrm{NaNO}_{2}$ was added, and the mixture was heated at $\sim 100^{\circ} \mathrm{C}$ for $1.5 \mathrm{~h}$. This nitrite treatment was intended to make sure that all $\mathrm{Pu}$ was in the +4 oxidation state. The solution was processed through an Et-Kläui resin column $(0.301 \mathrm{~g}$ resin) in a manner exactly the same as was done for Feed \#1. The corresponding fractions collected from Feed \#2 were designated as $2 \mathrm{~A}$ (aqueous fraction) and $2 \mathrm{~B}(\mathrm{MeOH}$ fraction). The total alpha activity of fractions $1 \mathrm{~A}, 1 \mathrm{~B}, 2 \mathrm{~A}$, and 2B were determined by LSC (Table 3.2).

The unadjusted data in Table 3.2 indicate that the Pu mass recovery for Feed \#1 was $27 \%$ low whereas the Pu recovery for Feed \#2 was $27 \%$ high. This suggests that the spiked feed was not evenly divided into $1.0-\mathrm{mL}$ fractions at the start of the experiment. Assuming this to be true, the Pu distribution between the various fractions was recalculated (see Adjusted Results in Table 3.2). The adjusted results indicate that $97 \%$ of the $\mathrm{Pu}$ in Feed \#1 was retained on the column, but only $75 \%$ of the Pu in Feed \#2 was retained on the column. This result was somewhat surprising in that it appeared that the nitrite treatment actually decreased the fraction of $\mathrm{Pu}(\mathrm{IV})$ in the sample. One possible explanation for this is that nitrous acid was driven from the system when heated to $100^{\circ} \mathrm{C}$, and with nitrite ion, it depleted the heating step and caused some disproportionation of $\mathrm{Pu}(\mathrm{IV})$ to $\mathrm{Pu}(\mathrm{III})$ and $\mathrm{Pu}(\mathrm{VI})$ in this relatively low acid solution $\left(1 \mathrm{M} \mathrm{HNO}_{3}\right)$. Eliminating the heating step would likely solve this problem. 
Table 3.2. Results of Experiment to Recover Pu from Et-Kläui Resin with Methanol

\begin{tabular}{|c|c|c|c|c|c|}
\hline Solution $^{(a)}$ & $\begin{array}{l}\text { Total Vol, } \\
\text { mL }\end{array}$ & $\begin{array}{l}\text { Alpha } \\
\text { cpm/mL }\end{array}$ & $\begin{array}{l}\text { Total Alpha, } \\
\text { cpm }\end{array}$ & $\begin{array}{l}\text { Pu Recovered, } \\
\%\end{array}$ & $\begin{array}{l}\text { Cumulative } \\
\text { Recovered, \% }\end{array}$ \\
\hline Feed & 1 & 4586700 & 4586700 & N/A & $\mathrm{N} / \mathrm{A}$ \\
\hline \multicolumn{6}{|c|}{ Unadjusted Results } \\
\hline $1 \mathrm{~A}$ & 6.78 & 14142 & 95907 & $2.1 \%$ & $2.1 \%$ \\
\hline 1B & 5.36 & 606810 & 3254574 & $71.0 \%$ & $73.0 \%$ \\
\hline $2 \mathrm{~A}$ & 6.31 & 235110 & 1482536 & $32.3 \%$ & $32.3 \%$ \\
\hline $2 \mathrm{~B}$ & 5.63 & 773902 & 4359309 & $95.0 \%$ & $127.4 \%$ \\
\hline \multicolumn{6}{|c|}{ Adjusted Results } \\
\hline $1 \mathrm{~A}$ & 6.78 & 14142 & 95907 & $2.9 \%$ & $2.9 \%$ \\
\hline $1 \mathrm{~B}$ & 5.36 & 606810 & 3254574 & $97.1 \%$ & $100.0 \%$ \\
\hline $2 \mathrm{~A}$ & 6.31 & 235110 & 1482536 & $25.4 \%$ & $25.4 \%$ \\
\hline $2 \mathrm{~B}$ & 5.63 & 773902 & 4359309 & $74.6 \%$ & $100.0 \%$ \\
\hline
\end{tabular}

(a) See text for description of the various solutions analyzed.

To recover the $\mathrm{Pu}$ from the $\mathrm{MeOH}$ solution of the Et-Kläui ligand, the following strategy was investigated: 1) evaporate the $\mathrm{MeOH}, 2$ ) destroy the ligand in reverse aqua regia, 3) convert to $\mathrm{HNO}_{3}$ and treat with nitrite to convert to $\mathrm{Pu}(\mathrm{IV}), 4)$ separate $\mathrm{Pu}$ from $\mathrm{Co}$ and other components by anion exchange to provide a purified $\mathrm{Pu}$ fraction for analysis by thermal ionization mass spectrometry. Towards this end, fraction 1B was evaporated to dryness under a stream of air. The residue was dissolved in $1 \mathrm{~mL}$ concentrated $\mathrm{HNO}_{3}$, and then $0.25 \mathrm{~mL}$ concentrated $\mathrm{HCl}$ was added. The solution was heated at $100^{\circ} \mathrm{C}$ and allowed to evaporate. The resulting residue was taken up in $0.25 \mathrm{~mL}$ concentrated $\mathrm{HNO}_{3}$. After diluting with $0.75 \mathrm{~mL}$ water (to give a solution $\sim 4 \mathrm{M} \mathrm{HNO}_{3}$ ), the solution was allowed to cool to ambient temperature. The solution was diluted further with another $0.5 \mathrm{~mL}$ of water (to yield $\sim 2.6 \mathrm{M} \mathrm{HNO}_{3}$ ). To adjust the $\mathrm{Pu}$ oxidation state to $+4,0.1 \mathrm{~mL}$ of $6.12 \mathrm{M} \mathrm{NaNO}_{2}$ was added. Finally, $1.3 \mathrm{~mL}$ concentrated $\mathrm{HNO}_{3}$ was added to raise the $\mathrm{HNO}_{3}$ concentration to $7 \mathrm{M}$. The resulting solution was used as the feed to the anion exchange.

The anion exchange was performed using a gravity-fed column containing $0.52 \mathrm{~g}$ Dowex 1-X8. After first preconditioning the column with $0.5 \mathrm{~mL}_{7} \mathrm{M} \mathrm{HNO}_{3}$, the sample solution was fed through the column, followed by washing with $3 \mathrm{~mL} 7 \mathrm{M} \mathrm{HNO}_{3}$. These solutions were collected as a single fraction (1C). The column was eluted with $4 \mathrm{~mL} 1 \mathrm{M} \mathrm{HNO}_{3}, 1.5 \mathrm{~mL} 0.1 \mathrm{M} \mathrm{HNO}_{3}$, and $0.5 \mathrm{~mL}$ water (all of these were collected as fraction 1D). The column was washed with several additional milliliters of water ("Water Wash").

Table 3.3 presents the anion exchange results. About $20 \%$ of the Pu reported to fraction $1 \mathrm{C}$, suggesting incomplete conversion of $\mathrm{Pu}$ to $\mathrm{Pu}(\mathrm{IV})$ or incomplete destruction of the Kläui ligand. Only $25 \%$ more of the $\mathrm{Pu}$ was recovered in fraction $1 \mathrm{D}$ (where all the $\mathrm{Pu}$ was expected) and in the water wash. 
Thus, elution of the Pu from the Dowex 1-X8 column was surprisingly difficult. The column was further eluted with $5 \mathrm{~mL} 1.0 \mathrm{M} \mathrm{HCl}$ followed by $1 \mathrm{~mL}$ water (fraction $1 \mathrm{E}$ ) and then another $15 \mathrm{~mL} 1.0 \mathrm{M} \mathrm{HCl}$. The latter solution was combined with column wash water and evaporated before sampling for LSC (fraction $1 \mathrm{~F}$ ). Table 3.3 indicates eluting the column with $\mathrm{HCl}$ recovered additional $\mathrm{Pu}$, with the total recovery being $71 \%$.

Table 3.3. Results of Pu Ion Exchange Separation from Et-Kläui Residue

\begin{tabular}{|l|r|r|r|r|r|}
\hline & & \multicolumn{2}{|c|}{$\begin{array}{l}\text { Alpha } \\
\text { Solution }\end{array}{ }^{(a)}$} & Total Alpha, & \multicolumn{2}{l|}{$\begin{array}{l}\text { Pu Recovered, } \\
\text { \% }\end{array}$} & $\begin{array}{l}\text { Cumulative } \\
\text { Recovered, \% }\end{array}$ \\
\hline 1B (Recount) & 5.31 & 604230 & 3208461 & N/A & N/A \\
\hline 1C & 5.88 & 110324 & 649132 & $20.2 \%$ & $20.2 \%$ \\
\hline 1D & 6.09 & 117694 & 717062 & $22.3 \%$ & $42.6 \%$ \\
\hline Water Wash & 2.03 & 32610 & 66166 & $2.1 \%$ & $44.6 \%$ \\
\hline 1E & 6.01 & 109162 & 655721 & $20.4 \%$ & $65.1 \%$ \\
\hline 1F & 3.00 & 63291 & 189873 & $5.9 \%$ & $71.0 \%$ \\
\hline
\end{tabular}

(a) See text for description of the various solutions analyzed.

The results of the experiment with the mock dissolved Rocky Flats soil suggest that it is feasible to separate and recover Pu using the Et-Kläui resin, but some details of the chemistry still need to be worked out. In particular, the Pu valence adjustment following the ligand destruction step needs to be improved to allow complete conversion to the +4 valence state. Also, the elution of the anion exchange column needs improvement for complete recovery of the $\mathrm{Pu}$. This concept was further pursued using a solution prepared by acid digestion of Columbia River Sediment (NIST SRM 4350B).

The Columbia River Sediment was dissolved by microwave digestion in a mixture of nitric, hydrochloric, and hydrofluoric acids. The final acid concentrations were estimated to be $2.8 \mathrm{M} \mathrm{HNO}_{3}$, 3.2 $\mathrm{M} \mathrm{HCl}$, and 2.5 M HF. Boric acid was added to complex fluoride ion in the solution. A portion of the Columbia River Sediment solution $(10 \mathrm{~mL})$ was diluted with $10 \mathrm{~mL}$ of water and was spiked with $\mathrm{Pu}(\mathrm{IV})$. A column containing $0.53 \mathrm{~g}$ of the Et-Kläui resin was pretreated with $1 \mathrm{~mL} 2.5 \mathrm{M} \mathrm{HNO}_{3}$, and then the 20-mL sample was passed though the column. The effluent was collected in nominally 5-mL fractions (fractions 1 through 4). Once all the sample had been run through the column, the column was washed with $5 \mathrm{~mL} 2.5 \mathrm{M} \mathrm{HNO}_{3}$ and $1 \mathrm{~mL}$ water, which were also collected into fraction 4 . Finally, the column was washed with $2 \mathrm{~mL}$ water and $6 \mathrm{~mL}$ methanol, with these being collected together as fraction 5 .

Table 3.4 presents the results of the Et-Kläui column run with the dissolved Columbia River Sediment sample. The total Pu mass recovery was $88 \%$ in this column experiment. The column on the far right of the table gives the fraction of $\mathrm{Pu}$ in each fraction, relative to the total amount of $\mathrm{Pu}$ recovered in the experiment (i.e., the sum of the alpha activity in fractions 1 through 5 ). On this basis, $\sim 50 \%$ of the $\mathrm{Pu}$ in the Columbia River Sediment sample was retained on the column. A similar experiment with the same amount of sample solution, but less resin on the column $(0.11 \mathrm{~g}$ versus $0.53 \mathrm{~g})$ resulted in only $\sim 20 \%$ retention of $\mathrm{Pu}$ on the column. This can be explained simply in terms of the Kläui ligand available for binding to $\mathrm{Pu}(\mathrm{IV})$. For the column containing $0.11 \mathrm{~g}$ of resin, the estimated Kläui ligand-to-plutonium 
ratio was 2.7, which is only slightly above a stoichiometric amount for formation of the complex $\left[\mathrm{Pu}\left(\mathrm{NO}_{3}\right)_{3} \mathrm{~L}_{2}\right](\mathrm{L}=$ the Et- Kläui ligand $1 \mathrm{~b})$. The amount of ligand available can be increased by increasing the amount of resin (as was done here - the ligand-to-plutonium ratio was increased to 15 in the experiment with $0.53 \mathrm{~g}$ of resin) or by increasing the loading of the Kläui ligand on the inert resin support.

The Columbia River Sediment sample contains large amount of iron ( $6.8 \mathrm{wt} \% \mathrm{Fe})$. We have previously demonstrated that $\mathrm{Fe}$ (III) suppresses the sorption of $\mathrm{Pu}(\mathrm{IV})$ by the Et-Kläui resin (Lumetta et al. 2004). It was calculated that $\mathrm{Fe} / \mathrm{Kläui}$ ligand/Pu molar ratio in the elution system was $60 / 1 / 0.07$ for the experiment described in Table 3.4. This corresponds to about 860 molar excess of Fe over Pu. Based on this result, it was concluded that $\mathrm{Pu} / \mathrm{Fe}$ selectivity of the tested Kläui ligand is high but improvements in the $\mathrm{Pu} / \mathrm{Fe}$ selectivity would yield a more robust separations media for the processing of the soil samples containing high Fe levels (see Section 4 for a description of on-going efforts to improve the selectivity for $\mathrm{Pu}$ over $\mathrm{Fe}$ ). This approach needs refinement, but this experiment does show the potential for the Kläui resin for selectively separating $\mathrm{Pu}$ from complex sample matrices. The capability to directly separate $\mathrm{Pu}$ from such matrices would be significant because it would eliminate the need for precipitation steps, which are difficult to automate.

Fraction 5 was evaporated, and the residue was digested at $100^{\circ} \mathrm{C}$ in $1.5 \mathrm{~mL}$ concentrated $\mathrm{HNO}_{3}$ plus $0.5 \mathrm{~mL}$ concentrated $\mathrm{HCl}$. The acids were allowed to evaporate under these conditions. The residue was dissolved in $1 \mathrm{~mL}$ concentrated $\mathrm{HNO}_{3}$. The resulting solution was diluted to $4 \mathrm{M} \mathrm{HNO}_{3}$ by adding $2.9 \mathrm{~mL}$ water. The solution was treated with two $0.2-\mathrm{mL}$ portions of $\mathrm{NaNO}_{2}$ to convert $\mathrm{Pu}$ to the +4 oxidation state. After standing for about 10 minutes, $2.5 \mathrm{~mL}$ of concentrated $\mathrm{HNO}_{3}$ was added, yielding a clear solution (with $\mathrm{NO}_{\mathrm{x}}$ evolution).

An anion exchange column was prepared with $0.75 \mathrm{~g}$ of Bio-Rad AG 1-X4 (100-200 mesh) that had been previously washed to remove fines. The column was pretreated with $1 \mathrm{~mL}$ of $7 \mathrm{M} \mathrm{HNO}_{3}$, and then the solution described in the previous paragraph was passed through the column. These solutions were collected as a single fraction (fraction 6). The column was washed with $5 \mathrm{~mL} 7 \mathrm{M} \mathrm{HNO}_{3}$ (fraction 7), eluted with $6 \mathrm{~mL} 1 \mathrm{M} \mathrm{HCl}$, and washed with $4 \mathrm{~mL}$ water (the $\mathrm{HCl}$ and water were collected together as fraction 8). Table 3.4 presents the results of this anion exchange experiment. As previously observed, the mass recovery of $\mathrm{Pu}$ from the ion exchange column was low (56\%). However, in this case, it appeared that the $\mathrm{Pu}$ valence adjustment was much more successful with only $2 \%$ of the Pu being found in fractions 6 and 7.

\subsection{Batch Equilibrium Distribution Experiments with the SAMMS Materials}

Recently synthesized high-performance SAMMS sorbents were evaluated for their potential ability to collect and pre-concentrate radionuclides relevant to special nuclear material (SNM) production. The specific actinide ions investigated were Am(III), Th(IV), $\mathrm{Pu}(\mathrm{IV}), \mathrm{Np}(\mathrm{V})$, and U(VI). SAMMS have been developed at PNNL as a powerful new class of sorbent materials with unprecedented capabilities. The high surface area contained in a very small volume gives rise to high sorption capacity, and the rigid open pore structure provides for rapid sorption kinetics. Furthermore, the SAMMS surface chemistry can be easily tailored for a wide variety of target ions. The assembly process for SAMMS is shown in Figure 3.5. These materials have been shown to be excellent sorbents because they are capable of efficiently and selectively sequestering volatile organics, metals, and radionuclides from complex matrices (Lin et al. 2005; Fryxell et al. 2005; Yantasee et al. 2003; Birnbaum et al. 2002; Lin et al. 2001). 
The efficiency, stability, capacity, and versatility of the SAMMS materials make them a superior foundation upon which to develop advanced chemical separation technologies.
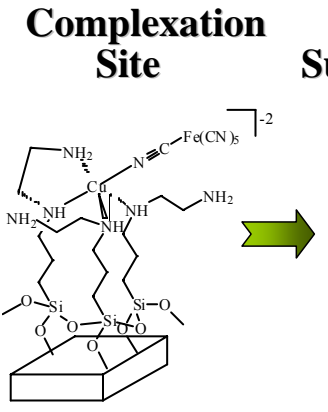

Surface Ligands

-High Affinity

-High Selectivity

-Catalytic
Polyfunctional Surface Monolayer
High Density Surface Layer

1-6 binding sites/ $\mathrm{nm}^{2}$ -High capacity

-Chemically selective

-Flexible chemistry
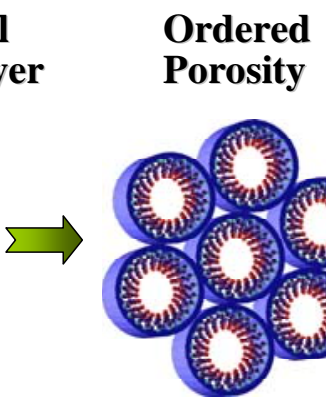
$\underline{\text { High surface area }}$ $\left(400-1400 \mathrm{~m}^{2} / \mathrm{g}\right)$
- Sensitivity
- Capacity

Nondendritic porosity

- rapid response

- $\quad$ size selective

\section{Macroscopic} Support

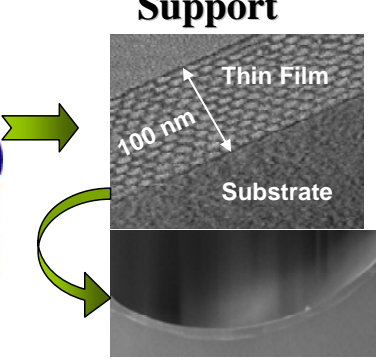

Variable Form Factor -Thin films

- Shown above is a 1 micron mesoporous thin film within

-Particles a 75 micron capillary

-Monoliths

Figure 3.5. A Graphical Depiction of the Construction of SAMMS. SAMMS integrate high surface area nanomaterials with a rigid open pore structure and high-density surface chemistry easily tailored for a wide variety of target compounds. This gives SAMMS materials excellent performance characteristics as selective sorbent/chromatorgraphic/preconcentrator materials when engineered for specific applications. 
Table 3.4. Results of Experiment to Recover Pu from Dissolved Columbia River Sediment

\begin{tabular}{|c|c|c|c|c|c|c|}
\hline Solution & Total Vol, $\mathrm{mL}$ & Alpha cpm/mL & $\begin{array}{l}\text { Total Alpha, } \\
\text { cpm }\end{array}$ & $\begin{array}{l}\text { Pu } \\
\text { Recovered, } \\
\%\end{array}$ & $\begin{array}{l}\text { Cumulative } \\
\text { Recovered, \% }\end{array}$ & $\begin{array}{l}\text { Pu Relative } \\
\text { to Total } \\
\text { Recovered, } \\
\%\end{array}$ \\
\hline Spiked Sample & 19.9 & 802704 & 15973810 & $\mathrm{~N} / \mathrm{A}$ & $\mathrm{N} / \mathrm{A}$ & \\
\hline Fraction- 1 & 6.13 & 46664 & 286235 & $1.8 \%$ & $1.8 \%$ & $2.0 \%$ \\
\hline Fraction-2 & 4.99 & 232298 & 1159378 & $7.3 \%$ & $9.0 \%$ & $8.3 \%$ \\
\hline Fraction-3 & 6.51 & 460236 & 2998228 & $18.8 \%$ & $27.8 \%$ & $21.4 \%$ \\
\hline Fraction-4 & 8.99 & 299518 & 2691225 & $16.8 \%$ & $44.7 \%$ & $19.2 \%$ \\
\hline Fraction-5 & 8.48 & 810784 & 6878151 & $43.1 \%$ & $87.7 \%$ & $49.1 \%$ \\
\hline \multicolumn{2}{|c|}{ Amount $\mathrm{Pu}$ into IX process } & & 6837612 & & & \\
\hline Fraction- 6 & 8.08 & 2984 & 24101 & $0.4 \%$ & $0.4 \%$ & \\
\hline Fraction-7 & 5.53 & 15028 & 83177 & $1.2 \%$ & $1.6 \%$ & \\
\hline Fraction- 8 & 11.84 & 316076 & 3743346 & $54.7 \%$ & $56.3 \%$ & \\
\hline
\end{tabular}


Batch distribution coefficients from nitric acid and hydrochloric acid solutions were determined for Am(III), Th(IV), Pu(IV), Np(V), and U(VI) using three different SAMMS materials. Figure 3.6

illustrates the results of these measurements. The sorption behavior is essentially independent of whether $\mathrm{HNO}_{3}$ or $\mathrm{HCl}$ is used. In many respects, the behavior of the diphosphonate-derivatized SAMMS materials is similar to that for the Kläui resin. Very strong absorption of the tetravalent actinides is observed throughout the range of acid concentrations examined $(0.01$ to $3 \mathrm{M})$. Depending on the acid concentration, the sorption of $\mathrm{U}(\mathrm{VI})$ is two to three orders-of-magnitude less than that for tetravalent $\mathrm{Pu}$ or Th. The trend for Am(III) is very similar to that for the Kläui resin in that fairly strong absorption occurs at low acid concentration, but the $\mathrm{Am} \mathrm{K}_{\mathrm{d}}$ decreases steadily with increasing acid concentration. The sorption of $\mathrm{Np}(\mathrm{V})$ is relatively low, but is largely independent of the acid concentration.

Because of the high affinity of the SAMMS materials for Pu(IV), the elution of this ion might be an issue (as has been the case for the Kläui resin). The elution of $\mathrm{Pu}$ by reduction to $\mathrm{Pu}$ (III) needs to be explored. Should this fail, alternative elution methods, such as cleavage of the silane from the silica support with aggressive acidic oxidizing conditions or dissolution of entire sorbent material with HF and nitric acid, could also be considered. 

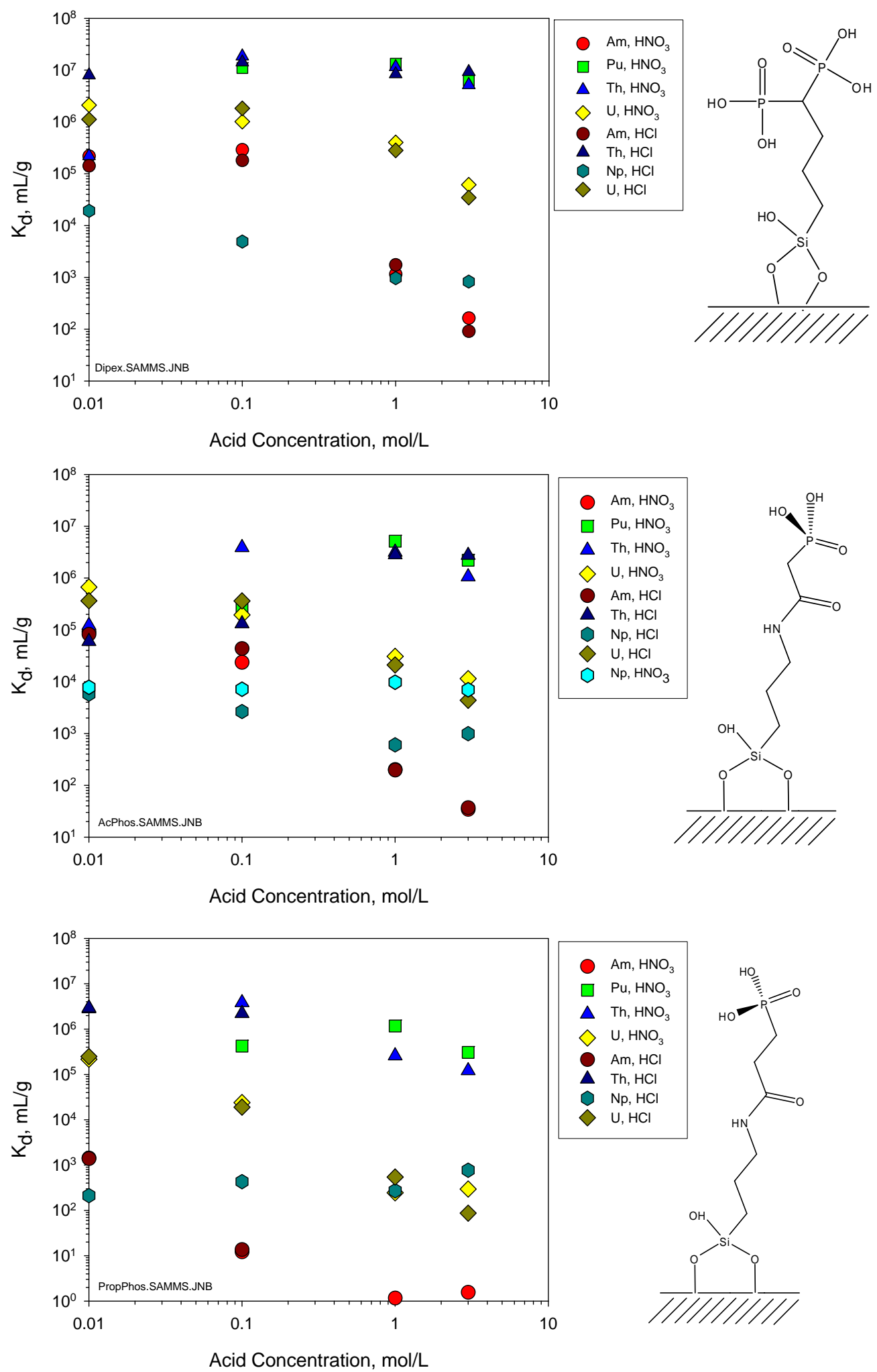

Figure 3.6. Batch Distribution Coefficients for Actinide Ions on Three Different SAMMS Materials; the actinide oxidation states are $\mathrm{Am}(\mathrm{III}), \mathrm{Pu}(\mathrm{IV}), \mathrm{Th}(\mathrm{IV}), \mathrm{Np}(\mathrm{V})$, and $\mathrm{U}(\mathrm{VI})$ 


\subsection{Computational Ligand Design}

As discussed previously in this report, the high affinity of Kläui ligands for tetravalent actinides offers the possibility that Kläui-based resins could be used to extract and concentrate trace actinides from aqueous solution. Furthermore, it can be envisioned that Kläui ligands deployed in a "dipstick" configuration (i.e., ligand bound to a surface that can be directly dipped into the analyte solution) could be used to collect tetravalent actinides from a variety of samples for subsequent radiochemical detection. In such applications, improved performance would be obtained by 1) increasing the actinide affinity of the ligand and 2) suppressing interferences caused by transition metal ions. As discussed below, there is reason to believe that these objectives can be met by constructing a single sequestering agent from two Kläui ligand components.

\subsection{Structural Design Basis}

Kläui ligands bind to most metal ions through the three $\mathrm{P}=\mathrm{O}$ oxygen atoms, forming a tripodal structure as shown in Figure 4.1.
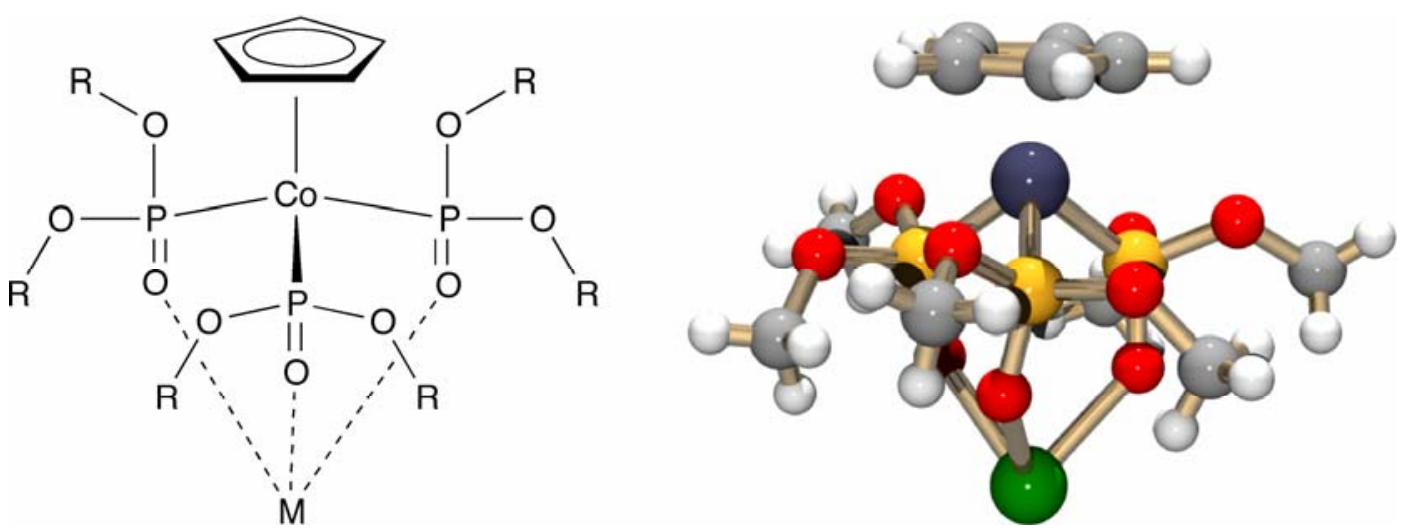

Figure 4.1. Illustration Showing How the Kläui Ligand Interacts with a Metal Ion. The ball and stick representation on the right is a fragment taken from an actual crystal structure.

It is known from crystal-structure data that two Kläui ligands can bind to one metal ion at the same time. For smaller metal ions, this yields an octahedral arrangement of oxygen atoms in the inner sphere, coordinatively saturating the metal. With larger metal ions, such as the tetravalent and trivalent actinides, two Kläui ligands do not provide a sufficient number of donor groups to fill the coordination sphere, and additional ligands are required. As illustrated in Figure 4.2 and Figure 4.3, the spatial orientation of one Kläui ligand with respect to the other is dictated by the size of the metal ion. Whereas in the octahedral complex, each triad of ligating oxygen atoms is parallel to the others; in an eight-coordinate square antiprism complex, one triad is tilted relative to the others. 


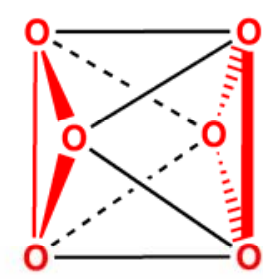

octahedron

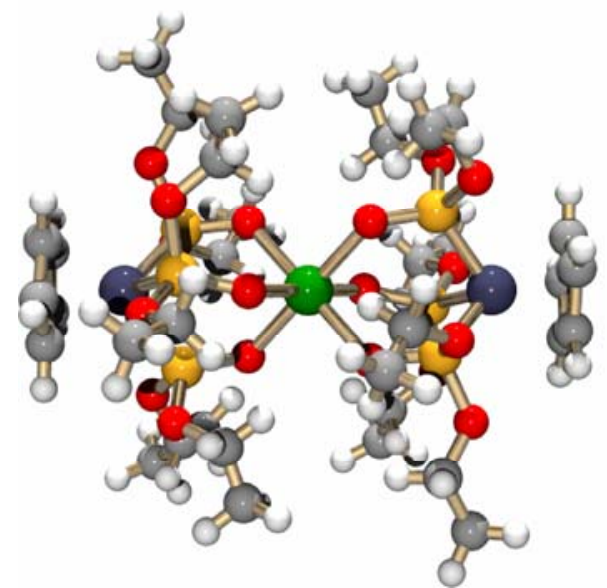

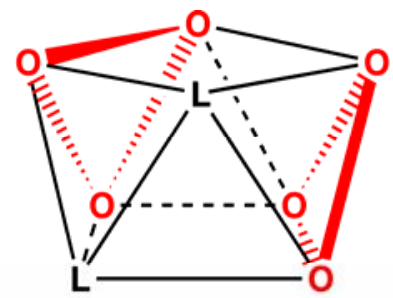

square antiprism

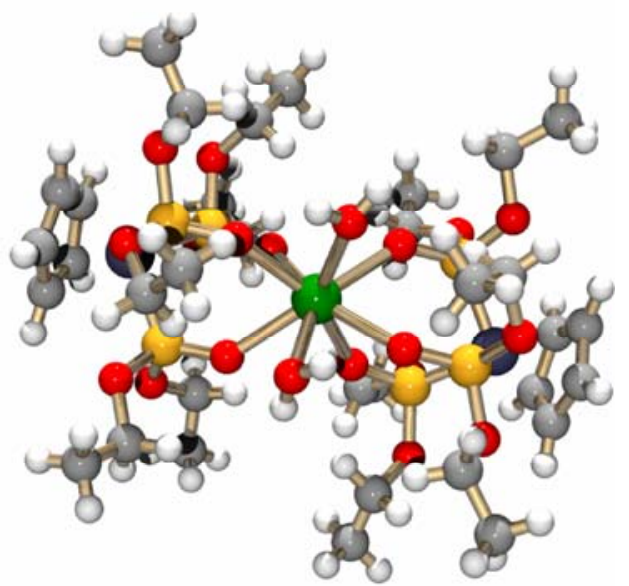

Figure 4.2. Crystal Structures of Six-Coordinate $\mathrm{Mn}^{3+}$ (left) and Eight-Coordinate $\mathrm{Eu}^{3+}$ (right) Complexes Containing Two Kläui Ligands. In the six-coordinate structure, the Kläui ligands occupy opposing triangular faces of an octahedron. In the eight-coordinate structure, the Kläui ligands occupy triangular faces spanning the square faces of a distorted square antiprism. In the latter example, vacant coordination sites are occupied by water ligands.

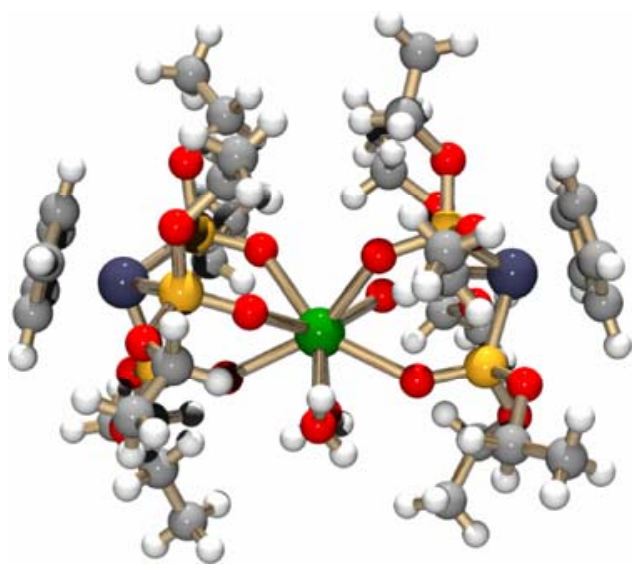

Figure 4.3. Alternate View of the $\mathrm{Eu}^{3+}$ Complex (Figure 4.2, right) 
Connecting two Kläui ligands together to form a single hexadentate ligand can, in principle, enhance both metal ion selectivity and binding affinity. Enhanced selectivity for larger metal ions will be achieved when the ligand structure constrains the two Kläui tripods to adopt a geometry associated with large metals, but prevents them from adopting the geometry associated with smaller metal ions. Achieving this constraint will likely require a fairly rigid linkage between the two Kläui binding sites. One way that this might be achieved is by phosphonate ester linkages to a connecting scaffold as shown in Figure 4.4.

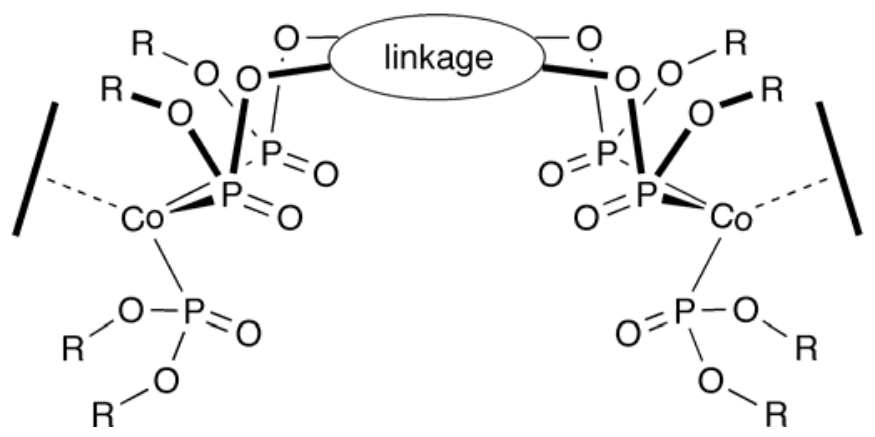

Figure 4.4. A Conceptual Hexadentate Ligand Formed by Linking Two Kläui Ligands to a Scaffold via Phosphonate Ester Bonds

Enhanced binding affinity will result from the entropic consequences of tying the two Kläui ligands together. This phenomenon, known as the chelate effect (Steed 2000), should increase the free energy of binding over that obtained with two isolated Kläui ligands. The magnitude of this effect has been estimated to be $2.4 \mathrm{kcal} / \mathrm{mol}$ (an increase of 55.6 in binding constant) (Schneider and Yatsimirsky 2000). The maximum increase will be attained when the ligand is pre-organized for binding, in other words, when the metal-binding conformation of the ligand is the only significantly populated conformation in solution.

\subsection{Approach}

The preceding section defined a goal for the initial design effort - to identify potential linking structures that constrain two Kläui ligands in space such that 1) they both achieve an optimal interaction with a large metal ion (e.g., $\mathrm{Pu}^{4+}$ ), and 2) only one of them can achieve a good interaction with a small metal ion (e.g., $\mathrm{Fe}^{3+}$ ). This will lead to a situation in which the chelate effect can be engaged for $\mathrm{Pu}^{4+}$ ion but not for $\mathrm{Fe}^{3+}$ ion, leading to high selectivity for $\mathrm{Pu}^{4+}$ over $\mathrm{Fe}^{3+}$.

To achieve this goal, we intend to use our in-house de novo design software, HostDesigner (Hay and Firman 2002; Hay et al. 2005; Bryantsev and Hay 2006) to search for appropriate linkages. The approach requires knowledge of the 3-D structure of the Kläui ligand and its metal complexes, which will be used to construct input files for this search. In addition, evaluating potential linkages requires a working molecular mechanics model that can treat these systems. For this purpose, we will extend the default MM3 parameter set of commercial molecular mechanics software, PCModel (Gilbert 2006).

The approach involves the following steps:

(1) Mine the Cambridge Crystal Structural Database to obtain geometric parameters for the Kläui ligand and its metal complexes 
(2) Develop a set of molecular mechanics model (MM3) parameters for the Kläui ligand by fitting to crystal structure data

(3) Extend this parameter set to include Kläui ligand metal complexes by fitting to crystal structure data and through the use of empirical relationships

(4) Predict structures for $\left[\mathrm{Pu}(\mathrm{Kläui})_{2}\left(\mathrm{OH}_{2}\right)\right]^{2+}$ complexes for use as input to HostDesigner

(5) Identify candidate linking structures with HostDesigner

(6) Evaluate and rank the resulting bis-Kläui ligands with the extended MM3 model.

\subsection{Results}

\subsubsection{Analysis of Crystal Structure Data for Kläui Metal Complexes}

The Cambridge Structure Database (CSD) (Allen 1993) was searched for all structures containing the Kläui ligand, yielding 117 examples. By visual inspection, the majority of these structures could be distributed into 5 classes as shown in Figure 4.5. These include 1) simple 1:1 Kläui-metal complexes in which the coordination sphere is completed by other ligands (61 examples), 2) 2:1 Kläui-metal complexes where there are no other ligands present (8 examples), 3) 2:1 Kläui-metal complexes where there are additional ligands in the metal coordination sphere (five examples), 4) two 1:1 Kläui-metal complexes

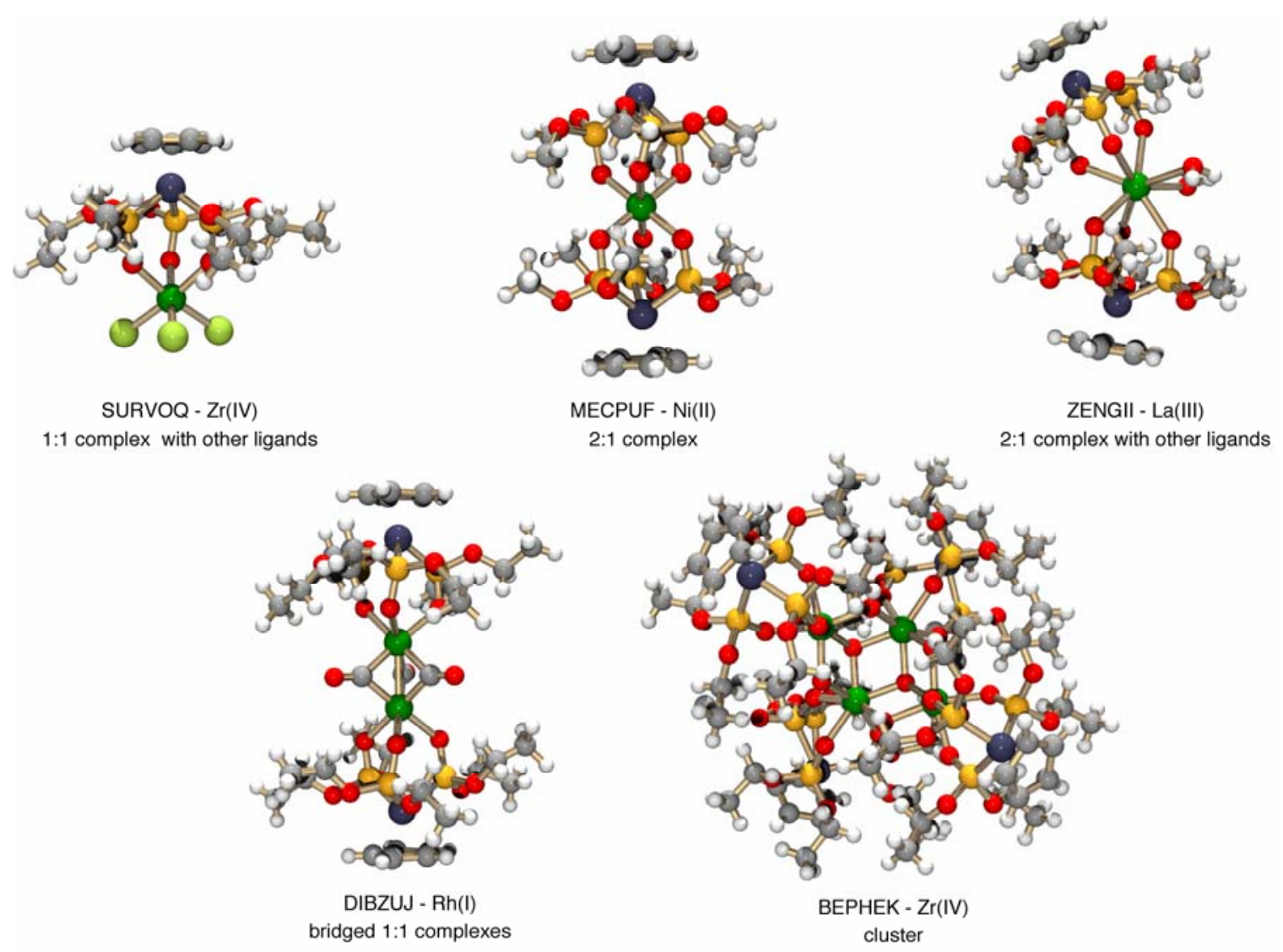

Figure 4.5. Examples of the Types of Kläui-Metal Complex Structures Available in the CSD. The six letter CSD reference code and chelated metal ion are indicated for each structure. 
in which the two metals were bridged by other ligands (26 examples), and 5) clusters formed by combining three or more Kläui ligands with three or more metals (8 examples).

Such crystal structure data are useful for two reasons. First, they provide a description of the threedimensional structure of the Kläui ligand. An analysis of selected geometric parameters obtained from these data is presented in the remainder of this section. Second, they provide a set of test structures on which to develop and validate a molecular mechanics model. This activity is described in further detail in the next section (see Section 4.3.2).

Data for selected bond distances and bond angles in the Kläui ligand were collected during the CSD search and plotted using the Vista software utility that is part of the CSD system. Statistical analysis of these data yielded the mean values and standard deviations presented in Table 4.1. Histograms showing the distribution of data that yielded the values given in Table 4.1 are presented below in Figure 4.6 and Figure 4.8.

Similar data also were collected for geometric parameters that describe the interaction of a metal ion, $\mathrm{M}$, with the Kläui ligand. A wide variety of metal ions is represented in the 117 crystal structures. As a result, the $\mathrm{M}-\mathrm{O}$ distances vary from 1.88 to $2.70 \AA$. Histograms of $\mathrm{P}=\mathrm{O}-\mathrm{M}$ angles (Figure 4.7, bottom right) as well as $\mathrm{O}-\mathrm{P}=\mathrm{O}-\mathrm{M}$ and $\mathrm{Co}-\mathrm{P}=\mathrm{O}-\mathrm{M}$ dihedral angles (Figure 4.8) provide insight regarding any directionality that might be present at the oxygen donor atoms. The $\mathrm{P}=\mathrm{O}-\mathrm{M}$ angle distribution establishes that the majority of the data ranges between 120 and $140^{\circ}$. The dihedral angle plots reveal a preference for 0 and $\pm 120^{\circ}$, showing a marked preference for the $\mathrm{M}-\mathrm{O}$ bond to eclipse the $\mathrm{P}-\mathrm{Co}$ bond.

Table 4.1. Mean Bond Distances $(\AA)$ and Angles $\left(^{\circ}\right)$ for the Kläui Ligand

\begin{tabular}{lcc}
\hline Parameter & Mean & Standard Deviation \\
\hline Co-C & 2.074 & 0.024 \\
$\mathrm{C}-\mathrm{C}$ & 1.392 & 0.041 \\
$\mathrm{P}-\mathrm{Co}$ & 2.161 & 0.022 \\
$\mathrm{P}-\mathrm{O}$ & 1.599 & 0.040 \\
$\mathrm{P}=\mathrm{O}$ & 1.510 & 0.026 \\
$\mathrm{C}-\mathrm{O}$ & 1.413 & 0.067 \\
$\mathrm{C}-\mathrm{C}-\mathrm{C}$ & 108.0 & 2.5 \\
$\mathrm{P}-\mathrm{Co}-\mathrm{P}$ & 91.0 & 1.6 \\
$\mathrm{Co}-\mathrm{P}-\mathrm{O}$ & 109.1 & 3.5 \\
$\mathrm{Co}-\mathrm{P}=\mathrm{O}$ & 118.9 & 2.0 \\
$\mathrm{O}-\mathrm{P}-\mathrm{O}$ & 101.3 & 4.1 \\
$\mathrm{O}-\mathrm{P}=\mathrm{O}$ & 108.2 & 2.4 \\
$\mathrm{P}-\mathrm{O}-\mathrm{C}$ & 123.0 & 4.2 \\
$\mathrm{P}=\mathrm{O}-\mathrm{M}$ & 131.3 & 5.1 \\
\hline
\end{tabular}



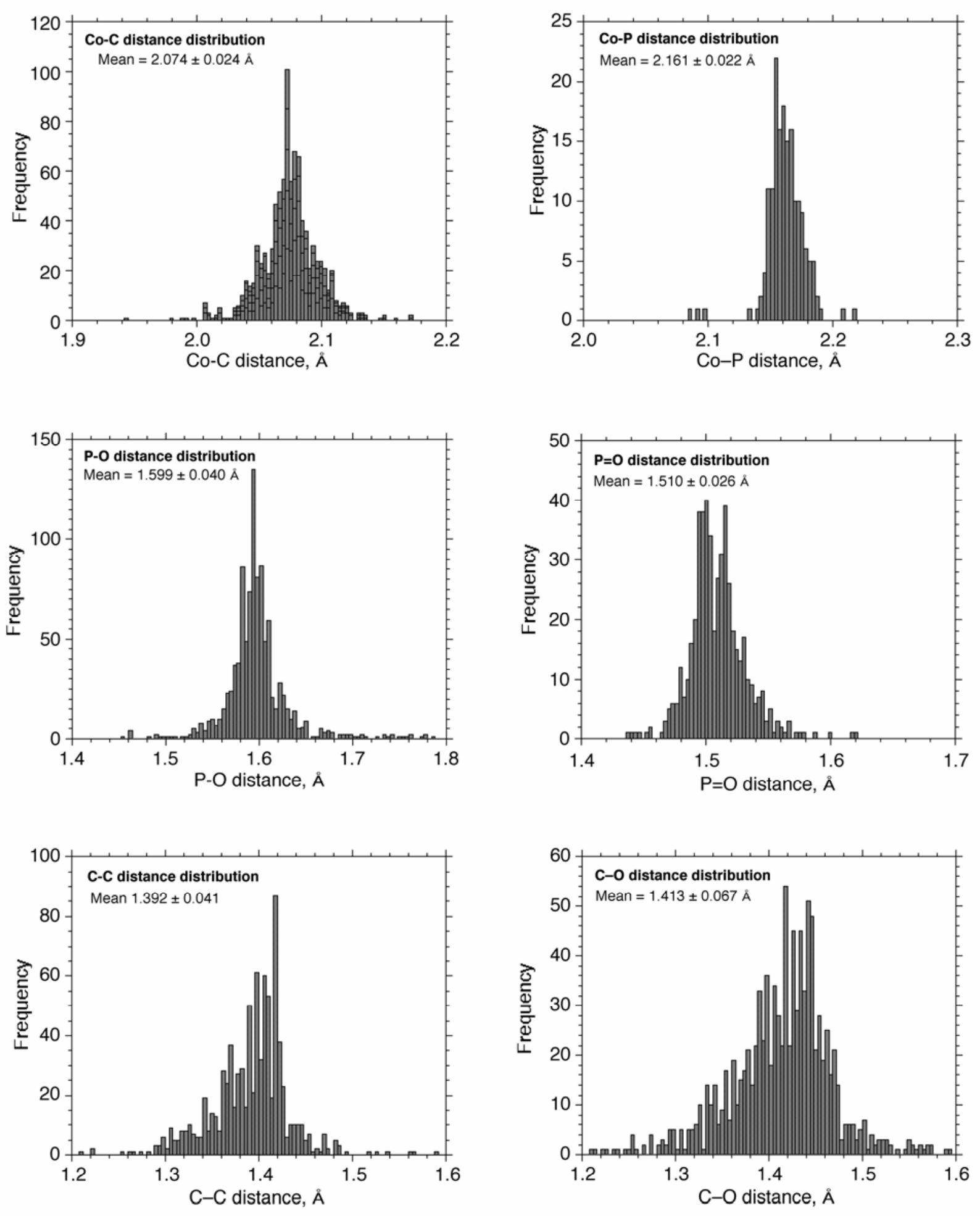

Figure 4.6. Histograms of Bond Distances Observed in Kläui Ligand Crystal Structures 

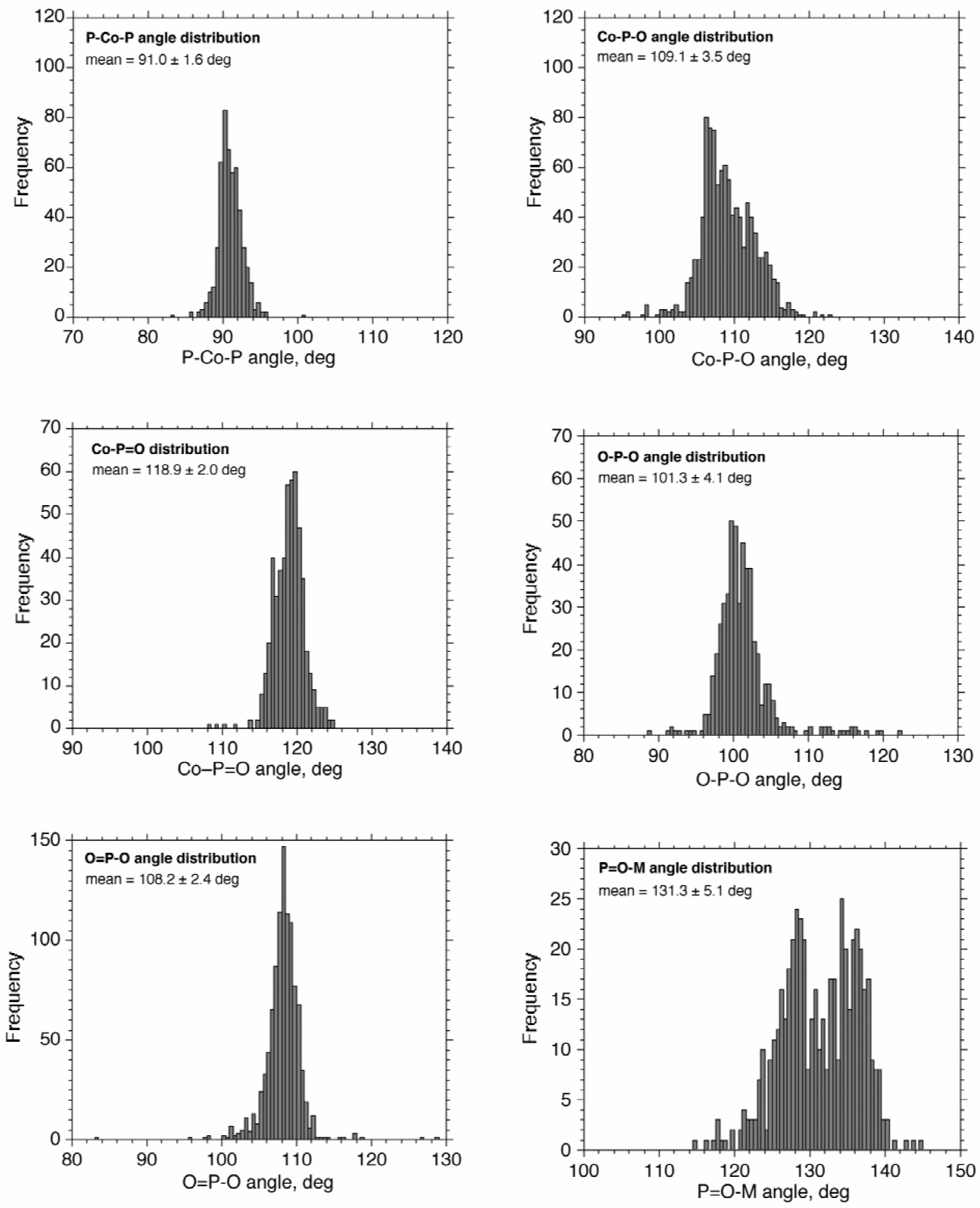

Figure 4.7. Histograms of Angles Observed in Kläui Ligand Crystal Structures 

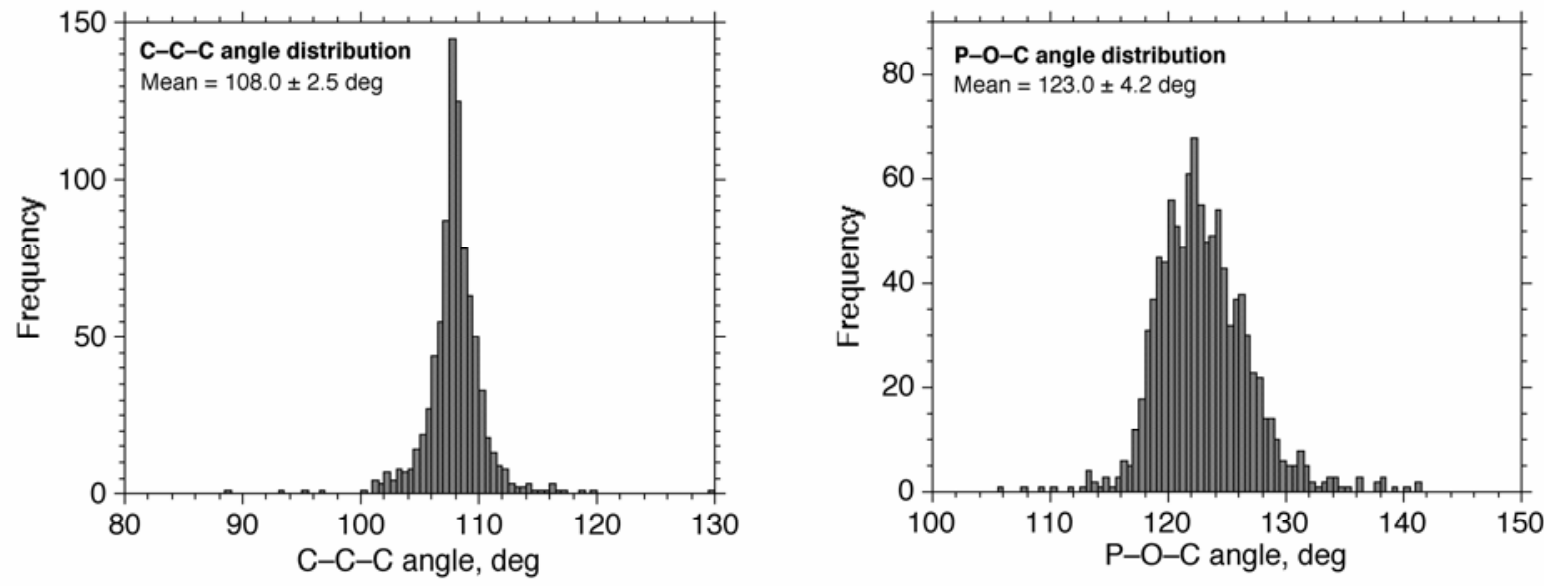

Figure 4.7 (Contd)
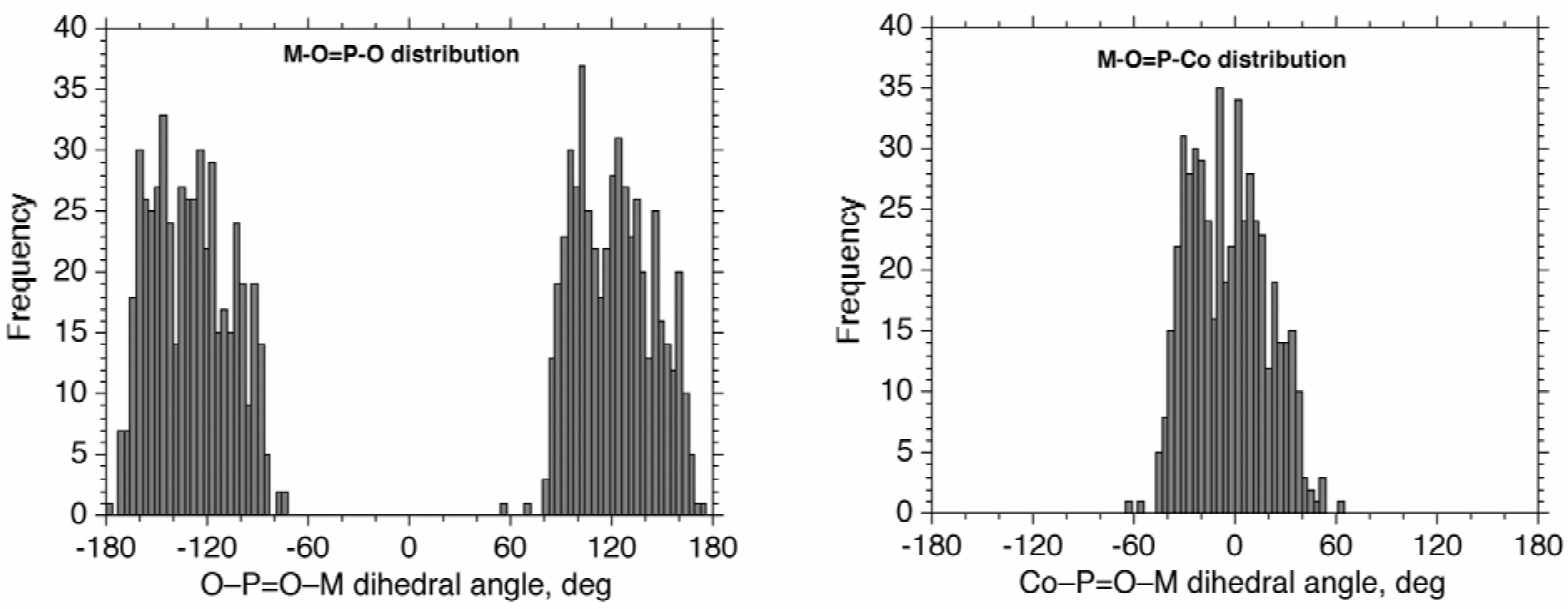

Figure 4.8. Histograms of Dihedral Angles Observed in Kläui Ligand Crystal Structures

\subsubsection{Extension of MM3 to Treat Kläui Ligands and their Metal Complexes}

Historically, molecular mechanics models have been developed for exclusive application to organic molecules, in other words, molecules composed of $\mathrm{C}, \mathrm{H}, \mathrm{N}, \mathrm{P}, \mathrm{O}, \mathrm{S}, \mathrm{F}, \mathrm{Cl}, \mathrm{Br}$, and I atoms (Burkert and Allinger 1982). All commercial molecular mechanics software packages come with sets of parameters for organic compounds, but most do not contain parameters for treating metal-ligand complexes. In part this is due to the past focus on pure organic systems, and in part, this is due to the way molecular mechanics models work - a unique set of parameters is required for each different metal ion and each different type of donor atom. When applying the molecular mechanics technique to metal-ligand complexes, one must focus on a class of metal complexes to limit the number of metal ions and donor atom types and then develop a set of parameters before performing the calculations (Hay 1993; Hay and Clement 1998a). 
The MM3 force field (Allinger, Yuh, and Lii 1989) has been extensively parameterized for a wide variety of organic compounds and is one of the most accurate models available. It does not, however, contain parameters required to treat metal complexes. This section describes how the MM3 force field implementation within the commercial software PCModel (Gilbert 2006) was extended to allow the modeling of Kläui ligands and their metal complexes.

Parameters are needed for each unique interaction present in the molecule. Table 4.2 summarizes the possible interaction types, the parameters required to describe them, and the units used in MM3. Further information regarding how these parameters are applied is available elsewhere (Hay 1993).

Table 4.2. Possible Interaction Types, Parameters Required to Describe them, and the Units Used in the MM3 Model

\begin{tabular}{|c|c|c|c|}
\hline Interaction & Parameters & Symbol & Units \\
\hline \multirow[t]{2}{*}{ bond stretch } & preferred length & $r_{0}$ & $\AA$ \\
\hline & force constant & $\mathrm{k}_{\mathrm{r}}$ & mdyne $/ \AA^{2}$ \\
\hline dipole-dipole & bond moment & m & Debye \\
\hline \multirow[t]{2}{*}{ angle bend } & preferred angle & $\theta_{0}$ & deg \\
\hline & force constant & $\mathrm{k}_{\theta}$ & mdyn-A/rad ${ }^{2}$ \\
\hline dihedral angle & barrier heights & $v_{1}, v_{2}, v_{3}$ & $\mathrm{kcal} / \mathrm{mol}$ \\
\hline \multirow[t]{2}{*}{ van der Waals } & radius & $r^{*}$ & $\AA$ \\
\hline & hardness & $\varepsilon$ & $\mathrm{kcal} / \mathrm{mol}$ \\
\hline coulombic & charge & $q$ & esu \\
\hline
\end{tabular}




\subsubsection{The Cyclopentadienyl Anion + Co(III) Complex}

Parameters for the cyclopentadienyl anion ligand and an approach to allow the organometallic species involving this ligand to be calculated within the framework of the MM3 model were published some time ago (Timofeeva et al. 1995). In this parameterization, a new atom type 113 was assigned for the cyclopentadienyl carbon allowing the definition of all bond distances, bond angles, and torsion angles within the ligand. For a description of the metal-ligand interactions ( $\pi$-bonding), a very strong but otherwise ordinary van der Waals potential between the metal and the ligand carbon atoms was used.

Following this approach, a carbon type 113 was defined, and various cyclopentadienyl parameters were added to the default MM3 parameter set of PCModel. This produced a model that reproduced the cyclopentadienyl anion geometry. Next, the Co(III) metal was assigned atom type 66, and the van der Waals distance for interaction of Type 66 with Type 113, $\mathrm{r}^{*}$, was adjusted until the average crystallographic distance of $2.07 \AA$ (Figure 4.6) was reproduced by the model. Figure 4.9 summarizes the parameters for this interaction.

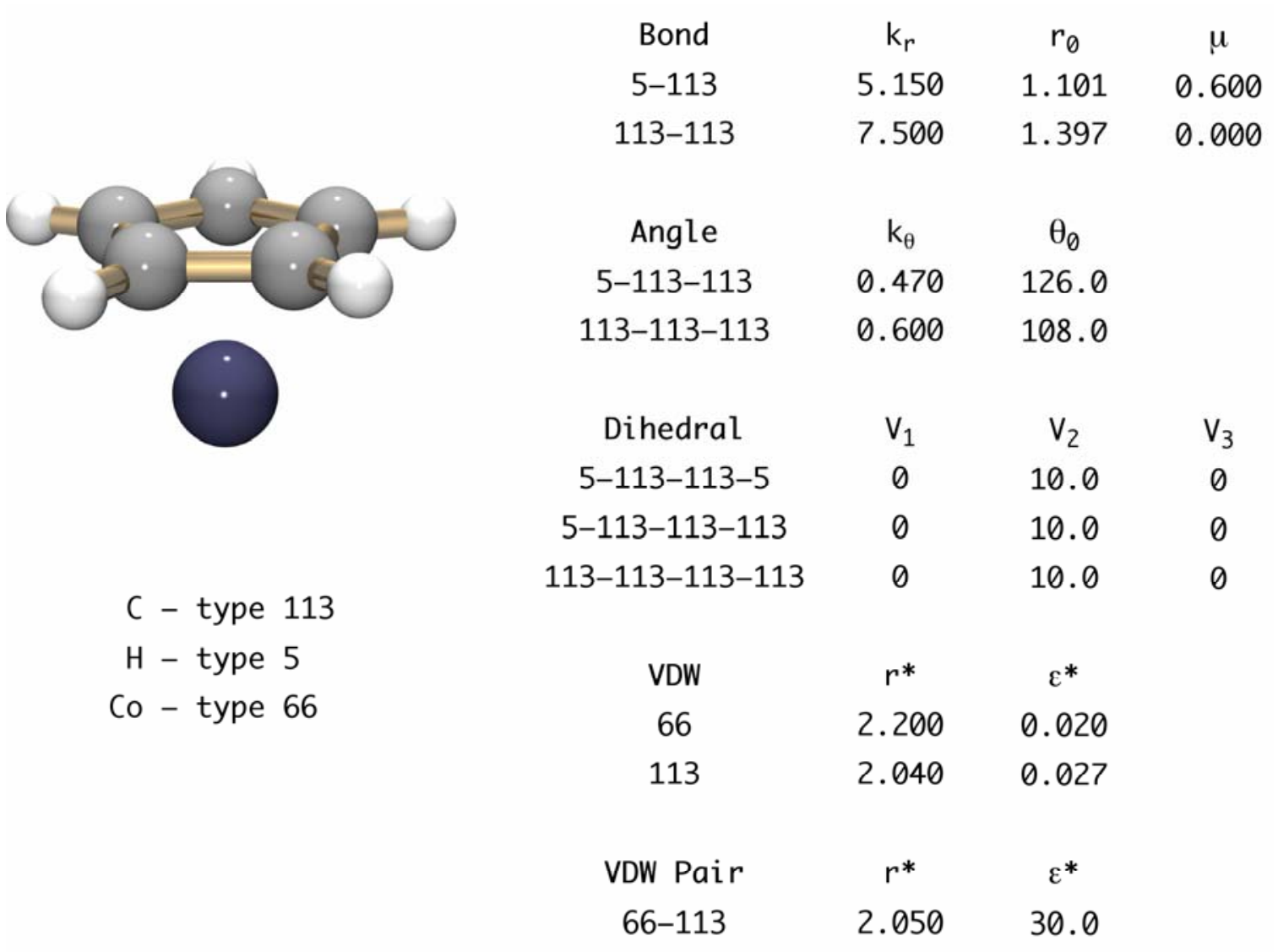

Figure 4.9. The Cyclopentadienyl Anion Ligand and its Complex with Co(III) Can Be Modeled with PCModel After Adding the Above Parameters to the Default MM3 Set 


\subsubsection{Phosphonato Ligand and Co(III) Complex}

The default MM3 force field has been extended to include compounds containing 4-coordinate phosphorus double-bonded to an oxygen and single-bonded to one to three-OR coordinating groups, where $\mathrm{R}=\mathrm{H}$ or $\mathrm{CH}_{3}$ (Stewart et al. 1999). This parameterization, based on electronic structure calculations on phosphinic, phosphonic, and phosphoric acids and related esters, provides a good starting point from which to create a model for the $\mathrm{Co}-\mathrm{P}(=\mathrm{O})(\mathrm{OR})_{2}$ entity. To begin, additional atom types and parameters needed to model $\mathrm{CH}_{3}-\mathrm{P}(=\mathrm{O})(\mathrm{OR})_{2}$ were added to the default $\mathrm{MM} 3$ parameter set and successfully tested. Figure 4.10 summarizes these parameters.

\begin{tabular}{|c|c|c|c|c|}
\hline & Bond & $\mathrm{k}_{\mathrm{r}}$ & $r_{0}$ & $\mu$ \\
\hline & $1-153$ & 3.000 & 1.805 & 0.670 \\
\hline & $7-153$ & 8.900 & 1.510 & -2.500 \\
\hline & $1-159$ & 4.400 & 1.424 & 1.750 \\
\hline & 153-159 & 5.700 & 1.600 & 0.580 \\
\hline & Angle & $k_{\theta}$ & $\theta_{0}$ & \\
\hline & $1-1-153$ & 0.500 & 109.5 & \\
\hline & $5-1-153$ & 0.520 & 109.6 & \\
\hline & $1-1-159$ & 0.830 & 109.5 & \\
\hline & $5-1-159$ & 0.920 & 108.7 & \\
\hline & $1-153-7$ & 0.800 & 117.5 & \\
\hline & $7-153-159$ & 1.180 & 110.5 & \\
\hline & 159-153-159 & 0.900 & 101.0 & \\
\hline C - type 1 & $1-159-153$ & 0.900 & 118.8 & \\
\hline $\mathrm{H}$ - type 5 & & & & \\
\hline$s p^{2} 0-$ type 7 & Dihedral & $v_{1}$ & $v_{2}$ & $v_{3}$ \\
\hline $\mathrm{sp}^{3} 0$ - type 159 & $5-1-153-7$ & 0 & 0 & 0.500 \\
\hline$P$ - type 153 & 5-1-153-159 & 0 & 0 & 0.100 \\
\hline & 5-1-159-153 & 0 & 0 & 0.300 \\
\hline & 1-1-159-153 & 0 & 0 & 0 \\
\hline & $1-153-159-1$ & 1.227 & 0.432 & 1.005 \\
\hline & $7-153-159-1$ & 0 & 0 & 0 \\
\hline & VDW & $r^{*}$ & $\varepsilon^{*}$ & \\
\hline & 153 & 2.200 & 0.168 & \\
\hline & 159 & 1.820 & 0.059 & \\
\hline
\end{tabular}

Figure 4.10. An Alkylphosphonate Molecule Can Be Modeled with PCModel After Adding the Above Parameters to the Default MM3 Set 


\subsubsection{Phosphonato Ligand + Co(III) Complex}

Next, additional stretching, bending, and torsional parameters were added to the parameter set to allow the modeling of the $\mathrm{Co}-\mathrm{P}(=\mathrm{O})(\mathrm{OR})_{2}$ group. A stiff force constant was applied to the Co-P bond, and the distance was set to reproduce the average value observed in crystal structures. Parameters for $\mathrm{Co}-\mathrm{P}-\mathrm{O}$ and $\mathrm{Co}-\mathrm{P}=\mathrm{O}$ angles were set the same as $\mathrm{C}-\mathrm{P}-\mathrm{O}$ and $\mathrm{C}-\mathrm{P}=\mathrm{O}$ angles, and then the preferred angles were adjusted to reproduce the average values observed in crystal structures. Torsional parameters for $\mathrm{Co}-\mathrm{P}-\mathrm{O}-\mathrm{C}$ interactions were set the same as those for $\mathrm{C}-\mathrm{P}-\mathrm{O}-\mathrm{C}$ interactions. This gave a model that well reproduced the average geometric parameters observed for the $\mathrm{Co}-\mathrm{P}(=\mathrm{O})(\mathrm{OR})_{2}$ group in the solid state. Figure 4.11 summarizes the parameters.

\begin{tabular}{|c|c|c|c|c|}
\hline & $\begin{array}{l}\text { Bond } \\
66-153\end{array}$ & $\begin{array}{c}k_{r} \\
10.00\end{array}$ & $\begin{array}{c}r_{0} \\
2.145\end{array}$ & $\begin{array}{c}\mu \\
1.500\end{array}$ \\
\hline & $\begin{array}{c}\text { Angle } \\
153-66-153 \\
7-153-66 \\
66-153-159\end{array}$ & $\begin{array}{c}\mathrm{k}_{\theta} \\
1.500 \\
0.800 \\
0.920\end{array}$ & $\begin{array}{c}\theta_{0} \\
90.0 \\
116.5 \\
111.0\end{array}$ & \\
\hline & Dihedral & $v_{1}$ & $v_{2}$ & $v_{3}$ \\
\hline C - type 1 & $153-66-153-7$ & 0 & 0 & 0.300 \\
\hline H - type 5 & $153-66-153-159$ & 0 & 0 & 0.300 \\
\hline $\begin{array}{l}\mathrm{sp}^{2} 0-\text { type } 7 \\
\mathrm{sp}^{3} 0-\text { type } 159\end{array}$ & $66-153-159-1$ & 1.227 & 0.432 & 1.005 \\
\hline P - type 153 & VDW & $r^{*}$ & $\varepsilon^{*}$ & \\
\hline Co - type 66 & 66 & 2.200 & 0.020 & \\
\hline
\end{tabular}

Figure 4.11. Phosphonate Co(III) Complexes Can Be Modeled with the PCModel After Adding the Above Parameters Once the Default Set Has Been Extended as Shown in Figure 4.10

To attach two or more $\mathrm{P}(=\mathrm{O})(\mathrm{OR})_{2}$ groups to a Co atom, it is necessary to decide how to treat the $\mathrm{P}-\mathrm{Co}-\mathrm{P}$ angles and torsion angles about the $\mathrm{Co}-\mathrm{P}$ bonds. Here we used a normal valence bond treatment for the Co atom; in other words, we defined a specific directionality at the Co center by specifying parameters for the $\mathrm{P}-\mathrm{Co}-\mathrm{P}$ angles: ideal angle of $90^{\circ}$ and a force constant of $1.50 \mathrm{mdyn}-\AA / \mathrm{rad}^{2}$. The torsion parameters for rotation about $\mathrm{Co}-\mathrm{P}$ bonds were set to give a slight preference for a staggered conformation, $\mathrm{V}_{3}=0.300 \mathrm{kcal} / \mathrm{mol}$. Figure 4.11 also summarizes the parameters. 


\subsubsection{Testing the Model's Ability to Treat the Kläui Ligand}

With the parameterization thus far, it is possible to calculate the structure of the complete Kläui ligand. This was done as an intermediate check to see how well our model was reproducing the average crystal structure geometry. Figure 4.12 provides a picture of the MM3 optimized structure and compares the calculated and observed geometric parameters. It can be seen that average experimental bond lengths and angles are well reproduced.

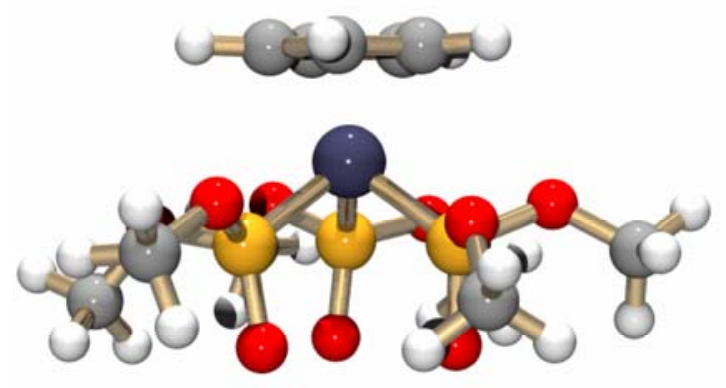

$\begin{array}{ccc}\text { Distances } & \text { Calc }(A) & \text { Obs }(\AA) \\ \text { Co-C } & 2.08 & 2.07 \pm 0.02 \\ \text { C-C } & 1.40 & 1.39 \pm 0.04 \\ \text { P-Co } & 2.16 & 2.16 \pm 0.02 \\ \text { P-0 } & 1.61 & 1.60 \pm 0.04 \\ \text { P=0 } & 1.51 & 1.51 \pm 0.03 \\ \text { C-0 } & 1.43 & 1.41 \pm 0.07\end{array}$

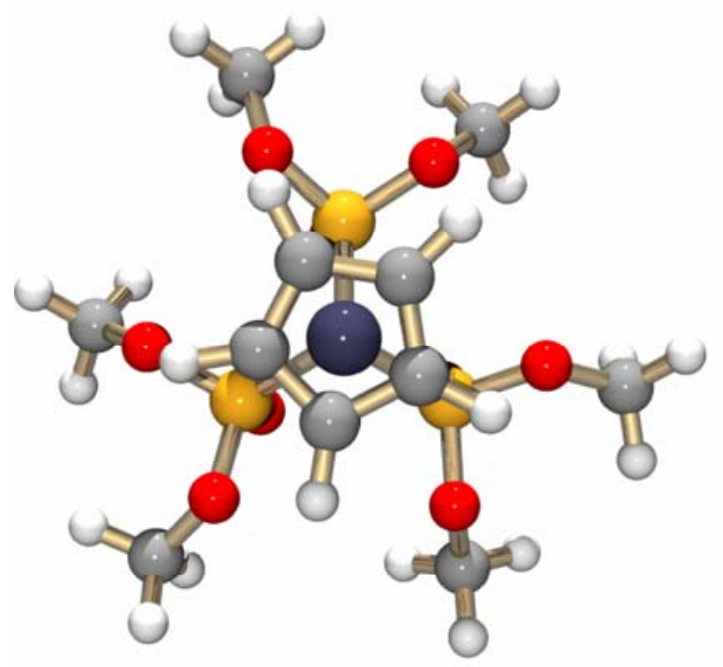

$\begin{array}{ccc}\text { Angles } & \text { Calc }\left({ }^{\circ}\right) & \text { Obs }\left({ }^{\circ}\right) \\ \text { C-C-C } & 108 & 108 \pm 3 \\ \text { P-Co-P } & 92 & 91 \pm 2 \\ \text { Co-P-0 } & 109 & 109 \pm 4 \\ \text { Co-P=0 } & 119 & 119 \pm 2 \\ \text { 0-P-0 } & 101 & 101 \pm 4 \\ \text { 0-P=0 } & 109 & 108 \pm 2 \\ \text { P-0-C } & 122 & 123 \pm 4\end{array}$

Figure 4.12. Views of the MM3 Optimized Kläui Ligand (left) and a Comparison of Calculated Versus Observed Structural Features (right) 


\subsubsection{Kläui-Metal Ion Complexes}

The final step in the parameterization involved adding the interactions between the Kläui ligand and a metal ion, M. A points-on-a-sphere approach was used to model the coordination sphere about M (Hay 1993). In other words, bond angles subtended at $M$ are not specifically defined. Instead, these angle terms are replaced by van der Waals interactions between all atoms directly attached to $\mathrm{M}$. With this approach, the only remaining interactions missing parameters are $\mathrm{M}-\mathrm{O}$ stretch, $\mathrm{M}-\mathrm{O}=\mathrm{P}$ bend, $\mathrm{M}-\mathrm{O}=\mathrm{P}-\mathrm{O}$ torsion, and $\mathrm{M}-\mathrm{O}=\mathrm{P}-\mathrm{Co}$ torsion.

Kläui crystal structures that can be used to validate the performance of the model contain a variety of metal ion guests. In addition, structural data for Kläui-metal ion complexes of interest are not available in some cases. Thus, it was desirable to define the required metal-dependent parameters as a function of metal ion property such as the formal charge, Z, the ionic radius (IR) (Shannon 1976), and Pauling's electronegativity (EN) (http://www.webelements.com). Relationships developed in a prior study of metal-amide complexes (Hay et al. 1998b) were adapted for application to Kläui complexes.

The resulting equations for $\mathrm{M}-\mathrm{O}$ stretch parameters and $\mathrm{M}-\mathrm{O}=\mathrm{P}$ bending parameters, shown in Figure 4.13, were obtained as follows. It was assumed that the stretching force constant, $\mathrm{k}_{\mathrm{r}}$, was given by Equation 1. The preferred bond lengths, $\mathrm{r}_{0}$, were assumed to be equal to the ionic radius plus a constant as shown in Equation 2. The value for this constant was determined empirically after a series of calculations on a test set of Kläui complexes taken from crystal structures (see next section). Stretching parameters obtained from Equations 1 and 2 reproduced the experimental $\mathrm{M}-\mathrm{O}$ distances in the test set of Kläui complexes, which ranged from 1.87 to $2.47 \AA$, to an average deviation of $\pm 0.02 \AA$.

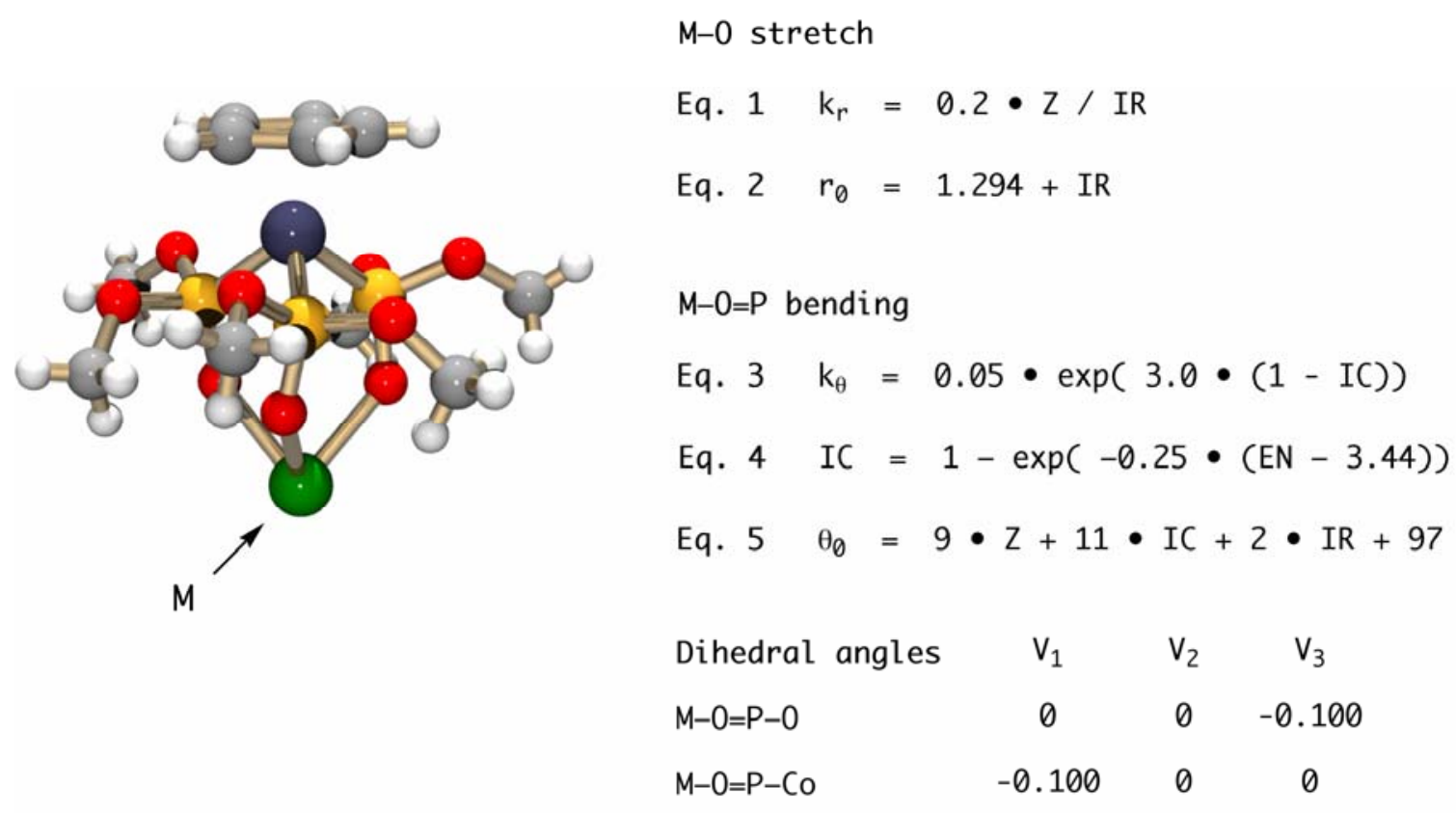

Figure 4.13. Metal-Dependent Parameters for Modeling Kläui-Metal Complexes. Stretching and bending parameters are obtained from empirical relationships using metal ion properties such as the charge, $\mathrm{Z}$, the ionic radius, IR, and the electronegativity, EN. 
It was assumed that the bending force constant, $\mathrm{k}_{\theta}$, is related to the ionic character (IC) of the metal ion, as in Equation 3, where IC is calculated from the electronegativity of the element using Equation 4 (Pauling 1960). Based on the behavior of other terminal oxygen donor atoms, the ideal angle, $\theta_{0}$, will depend on the degree of covalency, with more covalent metals giving more acute angles. The degree of covalency decreases as the charge, size, and ionic character of the metal ion increases. This is reflected in Equation 5, where again the coefficients were obtained empirically after a series of calculations on a test set of Kläui complexes taken from crystal structures (see next section). Bending parameters obtained from Equations 3 and 4, reproduced experimental $\mathrm{M}-\mathrm{O}=\mathrm{P}$ angles in Kläui complexes, which ranged from 121 to $138^{\circ}$, to an average deviation of $\pm 1.1^{\circ}$.

Dihedral angle parameters involving $\mathrm{M}$ were assumed to be constant for all metal ions. They were set to give a small preference for the $\mathrm{M}-\mathrm{O}$ bond to eclipse the $\mathrm{P}-\mathrm{Co}$ bond as observed in crystal structure data (see Figure 4.8 above). Figure 4.13 also gives these parameters.

\subsubsection{Validation of the Extended MM3 Model}

Sixteen Kläui-metal complexes were selected from the available crystal structures to use for developing and validating the MM3 model. The structures consist of:

- 2:1 Kläui-metal complexes where there are no other ligands attached to the metal [six cases representing metal ions $\mathrm{Mn}(\mathrm{II}), \mathrm{Mn}(\mathrm{III}), \mathrm{Ni}(\mathrm{II}), \mathrm{Ga}(\mathrm{III}), \mathrm{Zr}(\mathrm{IV})$, and $\mathrm{Hg}(\mathrm{II})$ ]

- 2:1 Kläui-metal complexes where there are additional unidentate ligands in the coordination sphere [four examples representing metal ions La(III), Eu(III), and U(IV)]

- 1:1 Kläui-metal complexes in which the coordination sphere is completed by unidentate ligands [six examples representing metal ions Ti(IV), Ge(IV), Zr(IV), and $\mathrm{Pt}(\mathrm{II})$ ].

Table 4.3 summarizes properties for the metal ions contained in these complexes, specific $\mathrm{M}-\mathrm{O}$ stretching, and $\mathrm{M}-\mathrm{O}=\mathrm{P}$ bending parameters computed using the relationships given in Figure 4.13.

In cases where unidentate ligands were also present in the coordination sphere, we used a simple, but effective, method to model them (Hay and Rustad 1994; Hay et al. 1998b; Hay et al. 1998c; Hay et al. 2004). That is, the unidentate ligand, $\mathrm{L}$, was reduced to a single atom attached to the metal, $\mathrm{M}$, and parameters for the $\mathrm{M}-\mathrm{L}$ stretch were defined as follows: $\mathrm{r}_{0}=$ average observed $\mathrm{M}-\mathrm{L}$ distance and $\mathrm{k}_{\mathrm{r}}=10$ $\mathrm{mdyn} / \AA$. This approach minimizes the need for additional parameterization and gives a model that accounts for the steric influence of the unidentate ligands on the inner coordination sphere. 
Table 4.3. Physical Properties and Metal-Dependent Parameters for Selected Metal Ions

\begin{tabular}{|c|c|c|c|c|c|c|c|c|}
\hline Metal CN & IR & $\mathbf{Z}$ & EN & IC & $\mathbf{r}_{\mathbf{0}}$ & $\mathbf{k}_{\mathbf{r}}$ & $\theta_{0}$ & $\mathbf{k}_{\theta}$ \\
\hline $\mathrm{Ge}(\mathrm{IV}) 6$ & 0.530 & 4 & 2.01 & 0.400 & 1.824 & 1.509 & 138.4 & 0.302 \\
\hline $\operatorname{Ti(IV)~} 6$ & 0.605 & 4 & 1.54 & 0.594 & 1.899 & 1.322 & 140.7 & 0.168 \\
\hline $\mathrm{Ga}(\mathrm{III}) 6$ & 0.620 & 3 & 1.81 & 0.485 & 1.914 & 0.967 & 130.5 & 0.234 \\
\hline $\operatorname{Mn}(\mathrm{III}) 6$ & 0.645 & 3 & 1.55 & 0.590 & 1.939 & 0.930 & 131.7 & 0.170 \\
\hline $\mathrm{Fe}(\mathrm{III}) 6$ & 0.645 & 3 & 1.83 & 0.476 & 1.939 & 0.930 & 130.5 & 0.240 \\
\hline $\mathrm{Ni}(\mathrm{II}) \quad 6$ & 0.690 & 2 & 1.91 & 0.443 & 1.984 & 0.579 & 121.2 & 0.265 \\
\hline $\operatorname{Zr}(\mathrm{IV}) 6$ & 0.720 & 4 & 1.33 & 0.671 & 2.014 & 1.111 & 141.8 & 0.133 \\
\hline $\operatorname{Pt}(\mathrm{II}) \quad 6$ & 0.800 & 2 & 2.28 & 0.285 & 2.094 & 0.500 & 119.7 & 0.426 \\
\hline $\operatorname{Mn}(\mathrm{II}) 6$ & 0.830 & 2 & 1.55 & 0.590 & 2.124 & 0.481 & 123.1 & 0.170 \\
\hline $\mathrm{Pu}(\mathrm{IV}) 8$ & 0.960 & 4 & 1.28 & 0.688 & 2.254 & 0.833 & 142.4 & 0.127 \\
\hline $\mathrm{U}(\mathrm{IV}) \quad 8$ & 1.000 & 4 & 1.38 & 0.653 & 2.294 & 0.800 & 142.1 & 0.141 \\
\hline $\operatorname{Hg}(\mathrm{II}) \quad 6$ & 1.020 & 2 & 2.00 & 0.404 & 2.314 & 0.392 & 121.4 & 0.298 \\
\hline Th(IV) 8 & 1.050 & 4 & 1.30 & 0.681 & 2.344 & 0.761 & 142.6 & 0.129 \\
\hline $\mathrm{Eu}(\mathrm{III}) 8$ & 1.066 & 3 & 1.18 & 0.721 & 2.360 & 0.562 & 134.0 & 0.115 \\
\hline $\mathrm{La}(\mathrm{III}) 8$ & 1.160 & 3 & 1.10 & 0.745 & 2.454 & 0.517 & 134.5 & 0.107 \\
\hline
\end{tabular}

$\mathrm{CN}=$ number of atoms directly attached to the metal

$\mathrm{IR}=$ ionic radius (Shannon 1976)

$\mathrm{Z}=$ formal charge

$\mathrm{EN}=$ Pauling's electronegativity [http://webelements.com]

$\mathrm{IC}=$ ionic character calculated using Equation 5 in Figure 4.14.

As noted in the preceding section, the metal-dependent parameters given in Table 4.3 reproduce $\mathrm{M}-\mathrm{O}$ distances with an average deviation of $\pm 0.02 \AA$ and $\mathrm{M}-\mathrm{O}=\mathrm{P}$ angles with an average deviation of $\pm 1.1^{\circ}$ for the 16 Kläui-metal complexes. Moreover, it was found that the model reproduces the average bond distances and angles within the Kläui ligand itself (see Figure 4.12). A final test of the model accuracy was obtained by superposition of observed and calculated structures.

Starting from their crystal structure coordinates, the 16 complexes in the test set were calculated with the extended MM3 model described above. The root-mean-squared deviation, rmsd, between the calculated and experimental atom positions for all non-hydrogen atoms provides a yardstick for assessing how well the structure is reproduced. Figure 4.14 presents overlays of experimental and calculated structures.

The rmsd values ranged from 0.153 to $0.740 \AA$, with a mean value of $0.391 \AA$ for the entire set. The core of each complex, i.e., the inner coordination sphere around the ligated metal ion, is well reproduced. When present, significant discrepancies between calculated and observed structures result primarily from the orientation of ethyl side arms. Structural variations in the ethyl side arms can be attributed to 1) the presence of multiple low lying conformational states (evidenced by disorder reported for 7 out of the 16 crystal structures - specific cases are denoted in Figure 4.14) and 2) distortions resulting from crystal packing and lattice interactions. 

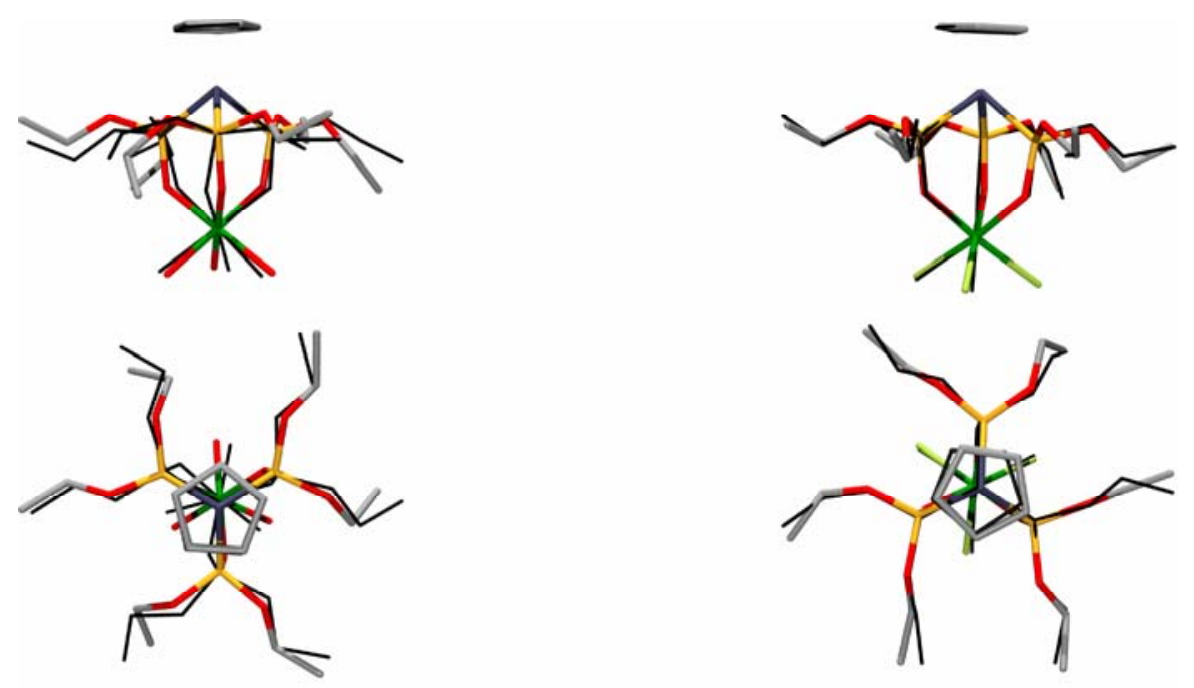

Ti(IV), rmsd 0.503 Å

Ti(IV), rmsd $0.288 \AA$

FESPID, $r_{\text {fac }}=0.048$

XACBUZ, $\mathrm{r}_{\mathrm{fac}}=0.057$

Disorder in ethyl groups.
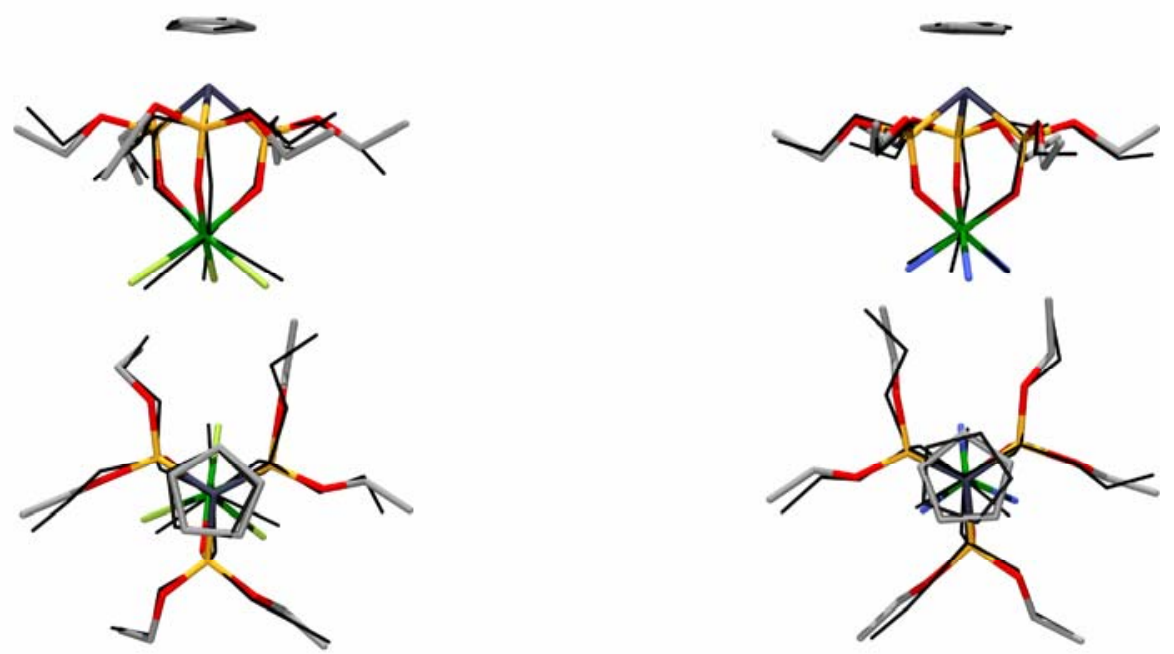

Zr(IV), rmsd 0.433 A

Ge(IV), rmsd $0.410 \AA$

SURVOQ, $\mathrm{r}_{\mathrm{fac}}=0.029$

LOBCAG, $r_{\text {fac }}=0.052$

Disorder in ethyl groups.

Figure 4.14. Superpositions of X-ray Crystal Structure Geometries (colored) and Calculated Geometries (black) of Kläui-Metal Complexes. Two views are given for each complex. Hydrogen atoms not shown. 

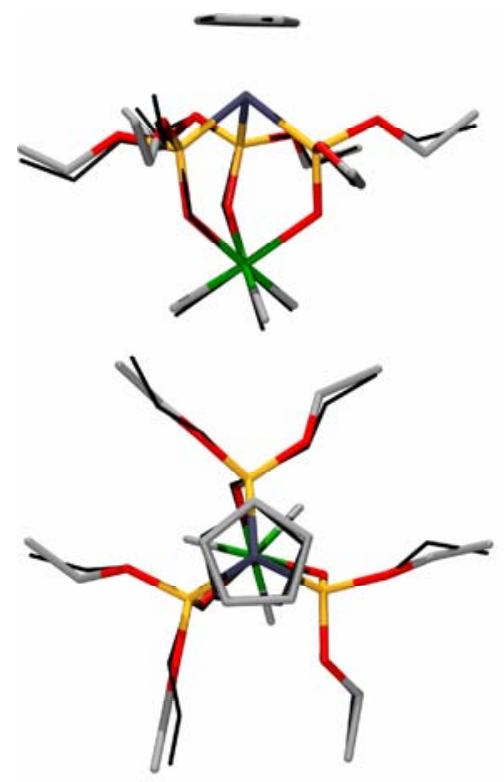

Pt(II), rmsd 0.250 Å

JIBVIZ, $\mathrm{r}_{\text {fac }}=0.032$
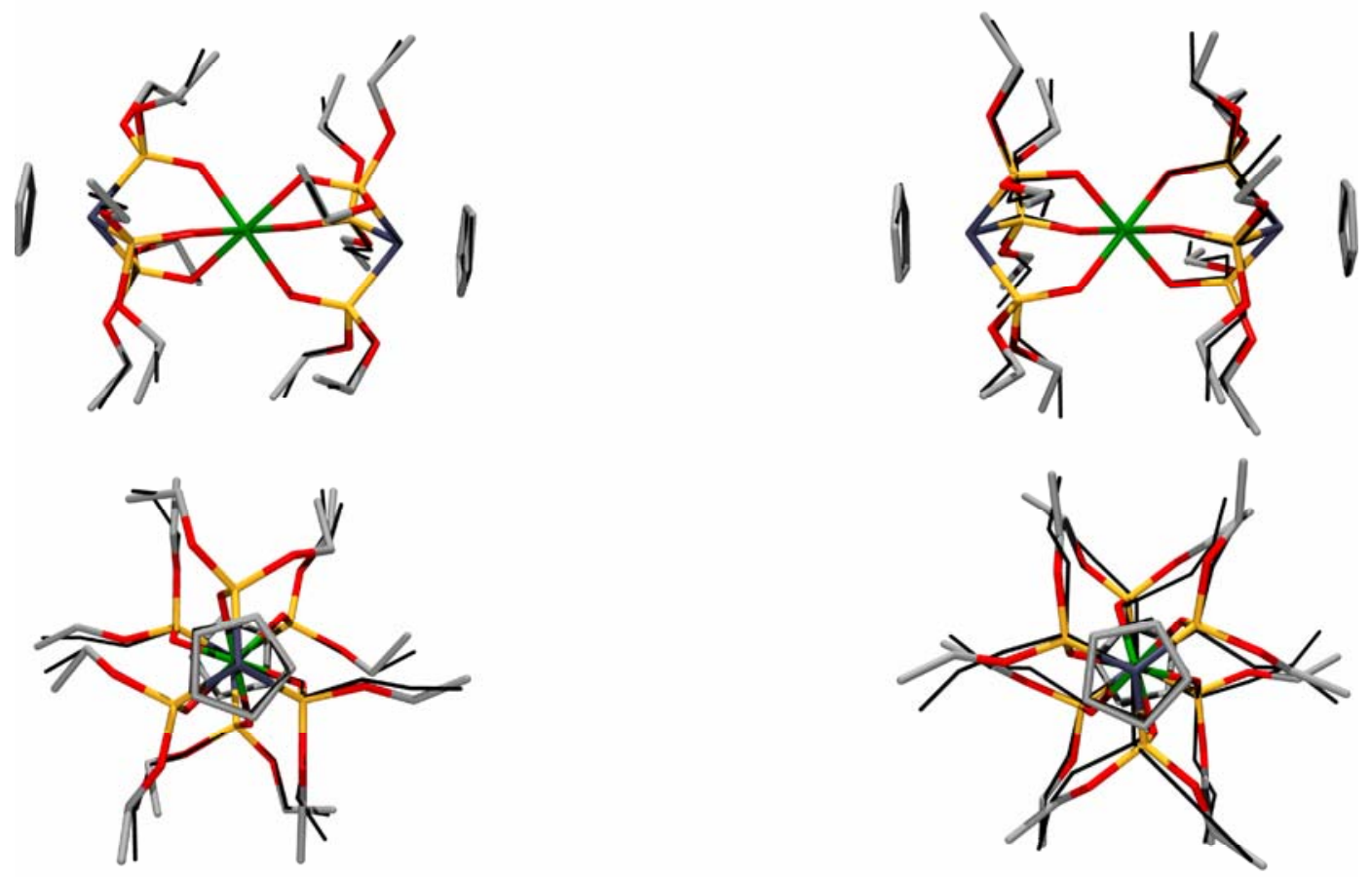

Mn(II), rmsd 0.266 A

MESVUB, $\mathrm{r}_{\mathrm{fac}}=0.077$

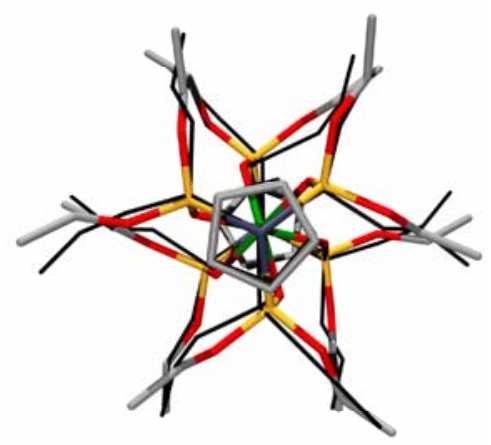

Mn(III), rmsd $0.330 \AA$

MESWAI, $\mathrm{r}_{\mathrm{fac}}=0.077$

Disorder in ethyl groups.

Figure 4.14 (Contd) 

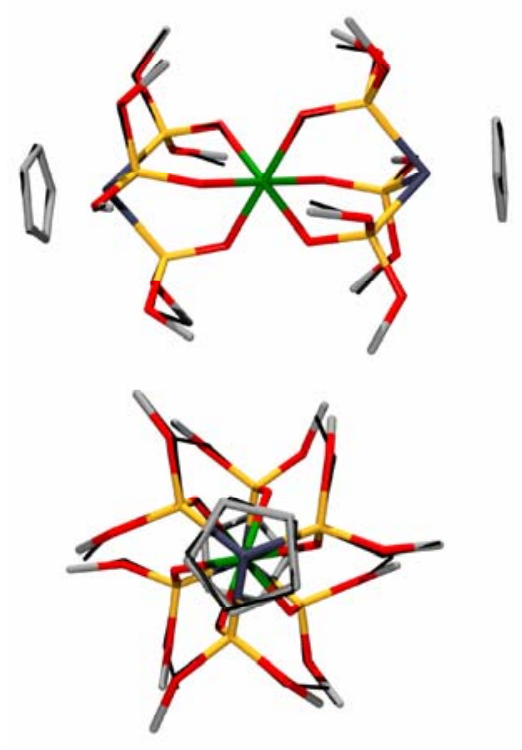

$\mathrm{Ni}(\mathrm{II})$, rmsd $0.153 \AA$

MECPUF, $\mathrm{r}_{\mathrm{fac}}=0.073$
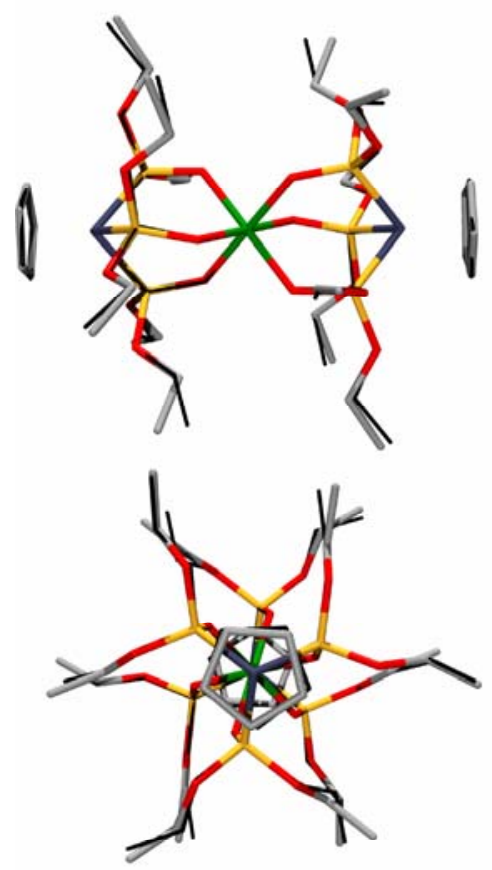

Ga(III), rmsd $0.267 \AA$

YEZCIP, $r_{\text {fac }}=0.060$

Disorder in cp ring.
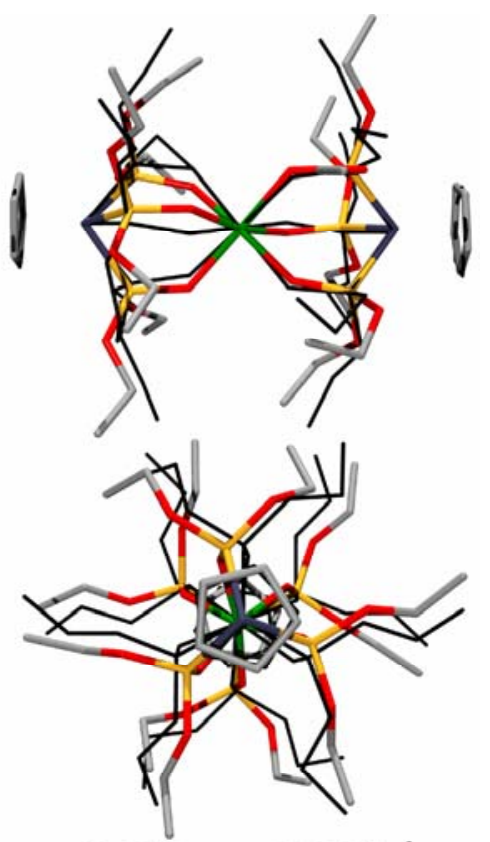

Zr(IV), rmsd 0.740 A

SURSAZ, $r_{\text {fac }}=0.045$
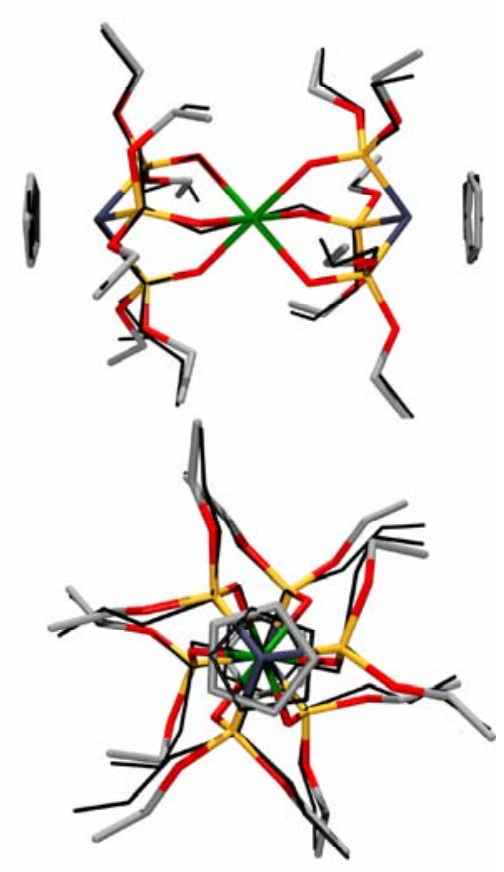

Hg(II), rmsd $0.386 \AA$

SARSAF, $\mathrm{r}_{\mathrm{fac}}=0.056$

Disorder in ethyl groups.

Figure 4.14 (Contd) 

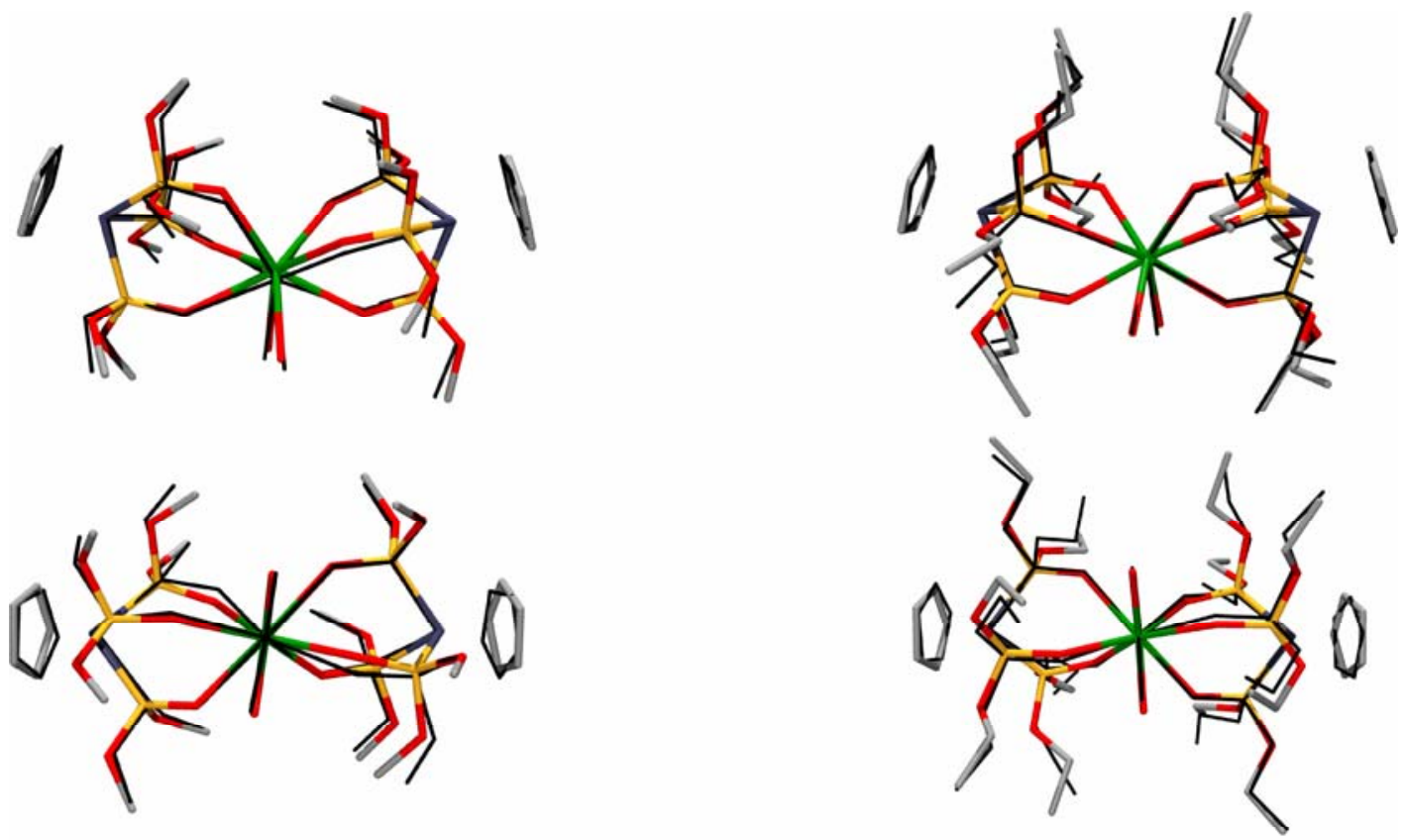

La(III), rmsd 0.313 Å

Eu(III), rmsd 0.563Å

ZENGII, $r_{\text {fac }}=0.039$

GENPEU, $r_{\text {fac }}=0.073$
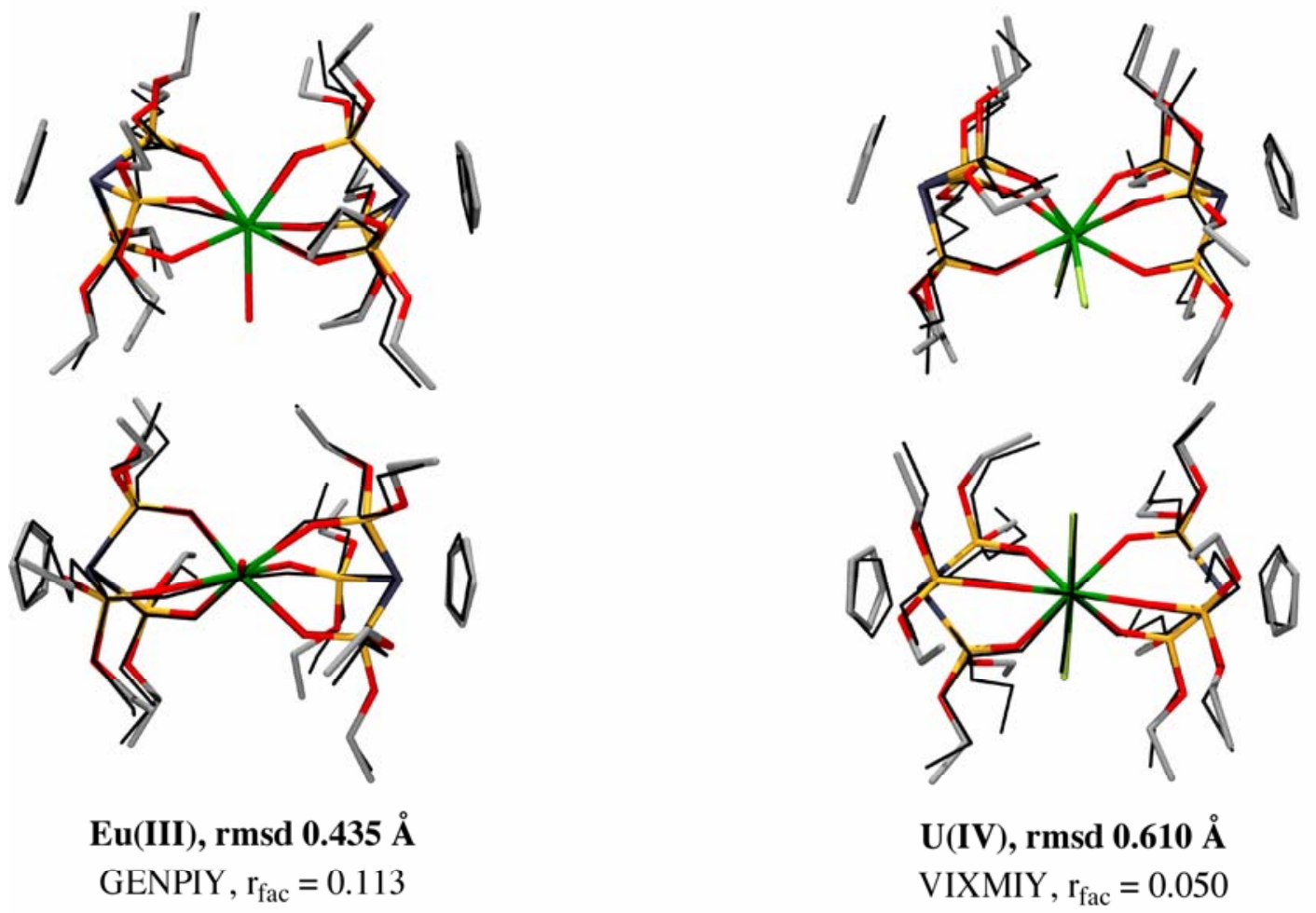

Figure 4.14 (Contd) 


\subsubsection{Predicted Geometry for a $\left[\mathrm{Pu}(\mathrm{Kläui})_{2}\left(\mathrm{OH}_{2}\right)\right]^{2+} \mathrm{Complex}$}

Given the extended MM3 model described above in Section 4.2.2, it is possible to predict geometries for complexes where no experimental data exist. Of particular interest is the geometry for an eightcoordinate $\mathrm{Pu}(\mathrm{IV})$ complex. This structural data are required as input for the next step in the design process, de novo structure-based design via the HostDesigner program. The MM3 optimized geometry, Figure 4.15 left, shows the expected tilted orientation of the two Kläui ligands (see Section 4.1.2). The Kläui ligand oxygen atoms sit on the surface of a sphere with an average radius of $2.31 \AA$.

Also of interest is the geometry of the complex formed by the competing Fe(III) ion. These structural data are useful in the scoring stage of the design process - candidate architectures must complement $\mathrm{Pu}(\mathrm{IV})$, but not $\mathrm{Fe}(\mathrm{III})$. The MM3 optimized geometry, Figure 4.15 right, shows the expected parallel orientation of the two Kläui ligands (see Section 4.1.2). Here the Kläui ligand oxygen atoms sit on the surface of a sphere with a radius of $2.00 \AA$.
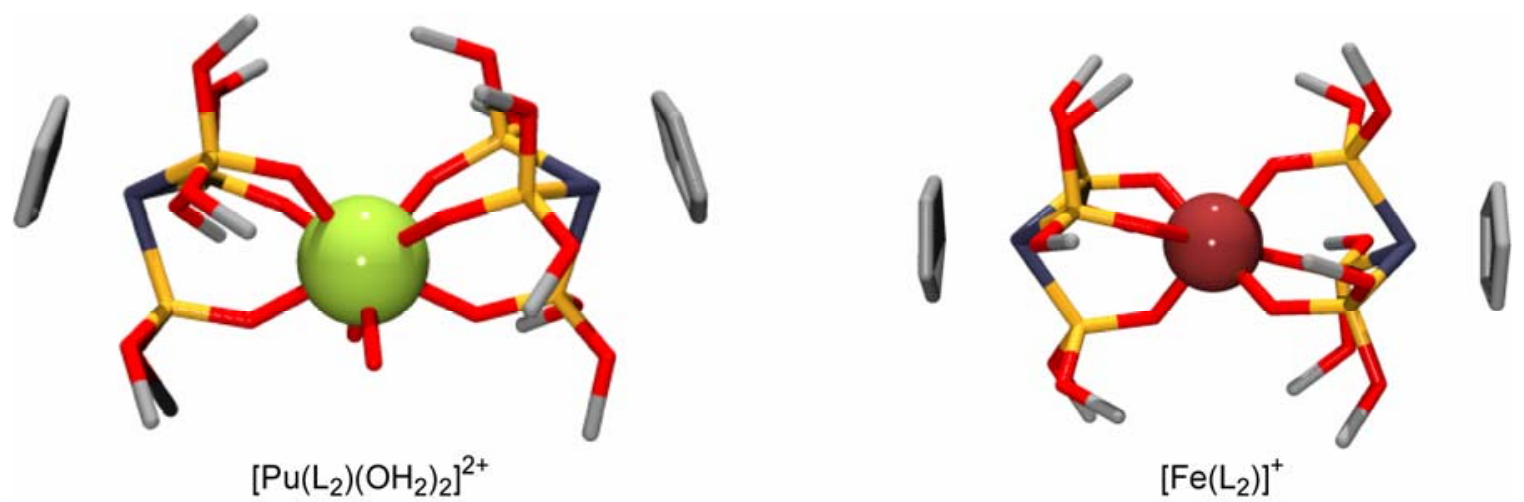

Figure 4.15. $\mathrm{MM} 3$ Optimized Geometries for $\left[\mathrm{Pu}(\mathrm{Kläui})_{2}\left(\mathrm{OH}_{2}\right)_{2}\right]^{2+}$ (left) and $\left[\mathrm{Fe}(\mathrm{Kläui})_{2}\right]^{+}$(right). Hydrogen atoms not shown.

\subsection{Summary}

This initial study has completed the groundwork necessary to perform the computer-aided design of bis-Kläui architectures tailored for $\mathrm{Pu}(\mathrm{IV})$ complexation. Structural features of the Kläui ligand and its metal complexes have been surveyed and analyzed. An extended molecular mechanics model, MM3, has been developed and validated for application to these structures. The MM3 model allows both 1) the prediction of geometries required as input to the design process and 2) a rapid means of evaluating candidate architectures.

The next step in the design process is to use de novo molecule building software (HostDesigner) to identify potential candidate architectures. Initial scoring would be based solely on geometry. Subsequent scoring would involve the use of the MM3 model to rank structures based on their degree of fit to $\mathrm{Pu}(\mathrm{IV})$ versus degree of fit to Fe(III). The result would be a list of molecular structures that would be expected to show enhanced binding and selectivity for $\mathrm{Pu}(\mathrm{IV})$. It would then be necessary to synthesize and characterize these ligands. 


\subsection{Conclusions and Recommendations}

In this work, we have investigated new materials for potential use in automated radiochemical separations. The primary conclusions and recommendations from this work are as follows.

- The synthesis of Kläui ligand derivatives containing functionalized pendant groups on the cyclopentadienyl ring is feasible. These functional groups should allow covalent binding of the Kläui ligand to polymeric resins or other surfaces. A modified synthetic scheme to achieve this has been proposed and will be pursued in FY 2007.

- Work was initiated on the synthesis of bicyclic diamides functionalized for binding to polymeric resins or other surfaces. This work is being performed in collaboration with researchers at the University of Oregon. To date, this effort has focused on synthesizing and characterizing a symmetrically substituted bicyclic diamide ligand with the $-\mathrm{COOH}$ functionality.

- Extraction chromatography resins prepared by sorption of Kläui ligand onto an inert macroreticular polymeric support display high affinity for tetravalent actinides. These materials also sorb trivalent actinides at low nitric acid concentrations, but the affinity for the trivalent actinides decreases with increasing $\left[\mathrm{HNO}_{3}\right]$. These materials have relatively low affinity for $\mathrm{U}(\mathrm{VI})$, but they do sorb U(VI) to a greater extent than $\mathrm{Am}(\mathrm{III})$ at $\left[\mathrm{HNO}_{3}\right]>0.3 \mathrm{M}$. Preliminary results suggest that the Kläui resins can separate $\mathrm{Pu}(\mathrm{IV})$ from sample solutions containing high concentrations of competing ions. Conceptual protocols to recover the $\mathrm{Pu}$ from the resin for subsequent analysis have been proposed, but further work is needed to perfect these techniques. Work on this subject will be continued in FY 2007. Automated laboratory equipment (in conjunction with Task 3 of the NA-22 Automation Project) will be used in FY 2007 to improve the efficiency of these experiments.

- An extended MM3 molecular mechanics model has been developed for calculating the structures of Kläui ligand complexes. This has laid the groundwork necessary to perform the computeraided design of bis-Kläui architectures tailored for $\mathrm{Pu}(\mathrm{IV})$ complexation. The next step in the design process is to use de novo molecule building software (HostDesigner) to identify potential candidate architectures. Initial scoring would be based solely on geometry. Subsequent scoring would involve the use of the MM3 model to rank structures based on their degree of fit to $\mathrm{Pu}(\mathrm{IV})$ versus degree of fit to Fe(III). The result would be a list of molecular structures that would be expected to show enhanced binding and selectivity for $\mathrm{Pu}(\mathrm{IV})$. It would then be necessary to synthesize and test these ligands. 


\subsection{References}

Allen FH, and O Kennard. 1993. Chemical Design Automation News 8:31. Cambridge Structural Database, Version 5.27, November 2005, Cambridge Crystallographic Data Centre, 12 Union Road, Cambridge, CB2 1EZ, UK. Available at: http://www.ccdc.cam.ac.uk/products/csd/. Accessed 10-17-06.

Allinger NL, YH Yuh, J-H Lii. 1989. "Molecular Mechanics: The MM3 Force Field for Hydrocarbons." J. Am. Chem. Soc. 111:8551-8566.

Birnbaum JC, B Busche, Y Lin, WJ Shaw, and GE Fryxell. 2002. "Synthesis of Carbamoylphosphonate Silanes for the Selective Sequestration of Actinides." Chem. Comm. 2002:1374-1375.

Bryantsev VS, and BP Hay. 2006. "De Novo Structure-Based Design of Bis-Urea Architectures for Anion Recognition." J. Am. Chem. Soc. 128:2035-2042.

Burkert U, and NL Allinger. 1982. Molecular Mechanics. American Chemical Society, Washington, DC.

Fryxell GE, Y Lin, S Fiskum, JC Birnbaum, H Wu, K Kemner, and S Kelly. 2005. “Actinide Sequestration Using Self-Assembled Monolayers on Mesoporous Supports." Environ. Sci. Technol. 39:1324-1331.

Gilbert KE. 2006. PCModel, Version 9, Serena Software, Bloomington, IN 97402. Available at: http://www.serenasoft.com/. Accessed 10-17-06.

Hart WP, DW Macomber, and MD Rausch. 1980. “A New, General Route to Functionally Substituted $\eta^{5}$-Cyclopentadienyl Metal Compounds.” J. Am. Chem. Soc. 102:1196-1198.

Hay BP. 1993. "The Application of Molecular Mechanics in Coordination Chemistry." Coordination Chemistry Reviews 126:177-236.

Hay BP, and JR Rustad. 1994. "Structural Criteria for the Rational Design of Selective Ligands: Extension of the MM3 Force Field to Aliphatic Ether Complexes of the Alkali and Alkaline Earth Cations." J. Am. Chem. Soc. 116:6316-6326.

Hay BP, and O Clement. 1998a. "Metal Complexes." In: The Encyclopedia of Computational Chemistry, PvR Schleyer, NL Allinger, T Clark, J Gasteiger, PA Kollman, HF Schaefer III, and PR Schreiner (Eds.); John Wiley \& Sons, Chichester, UK.

Hay BP, O Clement, G Sandrone, and DA Dixon. 1998b. “A Molecular Mechanics (MM3(96)) Force Field for Metal-Amide Complexes.” Inorg. Chem. 37:5887-5894.

Hay BP, L Yang, J-H Lii, and NL Allinger. 1998c. “An Extended MM3(96) Force Field for Complexes of the Group 1A and 2A Cations with Ligands Bearing Conjugated Ether Donor Groups.” Journal of Molecular Structure, THEOCHEM 428:203-219. 
Hay BP, and TK Firman. 2002. "HostDesigner: A Program for the de Novo Structure-Based Design of Molecular Receptors with Binding Sites that Complement Metal Ion Guests." Inorg. Chem.

41:5502-5512. Available at: http://hostdesigner.emsl.pnl.gov. Accessed 10-17-06.

Hay BP, TK Firman, GJ Lumetta, BM Rapko, PA Garza, SI Sinkov, JE Hutchison, BW Parks, RD Gilbertson, and TJR Weakley. 2004. "Toward the Computer-Aided Design of Metal Ion Sequestering Agents. Journal of Alloys and Compounds 374:416-419.

Hay BP, J Uddin, and TK Firman. 2004. "Eight-coordinate Stereochemistries of U(IV) Catecholate and Aquo Complexes." Polyhedron 23:145-154.

Hay BP, AA Oliferenko, J Uddin, C Zhang, and TK Firman. 2005. "Search for Improved Host Architectures: Application of De Novo Structure-Based Design and High Throughput Screening Methods to Identify Optimal Building Blocks for Multidentate Ethers.” J. Am. Chem. Soc. 127:17043-17053.

Kläui W, H Neukomm, H Werner, and G Huttner. 1977. " $\left[\pi-\mathrm{C}_{5} \mathrm{H}_{5} \mathrm{Co}\left\{\mathrm{P}(\mathrm{OR})_{2} \mathrm{O}\right\}_{3} \mathrm{BF}\right] \mathrm{BF} \mathrm{F}_{4}$ : Synthese, Struktur und Reaktivität von (Cyclopentadienyl)kobalt-Komplexen mit neuartigen käfigbildenden Phosphit-Chelatliganden." Chem. Ber. 110:2283-2289.

Lin Y, SK Fiskum, W Yantasee, H Wu, SV Mattigod, E Vorpagel, GE Fryxell, KN Raymond, and J Xu. 2005. "Incorporation of Hydroxypyridinone Ligands into Self-Assembled Monolayers on Mesoporous Supports for Selective Actinide Sequestration.” Environ. Sci. Technol. 39:1332-1337.

Lin Y, GE Fryxell, H Wu, and M Engelhard. 2001. "Selective Sorption of Cesium Using SelfAssembled Monolayers on Mesoporous Supports.” Environ. Sci. Technol. 35:3962-3966.

Lumetta GJ, BK McNamara, TL Hubler, DW Wester, J Li, and SL Latesky. 2006. "Potential Application of Kläui Ligands in Actinide Separations." In: Separations for the Nuclear Fuel Cycle in the $21^{\text {st }}$ Century, ACS Symposium Series, American Chemical Society, Washington, DC; pp. 201-218.

Lumetta GJ, DW Wester, BK McNamara, TL Hubler, SL Latesky, CC Martyr, and KN Richards. 2004. "A New Extraction Chromatography Resin Containing Kläui Ligands for Application in Actinide Separations." Solvent Extr. Ion Exch. 22:947-960.

Lumetta GJ, BM Rapko, PA Garza, BP Hay, RD Gilbertson, TJR Weakley, and JE Hutchison. 2002. "Deliberate Design of Ligand Architecture Yield Dramatic Enhancement of Metal Ion Affinity." J. Am. Chem. Soc. 124:5644-5645.

Lumetta GJ, BM Rapko, BP Hay, PA Garza, JE Hutchison, and RD Gilbertson. 2003. "A Novel Bicyclic Diamide With High Binding Affinity For Trivalent f-Block Elements." Solvent Extr. Ion Exch. 21:29-39.

Pauling L. 1960. The Nature of the Chemical Bond. Cornell University Press, New York. 
Román EE, CF Tapia, and MS Hernández. 1986. "A Novel Access to the Anionic Permethylated facTripod Ligand $\left[\mathrm{C}_{5} \mathrm{Me}_{5} \mathrm{Co}\left\{\mathrm{P}(\mathrm{O})(\mathrm{OEt})_{2}\right\}_{3}\right]^{\prime}$, and its Derived Trinuclear and Binuclear Complexes." Polyhedron 5:917-920.

Ryan JL. 1960. "Species Involved in the Anion-Exchange Absorption of Quadrivalent Actinide Nitrates." J. Phys. Chem. 64:1375-1385.

Schneider H-J, and A Yatsimirsky. 2000. Principles and Methods in Supramolecular Chemistry, John Wiley and Sons, New York.

Shannon RD. 1976. "Revised Effective Ionic Radii and Systematic Studies of Interatomic Distances in Halides and Chalcogenides." Acta Crystallographica A32:751-767.

Steed JW, and JL Atwood. 2000. Supramolecular Chemistry. John Wiley and Sons, New York.

Stewart EL, N Nevins, NL Allinger, and JP Bowen. 1999. "Molecular Mechanics (MM3) Calculations on Oxygen-Containing Phosphorus (Coordination IV) Compounds." Journal of Organic Chemistry 64:5350-5360

Timofeeva TV, J-H Lii, and NL Allinger. 1995. "Molecular Mechanics Explanation of the Metallocene Bent Sandwich Structure.” J. Am. Chem. Soc. 117:7452-7459.

Yantasee W, Y Lin, GE Fryxell, BJ Busche, and JC Birnbaum. 2003. "Removal of Heavy Metals from Aqueous Solution Using Novel Nanoengineered Sorbents: Self-Assembled Carbamoylphosphonic Acids on Mesoporous Silica.” Sep. Sci. Technol. 38:3809-3825. 
PNNL-16213

\section{Distribution}

No. of

Copies

OFFSITE

2 U.S. Department of Energy National Nuclear Security Agency (DOE/NA-22)

Les Pitts

NNSA/NA-22, GH-068

U.S. Department of Energy (Forrestal Bldg.) 1000 Independence Avenue, S.W.

Washington, D.C. 20585

Alan Icenhour

NNSA/NA-22, GH-068

U.S. Department of Energy (Forrestal Bldg.) 1000 Independence Avenue, S.W.

Washington, D.C. 20585

$1 \quad$ Los Alamos National Laboratory

Pat Brug

Los Alamos National Laboratory

P.O. Box 1663

Los Alamos, NM 87545
No. of

Copies

ONSITE

12 Pacific Northwest National Laboratory

S. Bonde

P8-50

R. G. Clemmer

K8-02

D. A. Dickman

$\mathrm{K} 8-46$

G. B. Dudder

K8-02

J. I. Friese

$\mathrm{P} 8-01$

J. W. Grate

K8-93

G. J. Lumetta

$\mathrm{P} 7-22$

T. C. Maiti

P7-07

M. J. O'Hara

P7-22

S. L. Petersen

P7-07

S. W. Sharpe

K8-02

J. F. Wacker

P7-07

Distr. 1 